
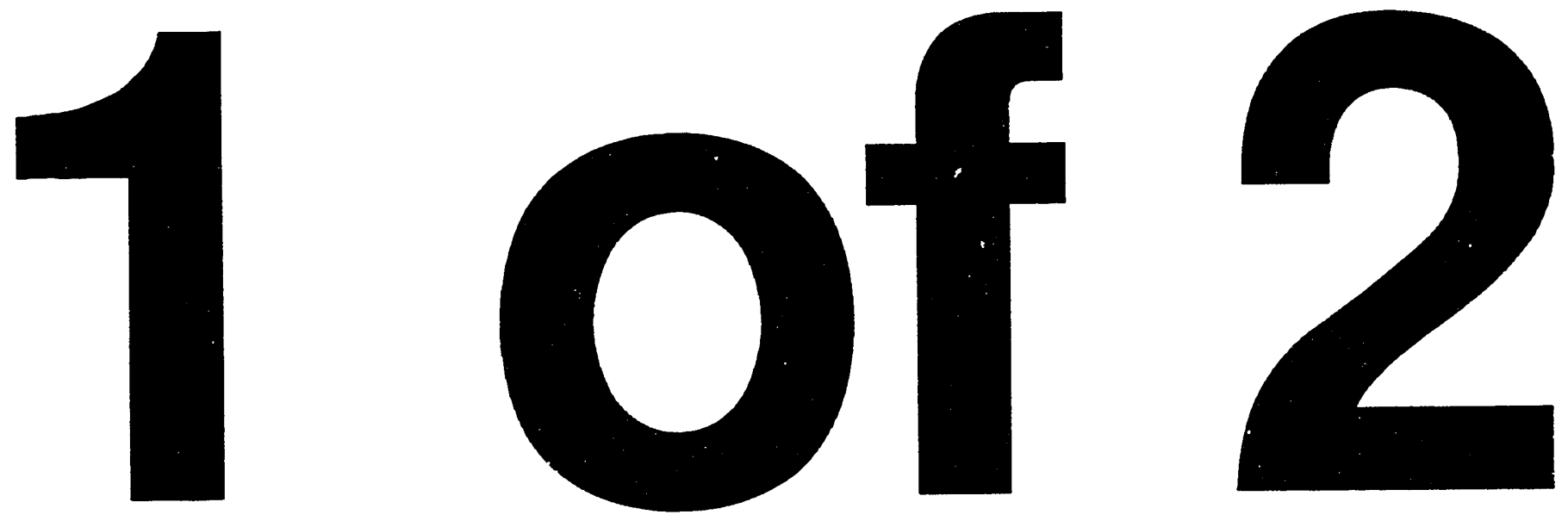
LBL-35028

\title{
STUDIES OF INTERFACES AND VAPORS WITH OPTICAL HARMONIC GENERATION
}

\author{
CHRISTOPHER SHANE MULLIN \\ Ph.D. Thesis \\ DEPARTMENT OF PHYSICS \\ University of California \\ and \\ MATERIALS SCIENCES DTVISION \\ Lawrence Berkeley Laboratory \\ University of California \\ Berkeley, CA 94720
}

DECEMBER 1993

This work was supported by the Director, Office of Energy Research, Office of Basic Energy Sciences, Materials Sciences Division, of the U.S. Department of Energy under Contract No. DE-AC03-76SF00098.

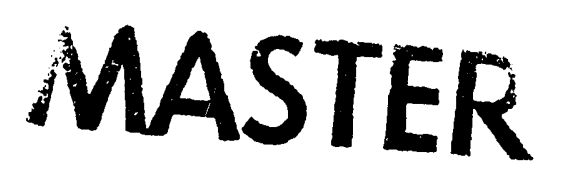




\author{
Abstract \\ Studies of Interfaces and Vapors \\ with Optical Second Harmonic Generation \\ by
}

Christopher Shane Mullin

Doctor of Philosophy in Physics

University of California at Berkeley

Professor Yuen Ron Shen, Chair

Optical Second Harmonic Generation (S. FG) has been applied to the study of soap-like molecules adsorbed to the water-air interface. By calibrating the signal from a soluble monolayer with that of an insoluble homolog, absolute measurements of the surface density could be obtained and related to the bulk concientration and surface tension. We could then demonstrate that the soluble surfactant forms a single monolayer at the interface. Furthermore, it deviates significantly from the ideal case in that its activity coefficients are far from 1 , yet those coefficients remain constant over a broad range of surface pressures. We present evidence of a first-order phase transition taking place during the adsorption of this soluble monolayer. We consider the effects of . ne non-ideal behavior and the phase transition on the microscopic model of adsorption, and formulate an alternative to the Langmuir picture of adsorption which is just as simple, yet it can more easily allow for non-ideal behavior.

The second half of this thesis considers the problem of SHG in bulk metal vapors. The symmetry of the vapor forbids SHG, yet it has been observed. We consider several models whereby the symmetry of the vapor is broken by the presence of the laser and compare their predictions to new observations we have made using a few-picosecond laser pulse. The two-lobed output beam profile 
shows that it is the vapor-plus-beam combination whose symmetry is important. The dependence on vapor pressure demonstrates the coherent nature of the radiation, while the dependence on buffer gas pressure hints at a change of the symmetry in time. The time-dependence is measured directly with a preliminary pump-probe measurement. The magnitude and intensity dependence of the signal are also measured. All but one of the models are eliminated by this comparison. The remaining model, involving ionization of the vapor, subsequent charge separation, and the generation of a macroscopic electric field, is treated in more detail and used to make $F$ edictions of future results.

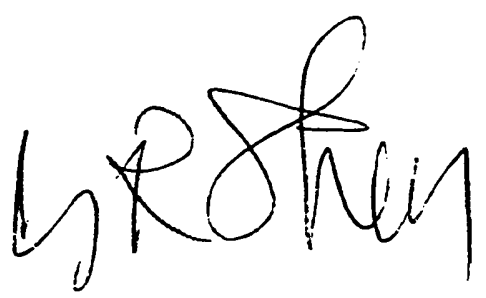




\section{Acknowledgments}

Before graduate school, before college, and even before my interest in physics, there was my family. Their love and support is the rock on which my education was built, so before all else, I want to thank them.

Second, I would like to thank Professor Yuen Ron Shen, who has dedicated many hours to my education. He has made me the physicist I am today. His training will be with me always.

I am indebted to Philippe Guyot-Sionnest, who got me through my first experiment in the Shen group. His driving willpower and damn-the-torpedoes experimental methods will always inspire me. Viola Vogel also deserves special mention for all that she taught me about Langmuir films. Her excitement about physics and sheer pleasure in doing science is all too rare in laboratories today.

I am grateful to all the members of the Shen group for their friendship and support in my many years here. They are, after all, one of the main reasons I joined the group. Rich Superfine, Marla Feller, Xu-Dong Xiao, Lorenzo Marucci, and Dieter Wilk were always there to lend a hand, lend equipment, or even lend an argument about some puzzling physics problem. Doseok Kim has been invaluable in finishing the vapor experiment and wrestling with the infamous YLF laser.

I want to thank Leo Falicov and Jeff Bokor for their valuable advice on this dissertation. With their help, it became a work of which I am indeed proud. I also want to thank my teachers all along the way - Jon Barber, Isaac Abella, Sumita Nandi, and Mark Woodworth figure highly among them.

I gladly thank the members of the UC Ballroom Dance Club for giving me a decent social life here at Berkeley. All the guys in Troop 19 can also be credited 
with reminding me that there is life outside of physics. Without such diversions, I would surely not have made it.

Special thanks go to Judy Lemire for her friendship and support, especially when I was ill. I do not know why she put up with me, but I am glad that she did.

I am especially thankful for Sarah Gaffen for getting me through the final stretch. Whether dancing, signing, or commiserating, her grin always helped me go on. When it came to the final crunch, she was there to help edit the final draft of this dissertation, turning it from a pain into a pleasure to read. I could never thank her enough. 


\section{Chapter 1: Introduction and Background}

I. Historical Perspective ....................................................................................................1

II. Framework of Optical SHG Theory....................................................................

A. Generation of a nonlinear polarization ..................................................4

B. Radiation of the nonlinear polarization..................................................5

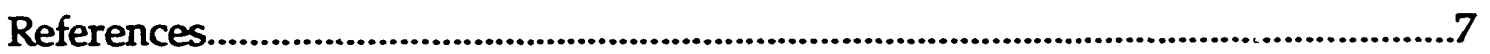

Chapter 2: Studies of Soluble Surfactants at the Air/Water Interface

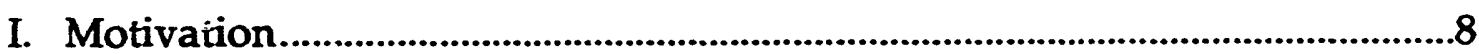

II. Theory of Stirface Adsorption ............................................................................9

A. In equilibrium ...........................................................................................10

B. Dynamics of Adsorption.........................................................................12

C. Effects of a phase transition........................................................................17

III. Sample Preparation and Experimental Arrangement....................................20

IV. Experimental Results ............................................................................................21

A. Surface Structure ...............................................................................................21

B. The Thermodynamic Equilibrium........................................................24

C. Phase Transition Evidence.............................................................................28

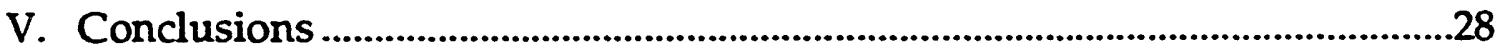

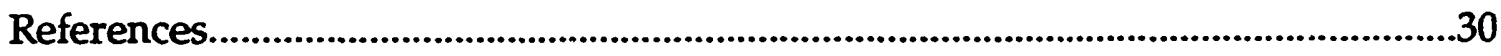

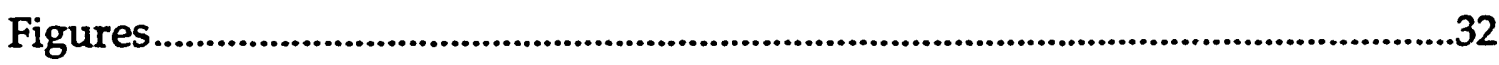

Chapter 3: Mechanisms for SHG in an Isotropic Vapor

I. Historical Background..................................................................................................43

II. Coherent Transients of Nonlinear Interactions.................................................47

III. Radiation of a Second-Harmonic Coherence ..................................................51

A. Fluorescence and superradiance ...............................................................52 


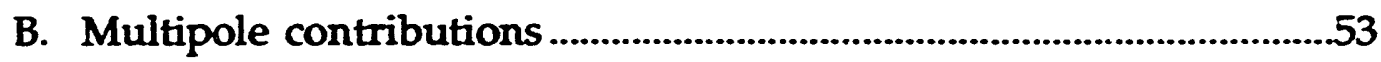

C. Collisional mechanism............................................................................57

D. Free electron nonlinearity ....................................................................59

E. Amplification due to $c(5)$........................................................................60

F. Electric Field due to Ionization.............................................................61

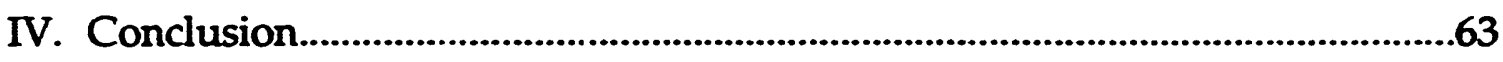

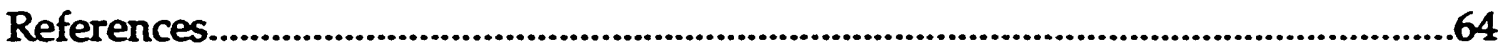

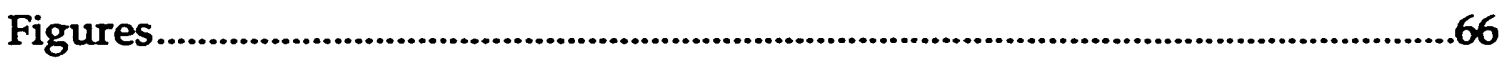

\section{Chapter 4: Results from the SHG in Vapor Experiment}

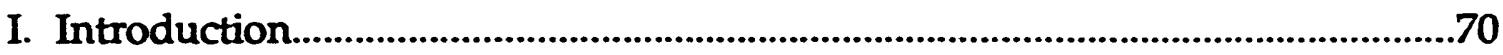

II. Evolution of a model dc Electric Field............................................................70

A. Ponderamotive potential........................................................................

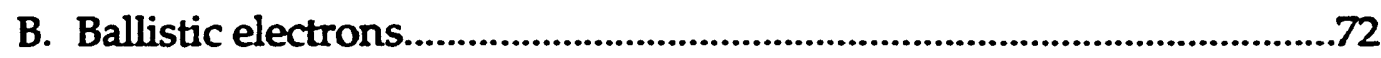

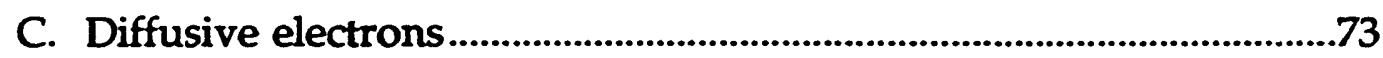

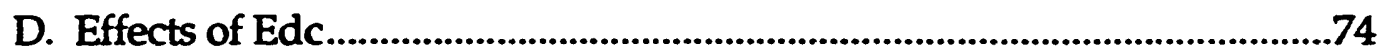

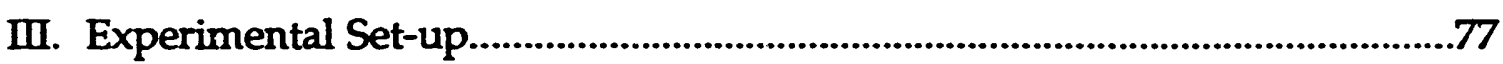

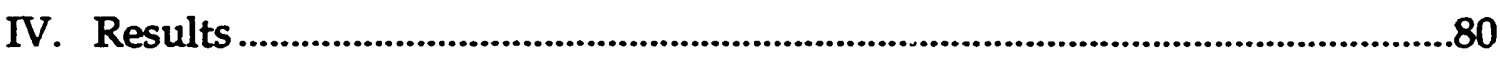

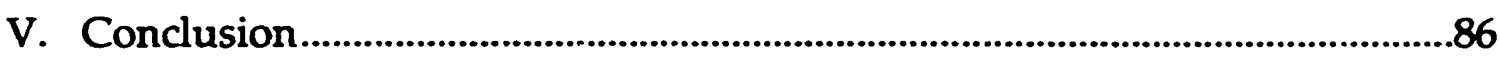

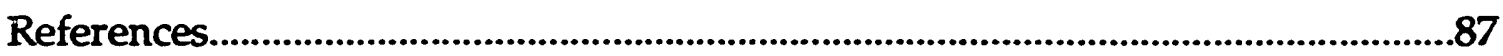

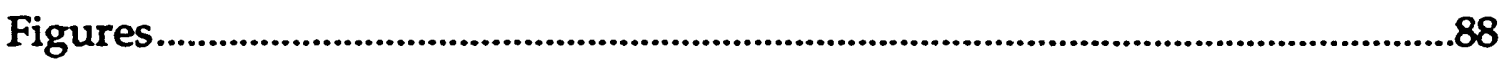

Appendix: Uncertainty in Light Measurement

I. Ways to measure light.....................................................................................18

A. Photon counting .......................................................................................109

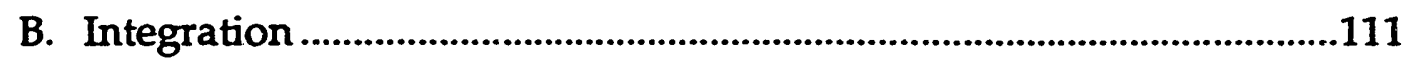

II. Uncertainty in light measurement ..................................................................112

A. Photon counting.......................................................................................112 
B. Integration ...................................................................................................113

III. Reference arms.............................................................................................115

A. Averaging over many pulses...................................................................115

B. Shot-to-shot normalization ......................................................................116

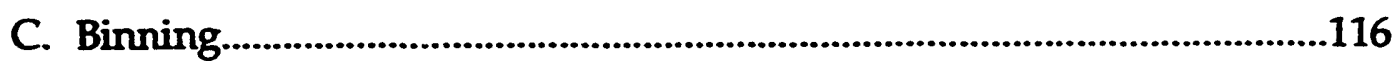

D. Checking a reference arm.........................................................................117

Figures..................................................................................................................119 


\section{Chapter 1 - Introduction and Background}

\section{Historical Perspective}

Optical Second Harmonic Generation (SHG) was first applied to the study of interfaces in 1981., ${ }^{1,2}$ Since 1981, SHG has found a respectable niche in surface science and interface studies. ${ }^{3}$ Many surface probes are "surfaceselective" only because their in-going or out-going radiation has a limited penetration depth. In contrast, SHG relies on the broken symmetry near the interface for its surface specificity, and thus it probes only the region that can be properly called the interface. Most surface science too's rely on the absorption or emission of massive particles, and are thus limited to operation in a vacuum chamber. ${ }^{4}$ SHG makes use only of light, and can be used to study any interface accessible to light, including liquid interfaces and buried interfaces.

These additional interfaces are important in many fields of physics and chemistry. Electrodes in an electrochemical cell were among the first studied with SHG. ${ }^{1}$ In another case, SHG was used to study phase transitions in monolayers of pentadecanoic acid floating on the water surface. ${ }^{5}$ Retinal molecules have also been detected in a membrane, which can be thought of as a "water-water" interface. ${ }^{6}$ SHG and the closely related process of sumfrequency generation also have advantages as spectroscopic tools. Tuning their frequencies to electronic or vibrational resonances allows them to selectively probe specific molecular species at the interface. Co-adsorbed systems that have been studied include liquid crystals on chemically 
modified surfaces. ${ }^{7}$

Two types of information are typically gained from SHG experiments: population and orientation. Population is determined through the strength of the signal, and orientation through the relative strengths of different polarization components of the signal.

However, SHG does have some limitations. If both the orientation and population of interfacial molecules are changing, it may be difficult to separate their effects on the SHG signal. Such a difficulty is not uncommon in complex interfaces. SHG is a second-order effect with signal strengths typically measured in photons or fractions of photons per laser shot. Such signal levels require several seconds or minutes to determine accurately, and thus may limit the type and time resolution of a given experiment. In Appendix A, I analyze the uncertainties in low-level light measurement and discuss methods of optimizing the efficiency of each experiment. The weak response also limits the molecules that can be studied effectively to those displaying adequate nonlinearity. This includes molecules with delocalized electronic states, as exist in phenyl groups, or where delocalized electrons are affected, as in molecular adsorption onto a metal surface. Finally, SHG requires the high intensities only found with a laser, which is often a costly and difficult tool to maintain. The intensity used in an experiment is often limited by the damage that the laser can inflict on the interface.

These difficulties, if properly navigated, can provide a tool with rich applications. Ron Shen and his collaborators have pioneered many applications of surface SHG. ${ }^{8,9}$ The first half of this thesis discusses studies of amphiphilic molecules at the air-water interface, where SHG was used to determine the structure, density, and thermodynamics of slightly soluble 
mclecules. Attempts at studying the adsorption dynamics of the molecules were largely unsuccessiul due to the iow time resolution of SHG.

The second half of this thesis concerns the problem of observed SHG in the bulk of alkali or alkali-earth vapors. Previous experiments in the field have generally relied on nanosecond lasers tuned to a two-photon resonance of the vapor (s to $\mathrm{s}, \mathrm{p}$ or $\mathrm{d}$ states), although a few have used picosecond nonresonant pulses of much higher intensity. Since the bulk symmetry forbids SHG in an unperturbed vapor, the laser itself must be breaking the symmetry of the vapor. We probed this problem with a pump-probe technique using picosecond pulses tuned to a two-photon resonance. One pulse breaks the symmetry of the medium, and a second, delayed pulse probes the broken symmetry.

The remainder of this chapter is an introduction to the theory of second-harmonic generation. Chapter 2 describes our experiments on adsorption at the water interface. Chapter 3 delves into the many processes that could generate second-harmonic light in a vapor, while Chapter 4 summarizes the results obtained in that experiment.

\section{Framework of Optical SHG Theory}

Since this thesis includes the use of SHG in several disparate problems, I will confine this chapter to the general theory of the generation of nonlinear polarization in materials and the subsequent radiation as SHG. ${ }^{10}$ The theoretical background associated with each individual problem I will leave to the theory sections of the individual chapters. In the following treatment, I will take a molecular view of the materials generating the second harmonic. Since the response at optical frequencies is dominated by the electronic 
response, I will define "molecule" as that unit to which an electron is confined. In the atomic vapor considered at the end of this thesis, that unit is a single atom. In the composite molecule used to study surface adsorption, we found that the response is dominated by the delocalized electrons in the head group of the molecules, so the unit can be thought of as simply the head group of the molecule. Weak interactions with other molecules will be considered insofar as they affect the polarizability of the primary molecule.

\section{A. Generation of a nonlinear polarization}

A molecule experiencing an electric field will respond by becoming polarized. Since the polarization is dominated by the electronic response, it can be described by the evolution of the electronic state. For simplicity, we consider only the independent electron model, so that only one-electron wave functions must be considered. Electronic excitation energies are generally much larger than the thermal energies at room temperature, so the initial equilibrium state of the electron is simply its ground state $\psi_{\mathrm{g}}$. An electric field with frequency $\omega$ will then mix the ground state with all the excited states of the electron to varying degrees, depending on the strength of the dipole matrix element between the states, and the detuning between $\hbar \omega$ and the excitation energy. Quadrupole (and higher) moment excitations can be described using the full expression of the Hamiltonian of the electromagnetic field.

The polarization moments of the molecule are then found by evaluating the expectation value of the corresponding moment operator:

$$
\begin{gathered}
\vec{P}=\langle\psi(t)| \text { e } \vec{r}|\psi(t)\rangle \\
\stackrel{\leftrightarrow}{Q}=\langle\psi(t)| \text { e } \vec{r} \vec{r}|\psi(t)\rangle \text { etc. }
\end{gathered}
$$


If the dispersion of the electronic response is negligible across the laser linewidth, then polarization response is immediate, and can be written

$$
\vec{P}(t)=\stackrel{\leftrightarrow}{\beta}(1) \cdot \vec{E}(t)+\stackrel{\leftrightarrow}{\beta}(2): \vec{E}(t) \vec{E}(t)+\ldots
$$

in which only the dipole terms are used. In frequency space, one can say that the spectral width of $\stackrel{\leftrightarrow}{\beta}$ (and hence $\vec{P}(t)$ ) is as wide as the laser linewidth, so the duration in time of the polarization is as short as that of the laser.

On the other hand, if the spectral width of $\stackrel{\leftrightarrow}{\beta}$ is narrower than the laser linewidth, then the duration in time of the response is necessarily longer than that of the laser. This fact will be critical in the analysis of a short-pulse excitation of a narrow resonance in potassium vapor.

Much of the molecule's electronic response is dictated by its symmetry. Optical SHG relies on $\stackrel{\leftrightarrow}{\beta^{(2)}}$ for generating a macroscopic electric field, and $\overleftrightarrow{\beta}$ (2) as a material property must obey the same symmetry relations as the molecule it describes. Since $\stackrel{\leftrightarrow}{\beta}(2)$ is a second-rank tensor, it must vanish for molecules with inversion symmetry, such as the isolated potassium atoms used in the bulk vapor experiment described in Chapter 4. However, even a centrosymmetric molecule may acquire a significant $\stackrel{\leftrightarrow}{\beta}(2)$ if its environment is asymmetric, a phenomenon known as microscopic symmetry-breaking. Examples include a potassium atom in an electric field or collision, and any centrosymmetric molecule adsorbed to an interface.

\section{B. Radiation of the nonlinear polarization}

Once the molecules have become polarized, they radiate in a totally linear fashion, even though their polarization was a consequence of a nonlinear interaction. However, the radiation from this polarization is quite unintuitive. In linear optics, the field due to the polarization is a small 
perturbation on the incident field. In SHG, it is the only field present at the SHG frequency. This unique situation gives rise to the problems of phase matching, $\mathrm{N}^{2}$ dependence of the radiated intensity, $\mathrm{N}$ dependence of the radiated power, etc.

To determine the macroscop $c$ polarization of a medium, one must sum the polarizations of the individual molecules:

$$
P(t)=\Sigma p(t)=\Sigma(\stackrel{\leftrightarrow}{\beta}(2): E(t) E(t))=(\Sigma \stackrel{\leftrightarrow}{\beta}(2)): E(t) E(t)=\overleftrightarrow{\chi}^{(2)}: \vec{E} \vec{E}
$$

The macroscopic polarizability is then

$$
\overleftrightarrow{\chi}(2)=\Sigma \stackrel{\leftrightarrow}{\beta}(2)=N \ll \stackrel{\leftrightarrow}{\beta}(2) \text { 》 }
$$

where the «" brackets denote an average over the molecular orientations. In any centrosymmetric bulk medium, this average will necessarily be zero. At any interface, it may be non-zero, giving SHG its surface specificity. Furthermore, it reveals that SHG is specific to only the region of material whose electronic potentials are influenced by the surface. Because of its surface specificity, SHG remains an unequaled tool in the surface scientists' arsenal. 


\section{References}

1. C. K. Chen, Phys. Rev. Lett. 46 : 1010 (1981).

2. C. K. Chen, A. R. B. de Castro and Y. R. Shen, Phys. Rev. Lett. $46: 145$ (1981).

3. Y. R. Shen, Nature 337 : 519 (1989)

4. A. Zangwill, Physics at Surfaces (Cambridge University Press, Cambridge, 1988).

5. T. Rasing, Y. R. Shen, M. W. Kim and S. Grubb, Phys. Rev. Lett. $55: 2903$ (1985).

6. T. Rasing, J. Huang, A. Lewis and Y. R. Shen, Phys. Rev. A $40: 1684$ (1989).

7. J. Y. Huang, R. Superfine and Y. R. Shen, Phys. Rev. A 42 (6): 3660 (1990).

8. Y. R. Shen, Ann. Rev. Mater. Sci. 16 : 69 (1986).

9. Y. R. Shen, submitted to Surface Science : (1993).

10. Y. R. Shen, The Principles of Nonlinear Optics (John Wiley \& Sons, New York, 1984). 


\section{Chapter 2 - Studies of Soluble Surfactants at the Air/Water Interface}

\section{Motivation}

Soluble surface-active molecules enjoy widespread use in today's industrial world. From common household items surh as soap and paint to high-technology products that require special surface treatments, these amphiphilic molecules are required to produce specific properties at liquidair, liquid-liquid, and liquid-solid interfaces. ${ }^{1}$ Yet the details of the molecules' actions and interactions near the surface remain unknown because of the paucity of tools that can be used to study these interfaces in detail. The specific problem of adsorption of amphiphilic molecules to the air/water interface is of great interest because of its wide applicability. $2,3,4,5$

The molecules are also interesting from a physics standpoint because of the unique environment in which they are situated. Insoluble amphiphilic molecules will be trapped at the interface in an essentially two-dimensional space forming Langmuir films. The large amount of work done on these films attests to their continuing fascination to researchers. Soluble amphiphilic molecules undergo transfer between the surface and bulk of the solution. Less is known about soluble surfactants because their surface density cannot be directly controlled as it can in the case of insoluble molecules. ${ }^{6}$ Models for these interfaces are often borrowed from the more intensely studied vacuum-solid interface, but the obvious physical differences that make up a fluid interface leave such analogies in doubt. 
To understand the adsorption as well as the nature of the adsorbed molecular layer, it is important to know the adsorption isotherm and the isotherm of surface pressure vs. area per adsorbed molecule $(\pi-\mathrm{A})$. While the bulk concentration of the adsorbates and the surface pressure can be easily measured, the surface density of the excess adsorbate molecules and the activity coefficients in the bulk and at the interface are not readily measurable Various techniques have been developed to probe thie surface density directly. Among them, the microtome $e^{7}$ and the radio tracer techniques ${ }^{8}$ have been most successful. However, neither one has enough spatial resolution to differentiate the signal from a surface monolayer and the signal from the subsurface layer. Furthermore, they cannot provide information about the polar orientation of the adsorbed molecules.

Recently it has been demonstrated that optical second-harmonic generation (SHG) is an effective and versatile probe for studies of molecular adsorbates at air/liquid interfaces ${ }^{9}$. It allows a direct measure of the surface density of molecules as well as providing information about their polar orientation. The technique can be used to study adsorption of soluble molecules from a solution to the air/liquid surface ${ }^{10,11,12}$. This chapter addresses four problems relating to the adsorption of soluble surfactants: the structure of the adsorbed layer ${ }^{13}$, its equilibrium relationship with bulk concentration and surface tension ${ }^{14}$, its approach to that equilibrium, and the effects of a phase transition on the layer's dynamics and equilibrium.

\section{Theory of Surface Adsorption}

In this section, I detail the theoretical relationships between microscopic parameters and the macroscopic, measurable quantities we 
determine from experiment. The first subsection considers an equilibrium between the surface and bulk parameters, and the second considers the approach to that equilibrium. An important part of these treatments is the presence of non-trivial interactions between solute molecules. The first subsection combines the interactions into the activity coefficients $f_{i}$ and $f_{b}$, while the second uses a microscopic picture of the interactions because it affects the dynamic adsorption. The last subsection examines the effect of that a first-order phase transition would have on both the dynamic and equilibrium adsorption.

\section{A. In equilibrium}

In considering a liquid system of a solute and a solvent in equilibrium, the chemical potentials of the solute in the bulk and at the air/water interface must be equal and can be written as 4

$$
\begin{gathered}
\mu=\mu_{\text {bulk }}=\mu_{b}^{0}+k T \ln \alpha_{b} \\
\mu=\mu_{\text {interface }}+\pi A^{\infty}=\mu_{i}^{0}+\pi A^{\infty}+k T \ln \alpha_{i}
\end{gathered}
$$

where $\mu_{\text {bulk }}$ and $\mu_{\text {interface }}$ are the internal chemical potentials of the solute in the bulk volume and at the interface, respectively, with $\mu_{\mathrm{b}}^{0}$ and $\mu_{\mathrm{i}}^{0}$ being the corresponding reference potentials independent of the solute densities. The activities of the solute in the bulk and at the interface are denoted by $\alpha_{b}$ and $\alpha_{i}$. One often writes $\alpha_{i}=f_{i} X_{i}$ and $\alpha_{b}=f_{b} X_{b}$ with $X_{i}$ and $X_{b}$ referring to the surface concentration (in occupied area fraction) and bulk concentration (mole fraction) of the solute, respectively, and $f_{i}$ and $f_{b}$ the corresponding activity coefficients. The surface pressure $\pi$ is defined as the change of surface tension due to the presence of the solute molecules, and $A^{\infty}$ is the surface area occupied by a solute molecule. Equations (1) and (2) lead to 


$$
\pi=-\frac{k T}{A^{\infty}} \ln \frac{\alpha_{i}}{\alpha_{b}}-\frac{1}{A^{\infty}} \Delta \mu^{0},
$$

with $\Delta \mu^{0}=\mu_{i}^{0}-\mu_{b}^{0}$.

The Gibbs' equation, derived from the second law of thermodynamics relates the variation in surface pressure to the variation of the chemical potential $\partial \mu_{\mathrm{k}}$ of the adsorbed solute molecules ${ }^{15}$ :

$$
\left.\partial \pi\right|_{T}=\left.n_{\mathbf{i}} \partial \mu\right|_{T}
$$

where $n_{i}=X_{i} / A^{\infty}$ is the surface density of solute molecules. With the help of Equation (1), we can write

$$
\left(\frac{\partial \pi}{\partial \ln \alpha_{b}}\right)_{T}=k T n_{i} .
$$

Combining equations ( 3 ) and (5) by eliminating $\pi$ yields

$$
\frac{1}{A^{\infty}}\left(1-\frac{\partial \ln \alpha_{i}}{\partial \ln \alpha_{b}}\right)+\left(-\ln \frac{\alpha_{i}}{\alpha_{b}}-\frac{\Delta \mu^{o}}{k T}\right) \frac{\partial\left(1 / A^{\infty}\right)}{\partial \ln \alpha_{b}}=n_{i}
$$

This is a general expression for the adsorption isotherm, $X_{i}$ versus $X_{b}$, knowing that $\alpha_{i}=f_{i} X_{i}$ and $\alpha_{b}=f_{b} X_{b}$. Unfortunately, the activity coefficients $f_{i}$ and $f_{b}$, as functions of $X_{i}$ and $X_{b}$, respectively, and also $A^{\infty}$ are not known a priori. Only with some assumptions can Equation (6) be simplified.

We can assume, for example, a surface site model, i. e. the actual surface area occupied by a solute molecule $A^{\infty}$ is constant and independent of the surface coverage. This gives

$$
n_{i} A^{\infty}=1-\frac{\partial \ln \alpha_{i}}{\partial \ln \alpha_{b}}
$$

If in a certain density regime, $\mathrm{f}_{\mathbf{i}}$ can be regarded as a constant independent of the bulk concentration, Equation (7) is further simplified to

$$
X_{i}=1-\frac{\alpha_{b}}{X_{i}} \frac{\partial X_{i}}{\partial \alpha_{b}}
$$

which can be integrated to yield 


$$
x_{i}=\frac{\alpha_{b}}{\alpha_{1 / 2}+\alpha_{b}}
$$

where $\alpha_{1 / 2}$ is the solute bulk activity leading to the half coverage, $X_{i}\left(\alpha_{1 / 2}\right)=$ $1 / 2$, of the surface by the solute molecules. This is in the form of the well known Langmuir adsorption isotherm.

Elimination of $\alpha_{b}$ between Equations (3) and (9) gives the $\pi-A$ (or $\pi-X_{i}$ ) isotherm:

$$
\pi=-\frac{k T}{A^{\infty}}\left\{\ln \left(1-X_{i}\right)+\ln \frac{f_{i}}{\alpha_{1 / 2}}+\frac{\Delta \mu 0}{k T}\right\}
$$

For $\pi$ versus $\alpha_{b}$, we have

$$
\pi=\frac{k T}{A^{\infty}}\left\{\ln \left(1+\frac{\alpha_{b}}{\alpha_{1 / 2}}\right)-\ln \frac{f_{i}}{\alpha_{1 / 2}}-\frac{\Delta \mu^{0}}{k T}\right\} .
$$

\section{B. Dynamics of Adsorption}

The initial conditions of the adsorption process consist of a bulk liquid with a constant concentration $X_{b}$ of surfactant filling one half space $(z<0)$, and air filling the other half space $(z>0)$. We neglect any exchange of molecules with the air. The equilibrium condition is identical, except an excess of surfactant molecules now resides at the interface. To proceed from the initial condition to the equilibrium condition, two processes must occur: molecules in the bulk must adsorb to the interface, and the molecules within the bulk must redistribute themselves to replace the molecules now adsorbed to the interface. If one process dominates the approach to equilibrium, the approach is called barrier-limited or diffusion-limited adsorption, respectively.

Adsorption models are constrained by ideal equilibrium relations between $\pi, n_{i}$ and $X_{b}$ derived from the thermodynamics above in equations (9)-(11). A consistent model must predict the form of these equilibrium 
relations in the ideal case of noninteracting molecules. The ideal case refers to $f_{i}=f_{b}=1$ and $A^{\infty}$ remaining a constant.

The presence of non-ideal interactions can be easily seen as deviations from equation $(10)^{16}$. Since optical SHG can measure $n_{i}$ without reference to $X_{b}$, it can directly observe such deviations. These deviations should be accounted for in the adsorption model. One of the simplest models to describe barrier-limited adsorption ${ }^{17}$ is due to Langmuir and is commonly used to describe adsorption of gases to the metal-vacuum interface. The only interaction acknowledged between adsorbing molecules is that a molecule adsorbed at a site blocks other molecules from adsorbing to that site. It is unable to cover cases where $\pi\left(X_{i}\right)$ deviates from the ideal.

With these assumptions, the net adsorption rate can then be written as:

$$
\left(\frac{d X_{i}}{d t}\right)_{\text {adsorption }}=k_{1} x_{b}\left(1-X_{i}\right)
$$

where $k_{1}$ is a rate constant that includes both an attempt frequency and a success rate.

The Langmuir model further assumes that a molecule adsorbed to the interface desorbs with a probability independent of the surfactant concentration, so the net desorption rate is written:

$$
\left(\frac{\mathrm{dX}_{\mathrm{i}}}{\mathrm{dt}}\right)_{\text {desorption }}=-\mathrm{k}_{2} \mathrm{X}_{\mathrm{i}} \text {. }
$$

Langmuir desorption can be thought of classically as an escape from a potential well whose depth is independent of surfactant concentration.

If $X_{b}$ remains constant, then these equations may be easily solved for $X_{i}(t):$

$$
X_{i}(t)=N_{e q}\left(1-e^{-k t}\right)
$$

where 


$$
\mathrm{N}_{\mathrm{eq}}=\frac{\mathrm{X}_{\mathrm{b}}}{\left(a+\mathrm{X}_{\mathrm{b}}\right)}, \mathrm{k}=\left(\mathrm{k}_{1} \mathrm{X}_{\mathrm{b}}+\mathrm{k}_{2}\right) \text { and } a=\frac{\mathrm{k}_{2}}{\mathrm{k}_{1}} \text {. }
$$

$N_{\text {eq }}$ denotes the equilibrium surface concentration, $X_{i}(\infty)$, which was referred to as simply $X_{i}$ in the first subsection. $k$ characterizes the rate of approach to equilibrium and $a$ characterizes the amount of adsorption in equilibrium. Thus measuring the adsorption curves for different concentrations will yield $k_{1}$ separately from $k_{2}$.

Interactions between the adsorbed molecules or different interactions between adsorbed and adsorbing molecules would change the adsorption and desorption rate equations given above. There are several ways to account for the molecular interactions. We can consider the Langmuir process above as the first terms in a polynomial expansion in $n_{i}$ of the adsorption rate, so using higher order terms could give a more correct description of the processes occurring at the surface. With the quadratic term,

$$
\left(\frac{d X_{i}}{d t}\right)=k_{1} X_{b}\left(1-X_{i}\right)-k_{2} X_{i}+k_{3} x_{i}^{2}
$$

the form of the adsorption can be solved analytically as

$$
\frac{X_{i}}{N_{e q}}=\frac{\beta_{1}(1-e q t)}{\beta_{2}-\beta_{1} e q t}
$$

where

$$
\begin{gathered}
q=\sqrt{\left(k_{1} x_{b}+k_{2}\right)^{2}-4 k_{1} k_{3} x_{b}}, \\
\beta_{1}=\frac{k_{1} x_{b}+k_{2}+q}{2 k_{3}} \text {, and } \\
\beta_{2}=\frac{k_{1} x_{b}+k_{2}+q}{2 k_{3}},
\end{gathered}
$$

A qualitative check for such a higher order term is to plot the measured values of $d X_{i} / d t$ as a function of $X_{i}$ to see if it is better fit by a parabola than a straight line. 
While attempting to fit problematic dynamic adsorption data, we formulated a new model of the fluid interface. Experimental difficulties prevent us from publishing our data, but we present the new model here as an interesting alternative to the Langmuir model. This new model is just as simple as the Langmuir model, but its different assumptions highlight the differences between fluid interfaces and solid interfaces. We did not publish the model nor the results, but I include the model here as one of my most original contributions to this thesis.

To formulate a new model, we could change the assumptions of the Langmuir model; there are three major ones: adsorbing molecules are "blocked" by adsorbed molecules; the success rate, $\mathrm{k}_{1}$, of the adsorbing molecules remains constant; and the success rate, $k_{2}$, of desorbing molecules remains constant. We propose to modify two of the assumptions: let the success frequency of the desorbing molecules depend on the surface pressure $^{18}$ :

$$
\left(\frac{\mathrm{dX}_{\mathrm{i}}}{\mathrm{dt}}\right)_{\text {desorption }}=-\mathrm{k}_{2}^{\prime} \lambda_{\mathrm{i}} \exp \left(\frac{\pi \mathrm{A}^{\infty}}{\mathrm{kT}}\right)
$$

and let adsorbed molecules not block adsorbing molecules:

$$
\left(\frac{\mathrm{dX}_{\mathrm{i}}}{\mathrm{dt}}\right)_{\text {adsorption }}=\mathrm{k}_{1}^{\prime} \mathrm{X}_{\mathrm{b}}
$$

We can test our model with the case of ideal molecules. To be consistent, the model must arrive at the thermodynamically derived relations (9) - (11). $\pi$ for ideal molecules is given by equation (10) with

$$
\ln \left(\frac{\mathrm{fi}}{\alpha 1 / 2}\right)+\frac{\Delta \mu^{0}}{\mathrm{kT}}=0
$$

so that

$$
\pi=-\frac{k T}{A^{\infty}} \ln \left(1-X_{i}\right) .
$$


Using this relation, the new model's net adsorption rate is given by

$$
\frac{\dot{u} X_{i}}{d t}=k_{1}^{\prime} X_{b}-\frac{k_{2}^{\prime} X_{i}}{\left(1-X_{i}\right)} \text {. }
$$

In equilibrium, $\frac{d X_{i}}{d t}=0$, so we recover the Langmuir adsorption isotherm, Equation (9). Thus the new model is consistent with the thermodynamic description of the interface in the case of an ideal monolayer.

Although our model and the Langmuir model predict identical equilibrium relationships for ideal surfactants, the dynamic approach to equilibrium will be different. The new model predicts a nonlinear adsorption equation which must be solved numerically. A comparison of the two families of adsorption curves is shown in Figure 1.

The two changes in the rate equations above imply two differences in the microscopic picture of the interface: in desorption, the depth of the potential well seen by the surfactant depends on the surface tension, and in adsorption, molecules at or near the water surface are mobile enough not to block adsorbing molecules. Both changes point out clear differences between the vacuum-solid interface so well-described by Langmuir kinetics, and the solution-solution surface interface considered here.

The first change stems from the fact that the molecule is not attracted to the surface by a constant force, but because it can replace solvent molecules at the surface which are in a relatively higher energy state. Thus the surfactant molecules see an effective potential well at the solvent surface. Reducing the surface tension changes the depth of the potential well, and so should increase the success rate for molecules to desorb. When a surfactant leaves the interface, the rest of the surface closes up the hole it leaves behind. This is plausible for the fluid water surface, but would be impossible on a solid interface. In the Langmuir picture, the hole is filled with nearby bulk 
molecules, so the energy cost is that of creating clean water surface. Therefore the potential well depth is independent of the surface tension. Because of this major distinction between the Langmuir model and our new one, I will call our model a "variable-depth model".

The fluidity of the surface also accounts for the non-blocking effect of the adsorbates, since without specific adsorption sites, there may be little barrier to small lateral movements of the surface molecules.

One great advantage of the variable-depth model is that it easily accounts for adsorbate interactions. Those interactions are reflected in the deviation of $\pi\left(X_{i}\right)$ from the ideal. The appearance of $\pi$ in the desorption rate equation allows empirical values of $\pi$ to be inserted directly and the equations solved numerically. While it is a large simplifying assumption to say that this is the only effect of interactions on adsorption, it is an effect that can at least be computed.

\section{Effects of a phase transition}

First-order phase transitions have often been observed in insoluble monolayers. The simplest equations that result in such a transition postulate two distinct phases at the interface with different limiting areas, $A_{1}^{\infty}$ and $A_{2}^{\infty}$ and different reference potentials, $\mu_{1}^{0}$ and $\mu_{2}^{0}$. The latter is equivalent to assuming two different, constant activity coefficients, so in the equations below I will assume that the activity coefficients are equal to one. The chemical potential is givei. by

$$
\mu_{i}\left(X_{i}\right)=\mu_{i}^{0}+\pi A_{i}^{\infty}+k T \ln \left(1-X_{i}\right)
$$

The equilibrium state will be determined by minimizing the Helmholtz free energy of the system with respect to interchange of particles 
between the two phases. At low density, only the phase with the lower $\mu_{i}^{0}$ will exist, while at high density, only the phase with lower $A_{i}^{\infty}$ will exist. The two phases will coexist only in a regime where

$$
\mu_{1}\left(X_{1}\right)=\mu_{2}\left(X_{2}\right)=\mu_{t}
$$

where $\mu_{t}$ emphasizes the fact that the chemical potential is constant throughout the transition, as are $X_{1}$ and $X_{2}$. What changes are the areas, $A_{i}$, taken up by the two phases, subject to the constraint

$$
\begin{aligned}
N_{\text {ave }} A_{\text {tot }} & =\frac{X_{1}}{A_{1}^{\infty}} A_{1}+\frac{X_{2}}{A_{2}^{\infty}} A_{2} \\
A_{\text {tot }} & =A_{1}+A_{2},
\end{aligned}
$$

where $N_{\text {ave }}$ is the average density of molecules on the surface and $A_{\text {tot }}$ is the total area of the surface. Since $\pi$ is solely a function of $X_{i}$, it is constant during the transition, and the pressures exerted by each phase must be equal:

$$
\frac{k T}{A_{1}^{\infty}} \ln \left(1-X_{1}\right)=\frac{k T}{A_{2}^{\infty}} \ln \left(1-X_{2}\right) .
$$

Equations (25) and (28) can be combined into a determining equation for $\mathrm{X}_{2}$ :

$$
\begin{gathered}
\ln \left(\frac{\left(1-X_{2}\right)^{R}-1}{\left(1-X_{2}\right)}\right)-\ln \left(X_{2}\right)+\frac{\mu_{1}^{0}-\mu_{2}^{0}}{k T}=0 \\
\text { where } R=\frac{A_{1}^{\infty}}{A_{2}^{\infty}} .
\end{gathered}
$$

Experimental evidence for such transitions in insoluble monolayers exploits the different properties of the two phases. They have different densities, resulting in different ellipsometric or SHG signals, or solubilities with tracer dyes, as used in fluorescence microscopy. ${ }^{19}$ If a laser beam is used to probe an inhomogeneous surface, its signal wili fluctuate if the size of the inhomogeneities is comparable to the laser spot size, and if the inhomogeneities move around. 
In a soluble monolayer, a phase transition would have a number of interesting effects. However, it would be difficult to detect. The clear plateau in the $\pi-\mathrm{N}$ curve visible in insoluble monolayers would appear as a kink in the $\pi-\ln (c)$ curve. The slope of $\pi-\ln (c)$, according to Gibbs' equation, would be $k T N_{\text {gas }}$ below the transition and $k T N_{\text {liquid }}$ above the transition. Such a kink would be difficult to distinguish by measuring $\pi$ alone and using Gibbs' equation. With SHG's ability to directly measure $N$, a flat $\pi-N$ relation is easily measurable.

In equilibrium, because the chemical potential of the surface adsorbates remains constant throughout the transition, the bulk chemical potential (and therefore $X_{b}$ ) also remains constant. Any solute molecules added to the bulk would adsorb to the surface until the entire surface was in the higher-density phase It would be nearly impossible to mix a solution whose surface was in the middle of the transition. Just changing the surface area of the solution could cause a complete surface phase transition.

In the microscopic picture of adsorption/desorption, the transition can be accounted for by using different rate constants for the two phases. However, it requires a lower desorption rate for the higher density phase due to attractive interactions between the adsorbed molecules. This counters the picture of oblate molecules pushing on each other to stand up. The effect would have to be more dramatic in the Langmuir model than in the variabledepth model to make up for the additional blocking effected by the higherdensity phase that is stipulated by the Langmuir model. 


\section{Sample Preparation and Experimental Arrangement}

The NS surfactants used in our experiment were $\left(\mathrm{C}_{n} \mathrm{H}_{(2 n+1)}\right)-\mathrm{C}_{10} \mathrm{H}_{8}$ $\mathrm{SO}_{3} \mathrm{Na}$, denoted as $\mathrm{CnNS}$. $\mathrm{C} 6 \mathrm{NS}$ is soluble in a $0.35 \mathrm{M} \mathrm{NaCl}$ solution of water. With the same salt concentration, C18NS appears as insoluble monolayers if spread on water. The C6NS solutions were prepared by first dissolving C6NS crystals in water of Milli-Q quality. The solution was then stirred and shaken in an ultrasonic bath for about 20 seconds. Afterwards, salt was added to provide excess ions $(0.35 \mathrm{M} \mathrm{NaCl})$. The accuracy of the bulk surfactant concentration was $\sim 5 \%$. The solution was filled into the trough. The surface was then swept by a movable barrier and the system was allowed to reach equilibrium, which happened within $15-30$ minutes. The surface pressure was measured with respect to that of a surfactant free salt solution with an absolute accuracy of $\pm 0.5 \mathrm{mN} / \mathrm{m}$. As a calibration for our SHG measurements, insoluble monolayers of C18NS on salt water were used. They were prepared by spreading C18NS from a 1/10 methanol/chloroform solution. The Langmuir trough and all glass vessels used in the experiment were always washed with sulfuric acid, rinsed repeatedly and cleaned thoroughly in an ultrasonic bath.

For the SHG experiment, the frequency-doubled Q-switched Nd:YAG laser beam with $30-35 \mathrm{~mJ}$ was focused to a spot of $1 \mathrm{~mm}$ in diameter on the surface with an incidence angle of $58^{\circ}$ from the surface normal. Its polarization was usually set at $45^{\circ}$ from the plane of incidence to maximize the signal-to-noise ratio. Other polarizations were also used to determine the specific elements of $\underset{\chi_{s}}{(2)}$. The SHG in reflection was split by a quartz Rochon polarizer, passed through two Corning 7-54 filters and a monochromator, and detected by photo multipliers with gated electronics. Each data point was 
obtained by accumulating signals over 3000 laser shots. To assure that there was no laser damage, we exposed a C18NS film on glass to the laser beam and found that the $\mathrm{SH}$ signal did not decay with time.

\section{Experimental Results}

\section{A. Surface Structure}

To use SHG as a probe of adsorbates, we first need to characterize its response to the adsorbate. Figure 2 depicts the $\mathrm{SH}$ response from a C18NS monolayer spread on water. The surface susceptibility $\chi_{\mathrm{pm}}^{(2)}$ is plotted against the surface density $\mathrm{N}$ of the C18NS molecules, where the subindices $\mathrm{p}$ and $\mathrm{m}$ refer to the p-polarization of the $\mathrm{SH}$ output and a linear polarization at $45^{\circ}$ from the incident plane of the fundamental input, respectively. The data show that $\chi_{\mathrm{pm}}^{(2)}$ is linearly proportional to $\mathrm{N}$ for surface pressures from 3 $\mathrm{mN} / \mathrm{m}$ up to $33 \mathrm{mN} / \mathrm{m}$, where the monolayer is close-packed with a limiting area of $A_{s}^{\infty}=1 / N_{s}^{\infty}=0.36 \mathrm{~nm}^{2}$ per molecule. A constant ratio of $\chi_{\mathrm{pm}}^{(2)}$ to $\chi_{\mathrm{sm}}^{(2)}$ in the linear region shows that the orientation of the adsorbed C18NS molecules remains unchanged from one-half to a full monolayer. This indicates that the molecular orientation remains unchanged above the surface pressure of $3 \mathrm{mN} / \mathrm{m}$. We were not able to quantify the average orientation of the molecules because in the present case, $\stackrel{\leftrightarrow}{\beta}$ of C18NS is not dominated by a single element as evidenced by $\chi_{z y y}^{(2)} / \chi_{y z y}^{(2)}=3.2 \neq 1$. We did have $\chi_{y y y}^{(2)}$ and $\chi_{y z z}^{(2)}$ negligibly small, indicating an isotropic orientational distribution in the surface plane. For the work here, however, the quantitative information about the molecular orientation is not needed. Below 1.2 molecules $/ \mathrm{nm}^{2}, \chi_{\mathrm{pm}}^{(2)}$ show large fluctuations (shown in Figure 3) and then become vanishingly small. This is presumably due to an 
orientational phase transition into a face-flat position. The above results have two important implications. First, the local field effect arising from the interaction between C18NS molecules is negligible since otherwise, $\chi^{(2)}$ would be nonlinear in $\mathrm{N}$. Second, $\chi^{(2)}$ is a linear measure of the total number of polar-oriented molecules adsorbed at the interface as denoted in Chapter 1.

Since the hydrocarbon chain on CnNS contributes negligibly to the optical second-order nonlinearity, the hyperpolarizability $\stackrel{\leftrightarrow}{\beta}$ for $\mathrm{C} 6 \mathrm{NS}$ is essentially identical to that for C18NS. If the chromophore orientation of C6NS and C18NS at the interface are also the same, then the measured $\chi^{(2)}$ from SHG can directly be used to determine the surface density of the polaroriented C6NS molecules in the interfacial layer. This is indeed the case, as confirmed experimentally. Above 1.2 molecules $/ \mathrm{nm}^{2}$, the ratio of $\chi_{\mathrm{pm}}^{(2)} / \chi_{\mathrm{sm}}^{(2)}$ for the C6NS surface layer is the same as that for the C18NS, showing that the two molecules have the same chromophore orientation. We can therefore use our C18NS measurements, where the surface density is easily measured, as a calibration of our C6NS measurements, where the density is unknown.

We have studied SHG from the air/water interface of a C6NS solution. In Figure $4 \mathrm{a}$, the measured $\chi_{\mathrm{pm}}^{(2)}$ are plotted against the bulk concentration $X_{b}$ of C6NS in the solution. It is seen that $\chi_{\mathrm{pm}}^{(2)}$ increases with $\mathrm{X}_{\mathrm{b}}$ and approaches saturation. A comparison with Figure 2 reveals that $\chi_{\mathrm{pm}}^{(2)}$ (C6NS) at saturation equals $\chi_{\mathrm{pm}}^{(2)}(\mathrm{C} 18 \mathrm{NS})$ of a close-packed monolayer, given by the dotted line in Figure 4a. This indicates that in both cases, one with soluble and the other with insoluble molecules, the interfacial layer contains the same number of polar-ordered naphthalene sulfonate molecules. It is likely that $\mathrm{t} \cdot ? \mathrm{p}$ polarordered C6NS molecules also appear at the interface as a single close-packed 
monolayer. However, the possibility still exists that the interfacial layer is composed of a partially polar-ordered C6NS surface monolayer and some polar-ordered molecules in the subsurface region.

The following experiment was carried out to reject the possibility of polar-ordering in the subsurface. A monolayer of insoluble C18NS molecules was spread on top of the C6NS solution. For low surface densities of C18NS, we should have C6NS and C18NS molecules coadsorbed at the interface. Reducing the surface area forces the soluble C6NS molecules to submerge into the water. Eventually, only the C18NS molecules would float on the surface and form a close-packed, totally polar-oriented monolayer. We found that whether the close-packed monolayer of C18NS was on salt water or on the C6NS solution, the nonlinear optical responses $\chi_{\mathrm{pm}}^{(2)}$ are the same. This indicates that beneath a polar-ordered C6NS monolayer at the surface of a C6NS solution, there should not be a subsurface layer of C6NS with partial polar-ordering.

In a separate experiment, an insoluble monolayer of eicosanol (C20$\mathrm{OH}), \mathrm{C}_{20} \mathrm{H}_{41}-\mathrm{OH}$, was spread on top of the $\mathrm{C} 6 \mathrm{NS}$ solution. The $\mathrm{OH}$ head group has a different polarity than that of the naphthalene sulfonate head group. Thus the polar-ordering of C6NS in the subsurface layer underneath a $\mathrm{C} 20-\mathrm{OH}$ monolayer, if present, could be different. Figure 5a depicts the measured surface tension $(\pi)$ versus the mean area per $\mathrm{C} 20-\mathrm{OH}$ molecule (A) for three different C6NS concentrations in the solution, $c_{b}=0 \mu \mathrm{M}, 200 \mu \mathrm{M}$ and $600 \mu \mathrm{M}$. It is seen that at low surface densities of $\mathrm{C} 20-\mathrm{OH}$, the surface tension for the three cases are very different because different numbers of C6NS molecules are coadsorbed with $\mathrm{C} 20-\mathrm{OH}$ at the interface. Upon compression to reduce the surface area, however, the curves with $c_{b} \neq 0$ 
asymptotically approach the one with $c_{b}=0$. This indicates that the adsorbed C6NS molecules can be squeezed back into the water eventually leaving only a close-packed $\mathrm{C} 20-\mathrm{OH}$ monolayer at the interface, and that $\mathrm{C} 6 \mathrm{NS}$ molecules do not form an ordered subsurface layer underneath the $\mathrm{C} 20-\mathrm{OH}$ monolayer. The process of squeezing coadsorbed soluble molecules back into solution by monolayer compression of insoluble surface molecules has also been observed with SHG by Eisenthal and coworkers. ${ }^{20}$ This conclusion is supported by the SHG results shown in Figure $5 b$, where $\chi_{\mathrm{pm}}^{(2)}$ is plotted against A for $c_{b}=0 \mu \mathrm{M}, 200 \mu \mathrm{M}$ and $600 \mu \mathrm{M}$. Although the values of $\chi_{\mathrm{pm}}^{(2)}$ are different for the $\mathrm{C} 20-\mathrm{OH}$ monolayer on water and on the $\mathrm{C} 6 \mathrm{NS}$ solution at large $\mathrm{A}$ [note that $\stackrel{\leftrightarrow}{\beta}(\mathrm{C} 6 \mathrm{NS})>\stackrel{\leftrightarrow}{\beta}(\mathrm{C} 20-\mathrm{OH})$ ], they become nearly equal towards the limiting value of $\mathrm{A}$ at which the $\mathrm{C} 20-\mathrm{OH}$ molecules form a close-packed monolayer. The result shows that as the C6NS molecules are driven back into water by compression, they do not form any partially polar-ordered subsurface layer underneath the $\mathrm{C} 20-\mathrm{OH}$ monolayer.

\section{B. The Thermodynamic Equilibrium}

It is of fundamental interest for adsorption studies to find the relations between the surface pressure $\pi$, the number of surface molecules $n_{i}$ and the bulk concentration $X_{b}$. We now examine the details of adsorbed C6NS surface films in the presence of excess ions ${ }^{8,21}$. For soluble surfactants in equilibrium, the surface density depends on the bulk concentration. We have measured SHG from the surface of a C6NS solution and obtained $\chi_{\mathrm{pm}}^{(2)}$ as a function of the C6NS bulk mole fraction $x_{b}$. Then with Figure 2 relating $\chi_{\mathrm{pm}}^{(2)}$ and $\mathrm{n}_{\mathrm{i}}$, the adsorption isotherm for C6NS can be deduced as shown by circles in Figure $4 a$. 
The surface pressure $\pi$ versus $X_{b}$ for C6NS were measured simultaneously with SHG. The results are shown as full circles in Figure $4 \mathrm{~b}$. As expected, both $\pi$ and $n_{i}$ increase with increasing bulk concentration until the critical micelle concentration ( $\mathrm{cmc}$ ) is reached. The solid lines will be discussed later.

With these two independent sets of data in Figure 4, i.e. $n_{i}$ versus $X_{b}$ and $\pi$ versus $X_{b}$, the adsorption process can now be analyzed quantitatively. We are particularly interested in testing the simplifying assumptions discussed in the theory section. Consider first the general equation, Equation (3), which we express in the form

$$
\pi=-\frac{k T}{A^{\infty}} \ln \frac{X_{i}}{X_{b}}-\Delta \pi\left(f_{i}, f_{b}, \Delta \mu 0\right)
$$

with

$$
\Delta \pi=\frac{k T}{A^{\infty}} \ln \frac{f_{i}}{f_{b}}+\frac{1}{A^{\infty}} \Delta \mu^{0} .
$$

We now assume $A^{\infty}$ constant and independent of $X_{i}$ or $n_{i}$ so that $\Delta \pi$ would depend on $X_{i}$ and $X_{b}$ only through the activity coefficients $f_{i}$ and $f_{b}$. From the maximum density of an adsorbed full monolayer of C6NS, as calibrated by a close-packed monolayer of C18NS, we found $A^{\infty}=0.36 \mathrm{~nm}^{2}$. For a given $X_{b}$, the measured $\pi$ and $X_{i}$ allow us to deduce $\Delta \pi$ from Equation (30). This was actually carried out with the data in Figure 4 and surprisingly $\Delta \pi$ was found to be $-154 \pm 0.8 \mathrm{mN} / \mathrm{m}$ independent of $X_{b}$. A negative sign for $\Delta \pi$ is expected for surfactants since their surface state is lower in energy than the bulk state. In Figure 6 , we plot the data of $\pi$ versus $X_{b}$ directly from the experiment and $\left(-\frac{k T}{A^{\infty}} \ln \frac{X_{i}}{X_{b}}+154\right) \mathrm{mN} / m$ versus $X_{b}$ calculated from the measured $X_{i}$ versus $X_{b}$. It is seen that the two sets of data coincide very well. The result here not only confirms the assumption of $\mathrm{A}^{\infty}$ being constant but also suggests that $f_{i} / f_{b}$ is independent of the bulk as well as the surface 
concentration of the solute in the range covered by our experiment. We find $f_{i} / f_{b}$ to be constant within the experimental error of $8 \%$. It is possible that both $f_{i}$ and $f_{b}$ are independent of $X_{i}$ and $X_{b}$.

Consider next the Gibbs' equation, Equation (5). If we assume a constant $f_{b}$, then Equation (5) becomes

$$
x_{b}\left(\frac{\partial \pi}{\partial X_{b}}\right)_{T}=k T n_{i}
$$

Again, this can be checked by the experimental data. A polynomial best fit of the experimental of $\pi$ versus $X_{b}$ is given in Figure $4 b$ as solid line. From the slope we can then calculate $n_{i}(\pi)$ versus $X_{b}$ from Equation (31) as given as solid curve in Figure 4a. The number of surface molecules calculated from the Gibbs equation agrees well within $\pm 5 \%$ with the measured $n_{i}$ (SHG) versus $X_{b}$ data at lower bulk mole fractions. As $X_{b}$ approaches the $\mathrm{cmc}$, the inaccuracy in the determination of the slope of $\pi$ versus $X_{b}$ is tremendous and not given in this figure. However, the slope does approach $\frac{1}{X_{b}} \frac{k T}{A^{\infty}}$ as $X_{b}$ approaches the cmc, as expected from Equation (31). The results here suggests that $f_{b}$ can indeed be regarded as constant in the range of $X_{b}$ we have covered. With $f_{i} / f_{b}$ being constant, this implies that $f_{i}$ is also constant in that range. Physically, the activity coefficients are measures of the intermolecular interaction. The bulk $f_{b}$ is independent of $X_{b}$ presumably because $X_{b}$ is very small in our case. It is surprising to see $f_{i}$ constant. This may be due to the narrow range of $X_{i}$ probed in our case so that the effect of the variation of intermolecular interaction on $f_{i}$ is not significant.

With $f_{i}$ and $f_{b}$ approximately constant, the Langmuir adsorption isotherm, Equation (9), becomes

$$
n_{i} A^{\infty}=\frac{X_{b}}{X_{1 / 2}+X_{b}} .
$$


As shown in Figure 4a, Equation (32) describes the data reasonably well with $X_{1 / 2}=\frac{40}{55} \times 10^{-6}$ as read from the graph at half the surface coverage. A Langmuir type adsorption isotherm was also found by others 22 . With Equation (32), we can express Equation (31) of $\pi$ versus $X_{b}$ in the form

$$
\pi=\frac{k T}{A^{\infty}} \ln \left(1+\frac{X_{b}}{X_{1 / 2}}\right)-\Delta \bar{\pi}
$$

with $\Delta \bar{\pi}=\frac{k T}{A^{\infty}} \ln \frac{f_{i}}{f_{b} X_{1 / 2}}+\frac{\Delta \mu^{0}}{A^{\infty}}$. For an ideal system of non-interacting adsorbates $\left(f_{i}=f_{b}=1\right)$ we can derive $X_{1 / 2}=\exp \left(\frac{\Delta \mu^{0}}{k T}\right)$ from the standard Langmuir adsorption model and have $\Delta \bar{\pi}=0$. In that case, $X_{1 / 2}$ would be a direct measure for the adsorption energy $\Delta \mu^{0}$ of the surfactant molecules (for $X_{1 / 2}=\frac{40}{55} \times 10^{-6}, \Delta \mu^{0}=-8.2 \mathrm{kcal} /$ mole for the ideal system). Generally, we $\operatorname{expect} \Delta \bar{\pi} \neq 0$. In Figure $4 b$, Equation (33) is plotted with $\Delta \bar{\pi}=0 \mathrm{mN} / \mathrm{m}$ and $\Delta \bar{\pi}=4.8 \pm 0.2 \mathrm{mN} / \mathrm{m}$. It is seen that the latter describes the data satisfactorily. This clearly indicates that our system is non-ideal, i.e. although $f_{i} / f_{b}$ is constant in the range of $X_{b}$ covered, it is different from 1. Unfortunately, without knowing $\Delta \mu^{0}$ separately, we cannot deduce the value of $f_{i} / f_{b}$ from $\Delta \bar{\pi}$. However, if we assume that the relation $X_{1 / 2}=\exp \left(\frac{\Delta \mu^{0}}{k T}\right)$ is still approximately true, then we can find $\frac{f_{i}}{f_{b}}=1.5$ from the values of $\Delta \bar{\pi}$ and $X_{1 / 2}$. If we assume $f_{b} \approx 1$ for our diluted system, the surface activity coefficient $f_{i}$ is larger than one. This is expected if the effect of surface exclusion dominates attractive interaction among the surface molecules and the result is in agreement with other experiments ${ }^{23}$.

Finally, the surface pressure/area isotherms are given in Figure 7 for the soluble C6NS molecules adsorbed to the air/water interface (o) and for the insoluble C18NS monolayer on film compression (solid line). The dotted 
curve is -alculated from Equation (33) with $\Delta \bar{\pi}=4.8 \mathrm{mN} / \mathrm{m}$. It is interesting to note that the isotherms cross.

\section{Phase Transition Evidence}

We have observed with these optical techniques that a first-order phase transition oscurs in the insoluble homolog of the family, S18NS, in that we observe fluctuations in SHG signal below an average density of $1.5 \mathrm{~mol} / \mathrm{nm}^{2}$ (Figire 3). Furthermore, we observe evidence of a first-order phase transition in the soluble homolog, C10NS. During very slow adsorption, the ellipsometry signal fluctuates below the critical density of $1.5 \mathrm{~mol} / \mathrm{nm}^{2}$ (Figure 8). These fluctuations reach intensities comparable to the signal at the critical density, and so can be interpreted as coming from regions which are the size of the laser spot, or larger, and have the critical density of molecules, even though the average density is much lower. These islands, observed during dynamic adsorption, imply a first order surface phase transition in this soluble surfactant molecule.

We would be more confident in the existence of this transition if we could observe a plateau in the $\pi$ - $\mathrm{N}$ diagram (Figure 9). Unfortunately, in this case, $\pi$ is below our experimental resolution of $0.1 \mathrm{mN} / \mathrm{m}$.

\section{Conclusions}

In this chapter, we have investigated many aspects of the adsorption of surfactant molecules to the water/air interface. We have used SHG's unique capabilities to prove that the adsorbed surfactant forms a single monolayer at the water surface. We have measured independently the surface pressure and the surface density of soluble surfactant molecules as function of the bulk concentration of these molecules in solution. The system investigated here, 
hexadecyl naphthalene sulfonate adsorbed to the air /salinated water interface, behaves as non-ideal gas in the pressure range from $2 \mathrm{mN} / \mathrm{m}$ up to surface saturation. We could show that the ratio of the surface activity coefficient to the bulk activity coefficient deviates significantly from unity. Both activity coefficients are approximately constant over the concentration range probed. This is a very interesting finding. Various models in the literature $6,8,15,23$ that describe adsorption equilibrium do not seem to apply here. The surface activity coefficient must depend only weakly on the dipoledipole or Coulomb forces among surface molecules which should increase with increasing packing density. For the bulk phase it is known that the bulk undergces a phase transition from monomers to the formation of micelles when the surface density approaches saturation with increasing bulk concentrations. Discussion, however, exists on the sharpness of this phase transition 24,25 . A constant bulk activity coefficient expresses that the aggregation number for surfactants in the bulk is approximately constant. Therefore, a gradual change of the aggregation number as direct precursor to the cmc can be excluded.

We have also seen evidence for a surface phase transition in a soluble monolayer. We have proposed a new model, based on a variable-depth potential well at the liquid surface, as an alternative to Langmuir adsorption. The variable-depth model points out some interesting differences between liquid and solid surfaces, but it clearly needs to be tested with experiments. 


\section{References}

1. D. K. Chattoraj and K. S. Birdi, "Chapter 3," in Adsorption and the Gibbs Surface Excess, (Plenum Press, New York, 1984),

2. P. Schaaf and J. Talbot, Phys. Rev. Lett. 62 (175): (1989).

3. E. M. Lee, R. K. Thomas, J. Penfold and R. C. Ward, J. Phys. Chem. 93 : 381 (1989).

4. D. H. Everett, Trans. Faraday Soc. $61: 2478$ (1965).

5. A. Y. Meyer, D. Farin and D. Avnir, J. Am. Chem. Soc. 108 : 7897 (1986).

6. A. W. Adamson, Physical Chemistry of Surfaces (Wiley, New York, 1990).

7. J. W. McBain and R. C. Swain, Proc. Roy. Soc. A 154 : 608 (1936).

8. K. Tajima, Bull. Chem. Sa. Jap. 43 : 3063 (1970).

9. V. Vogel and Y. R. Shen, Ann. Rev. Mater. Sci. 21 : 515 (1991).

10. T. Rasing, T. Stehlin, M. W. Kim, P. Valint Jr. and Y. R. Shen, J. Chem. Phys. 89 : 3386 (1988).

11. K. Bhattacharyya, A. Castro, E. V. Sitzmann and K. B. Eisenthal, J. Chem. Phys. 89 : 3376 (1988).

12. K. Bhattacharyya, E. V. Sitzmann and K. B. Eisenthal, J. Chem. Phys. 87 : 1442 (1987).

13. V. Vogel, C. S. Mullin, Y. R. Shen and M. W. Kim, Langmuir 7 (6): 1222 (1991).

14. V. Vogel, C. S. Mullin, M. W. Kim and Y. R. Shen, J. Chem. Phys. 95 (6): 4620 (1991).

15. J. T. Davies and E. K. Rideal, Interfacial Phenomena (Acad Press, New York, 1963).

16. J. F. Baret, J. Colloid. Int. Sci. 30 (1): 1 (1969). 
17. R. P. Borwankar and D. T. Wasan, Chem. Eng. Sci. 38 (10): 1637 (1983).

18. G. Glasstone, J. J. Laidler and H. Eyring, The Theory of Rate Processes (McGraw-HIll, New York, 1941).

19. C. M. Knobler, Physica 140 A : 198 (1986).

20. X. Zhao, M. C. Goh and K. B. Eisenthal, J. Phys. Chem. 94 : 2222 (1990).

21. I. N. Tang, H. R. Munkelwitz and N. Wang, J. Colloid. Int. Sci. $114: 409$ (1986).

22. P. Perea-Carpio, F. Gonzalez-Caballero, J. M. Bruque and G. Pardo, J. Colloid. Int. Sci. 95 : 513 (1983).

23. A. F. H. Ward and L. Tordai, Tıans. Faraday Soc. 42 : 408 (1946).

24. K. Motomura, S. I. Iwanaga, S. Uryu, H. Matsukiyo, M. Yamanaka and R. Matuura, Coll. Surf. 9 : 19 (1984).

25. J. N. Israelachvilli, in Proc. Int'l School of Phys. "Enrico Fermi", V. Degiorgio, Eds. (North Holland Pub. Co., New York, 1983), 


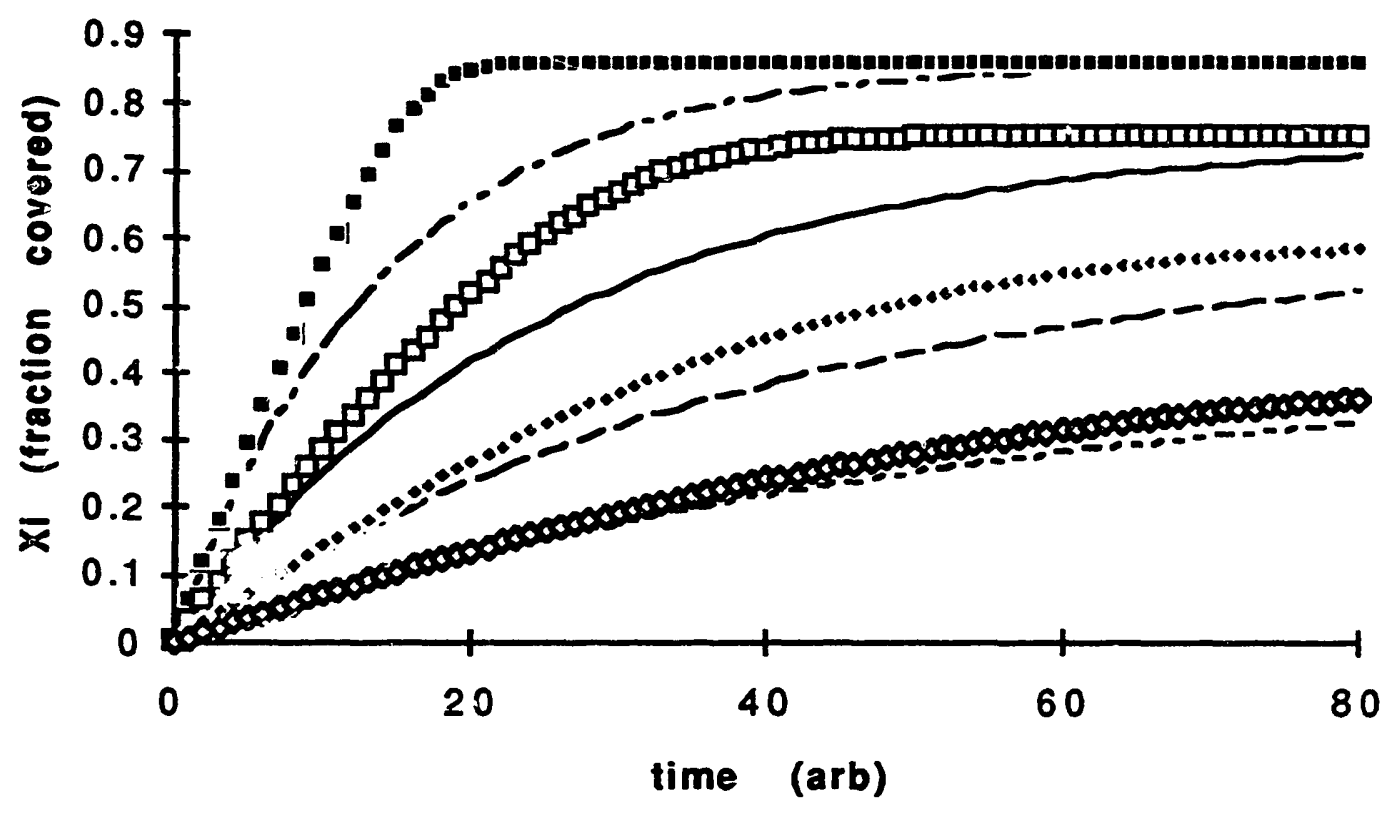

Figure 1: Comparison of Langmuir (lines) and variable-depth models (symbols) in dynamic adsorption of ideal molecules for four different surfactant concentrations. 


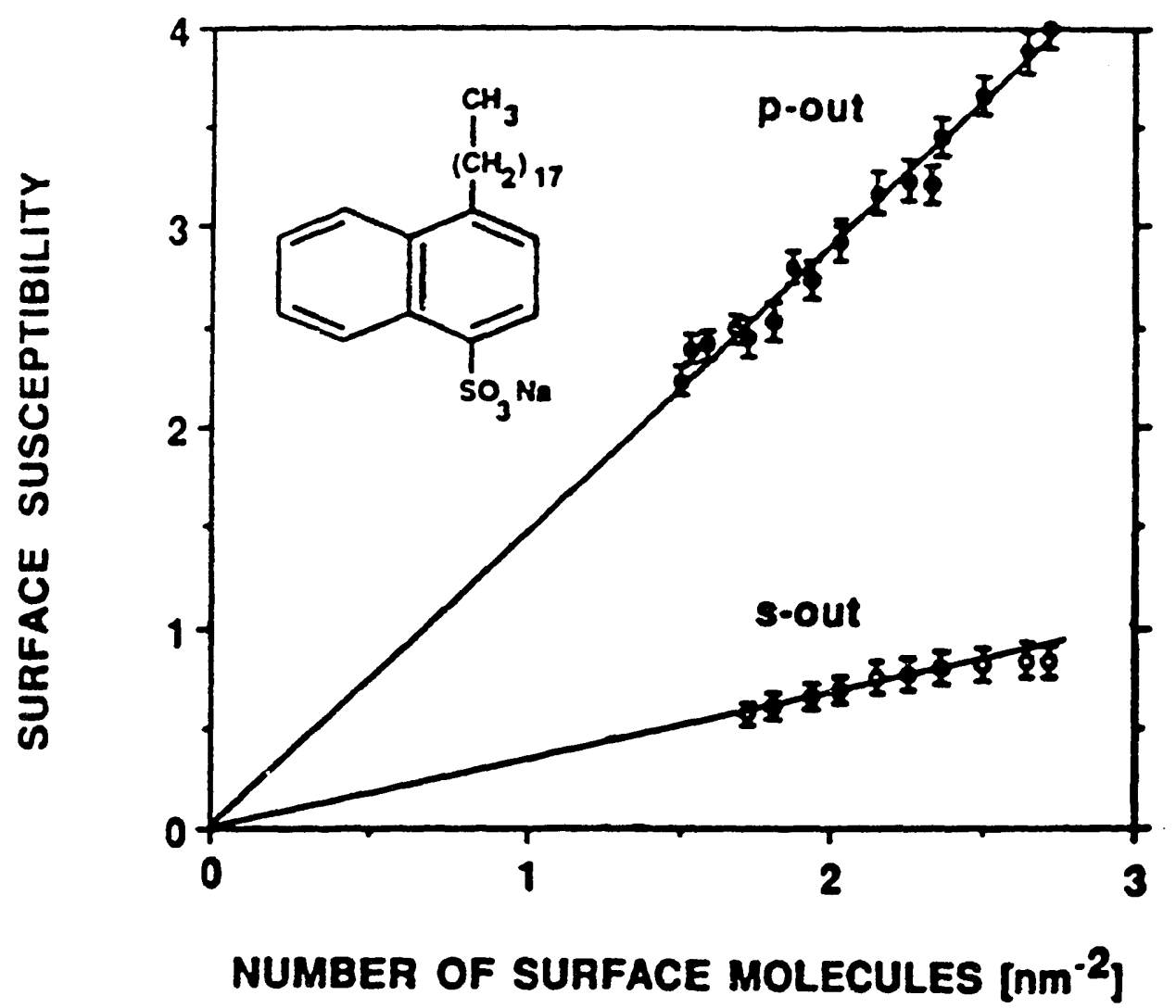

Figure 2: Second-order surface susceptibility versus the number of surface molecules for an insoluble monolayer of C18NS at the air/water interface in the presence of excess counter ions $\left(0.35 \mathrm{M} \mathrm{NaCl}, 20^{\circ} \mathrm{C}\right)$. The polarization combinations used are $\mathrm{p}$ - and s-polarization of the $\mathrm{SH}$ output and a linear polarization at $45^{\circ}$ from the incident plane of the fundamental input. 


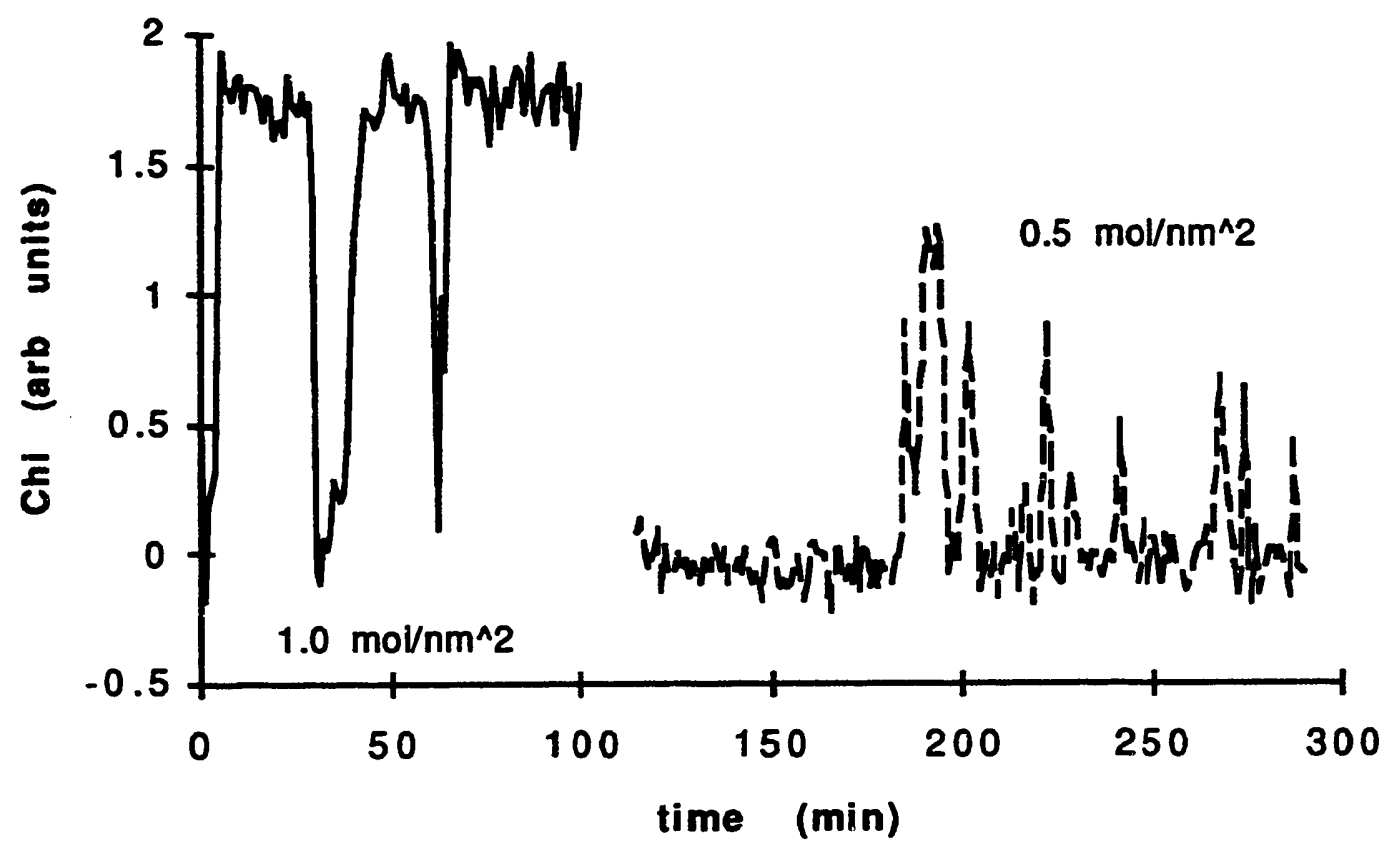

Figure 3: Optical SHG measurements as a function of time for two different averare surface densities of the insoluble C18NS molecule. The fluctuations indicate an inhomogeneous surface on the scale of the laser beam diameter. 


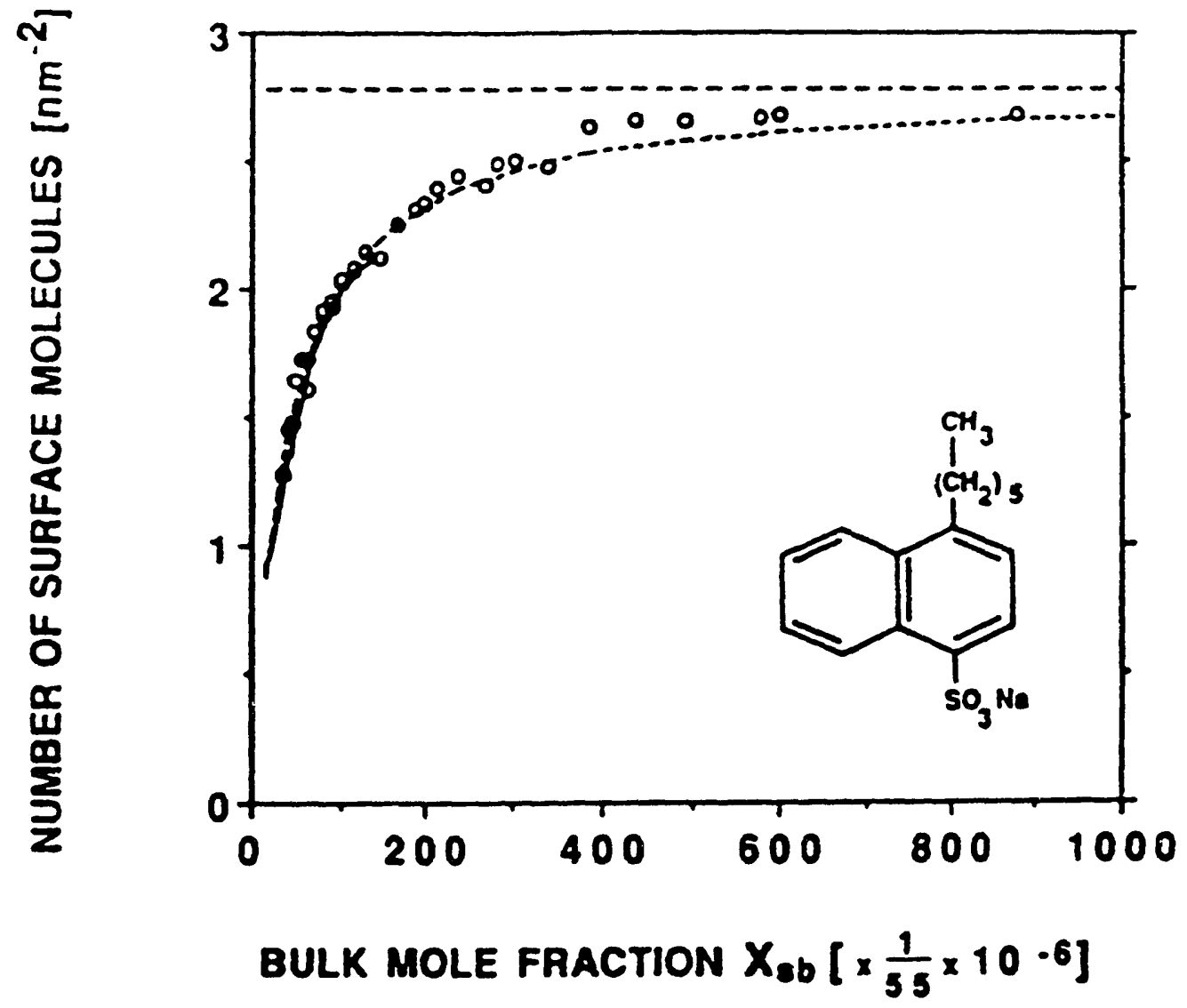

Figure 4a: Number of surface molecules versus the bulk mole fraction of the soluble $\mathrm{C} 6 \mathrm{NS}$ molecules $\left(0.35 \mathrm{M} \mathrm{NaCl}, 20^{\circ} \mathrm{C}\right)$. The lines represent the surface coverage at saturation (-- ) , the number of surface molecules calculated from the Gibbs' equation (Equation 5) (__) and the Langmuir equation (Eq. 32) (-) with $X_{1 / 2}=\frac{40}{55} \times 10^{-6}$, respectively. 


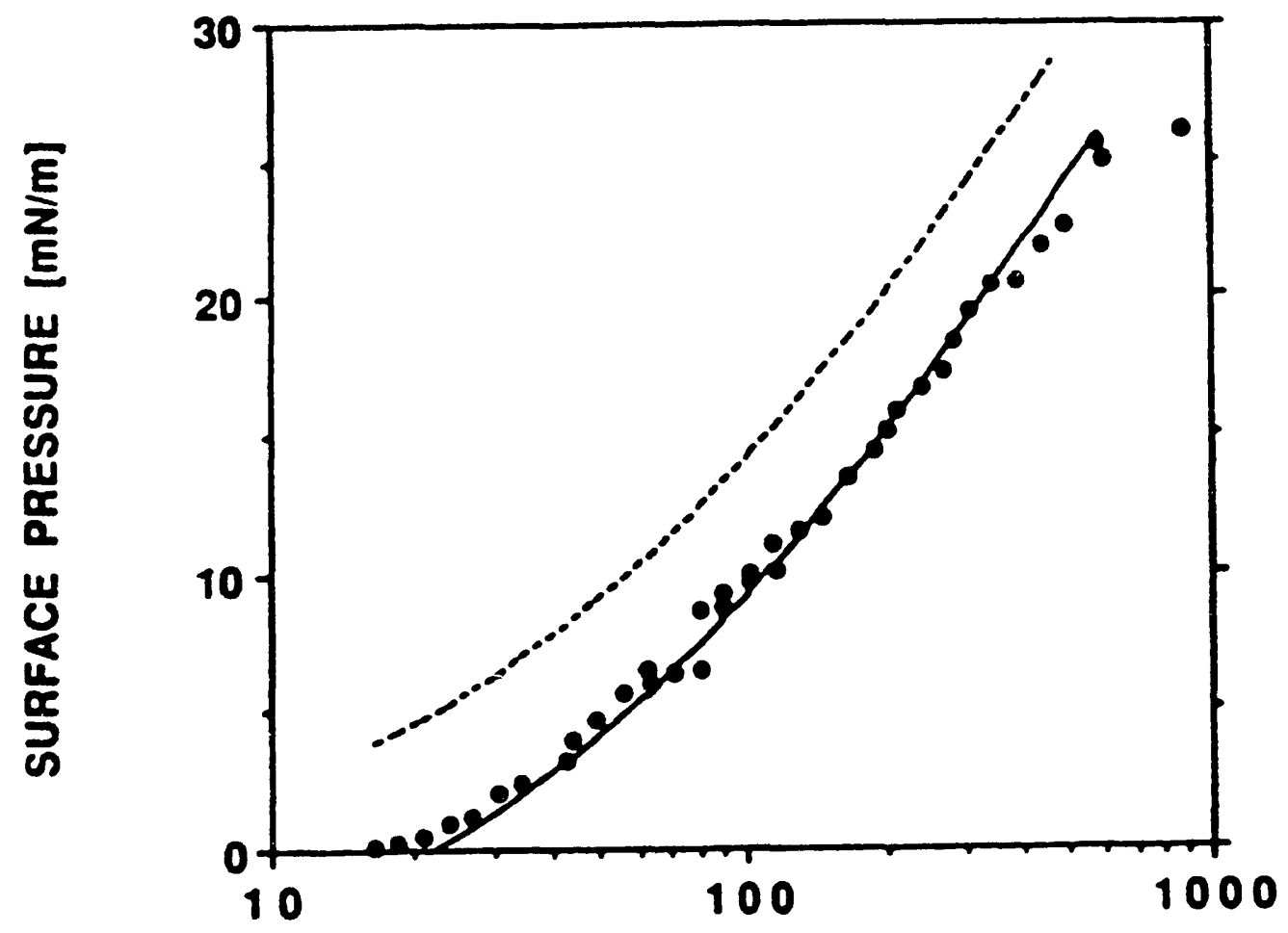

BULK MOLE FRACTION $X_{s b}\left[\times \frac{1}{55} \times 10^{-6}\right]$

Figure 4b: Surface pressure versus the bulk mole fraction of the soluble C6NS molecules $\left(0.35 \mathrm{M} \mathrm{NaCl}, 20^{\circ} \mathrm{C}\right)$. The lines are calculated from Equation (33), for an ideal system of non-interacting adsorbates with $\frac{f_{i}}{f_{b}}=$ $1, \Delta \bar{\pi}=0 \mathrm{mN} / \mathrm{m}(---)$, and for $\frac{f_{i}}{f_{b}}>1, \Delta \bar{\pi}=4.8 \mathrm{mN} / \mathrm{m}$ ( 


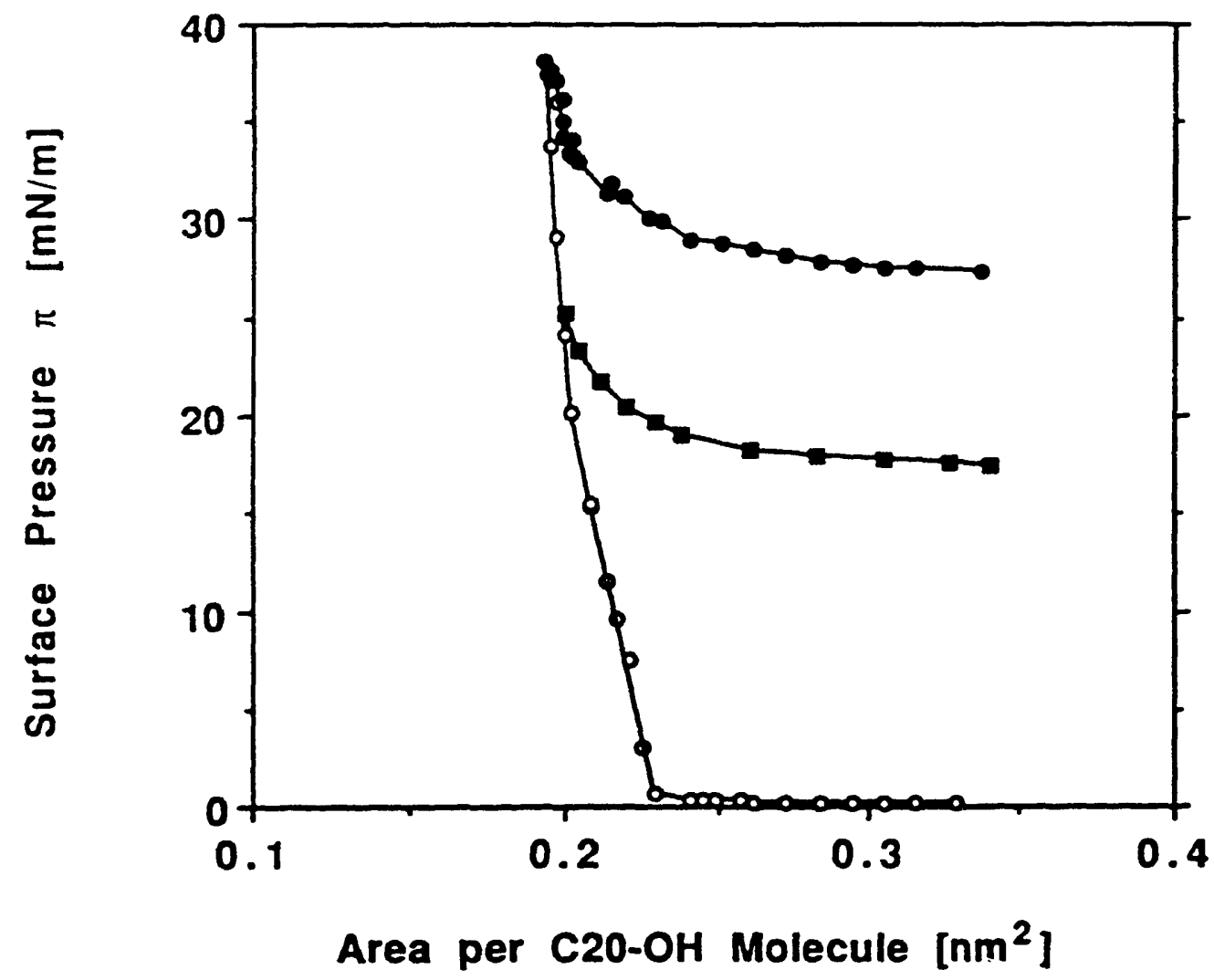

XBL $903-5416$

Figure 5a: Experimental results of surface pressure versus mean area per $\mathrm{C} 20-\mathrm{OH}$ molecule for a $\mathrm{C} 20-\mathrm{OH}$ monolayer on plain water $(0.35 \mathrm{M} \mathrm{NaCl}$ solution with $\mathrm{pH}=5.6$ at $\left.20^{\circ} \mathrm{C}\right)(\mathrm{o})$ and on solutions with $\mathrm{C} 6 \mathrm{NS}$ bulk concentrations of $200 \mu \mathrm{M}(\bullet)$ and $600 \mu \mathrm{M}(\bullet)$. 


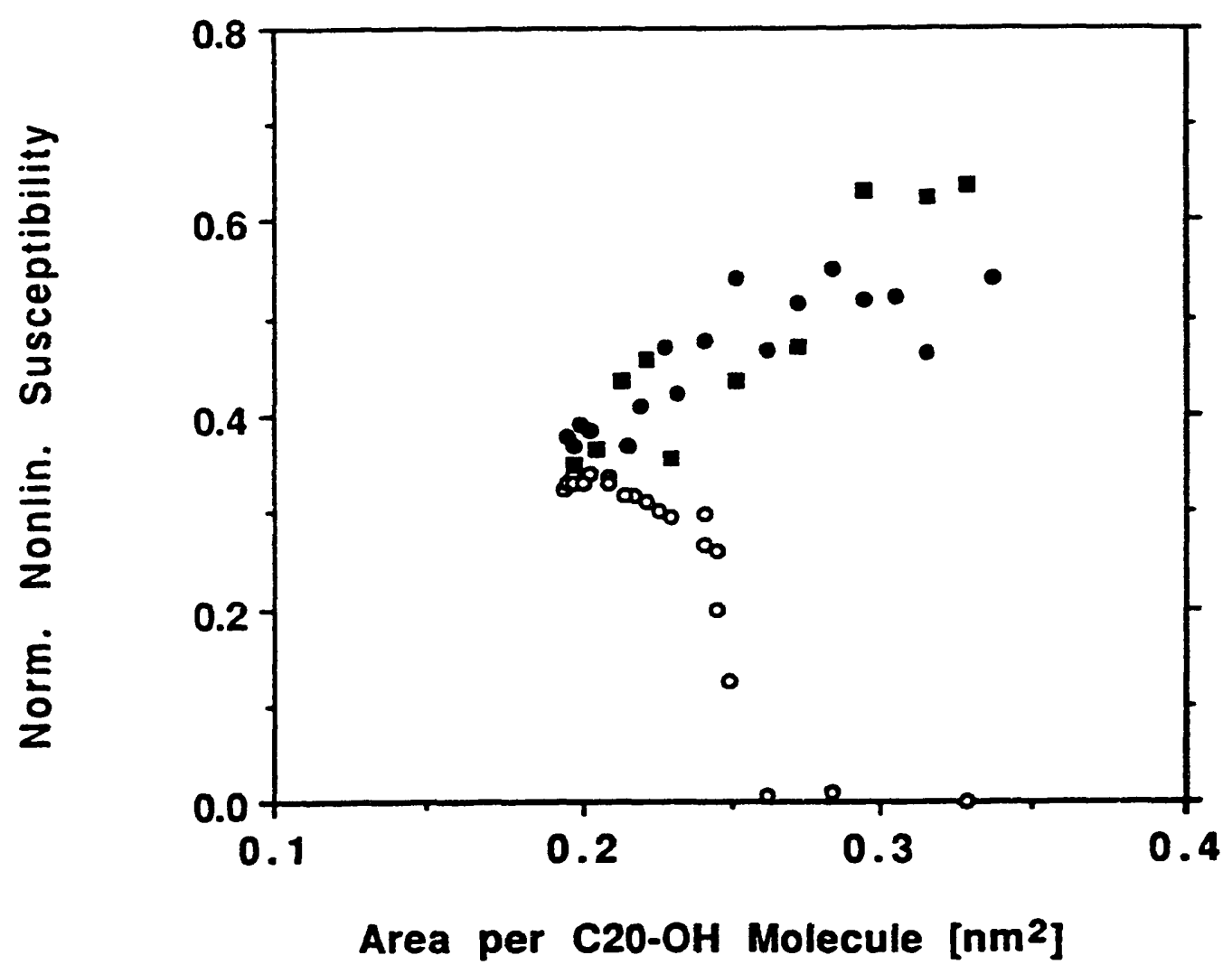

XBL 903-5417

Figure 5b: Experimental results of second-order susceptibility versus mean area per $\mathrm{C} 20-\mathrm{OH}$ molecule for a $\mathrm{C} 20-\mathrm{OH}$ monolayer as in Figure 5a: on plain water (0) and on solutions with C6NS bulk concentrations of $200 \mu \mathrm{M}(\square)$ and $600 \mu \mathrm{M}(\bullet)$. The second-order susceptibility is normalized with respect to the signal from a close-packed C6NS adsorbate layer in the absence of $\mathrm{C} 20-\mathrm{OH}$ molecules. The fluctuations in the nonlinear response from the interface of a $\mathrm{C} 20-\mathrm{OH}$ monolayer spread on a C6NS solution can presumably be attributed to phase separation of soluble and insoluble molecules in the surface monolayer. 


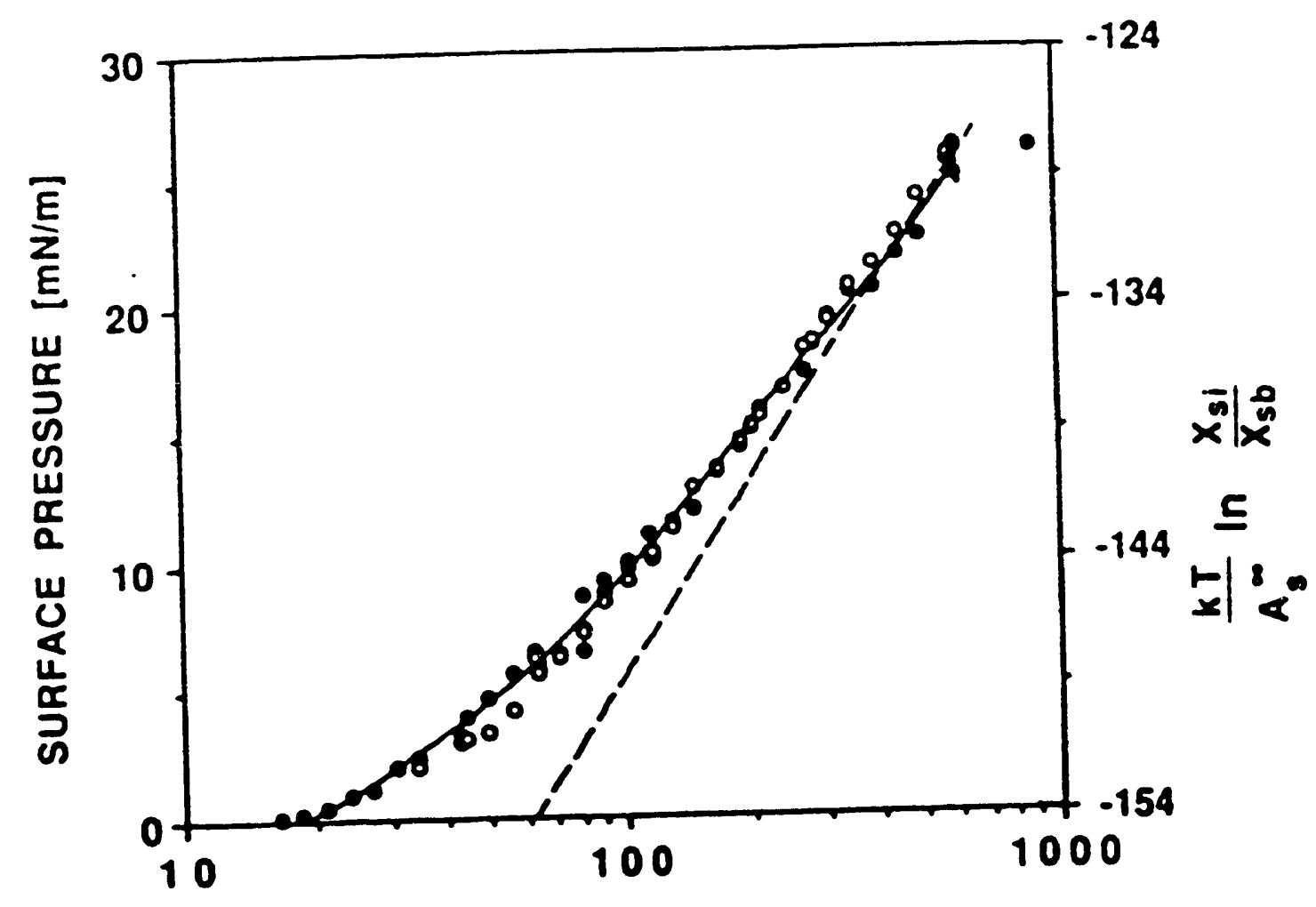

BULK MOLE FRACTION $X_{8 b}\left[\times \frac{1}{55} \times 10^{-6}\right]$

Figure 6: The surface pressure on the left $(\bullet)$ and $-\frac{k T}{A^{\infty}} \ln \frac{X_{i}}{X_{b}}(0)$ on the right ordinate as obtained from the SHG measurement versus the bulk mole fraction of C6NS molecules. The two data sets coincide very well if the ordinates are shifted by $\Delta \pi=-154 \mathrm{mN} / \mathrm{m}$, respectively. The solid line represents a polynomial fit to the surface pressure data. Its slope reveals the number of surface molecules (Gibbs' Eq. (5)) and is given in Figure $4 a$ (solid line). At surface saturation the slope approaches $1 / A^{\infty}$ with $A^{\infty}$ $=.36 \mathrm{~nm}^{2}$ given by the straight dotted line. 


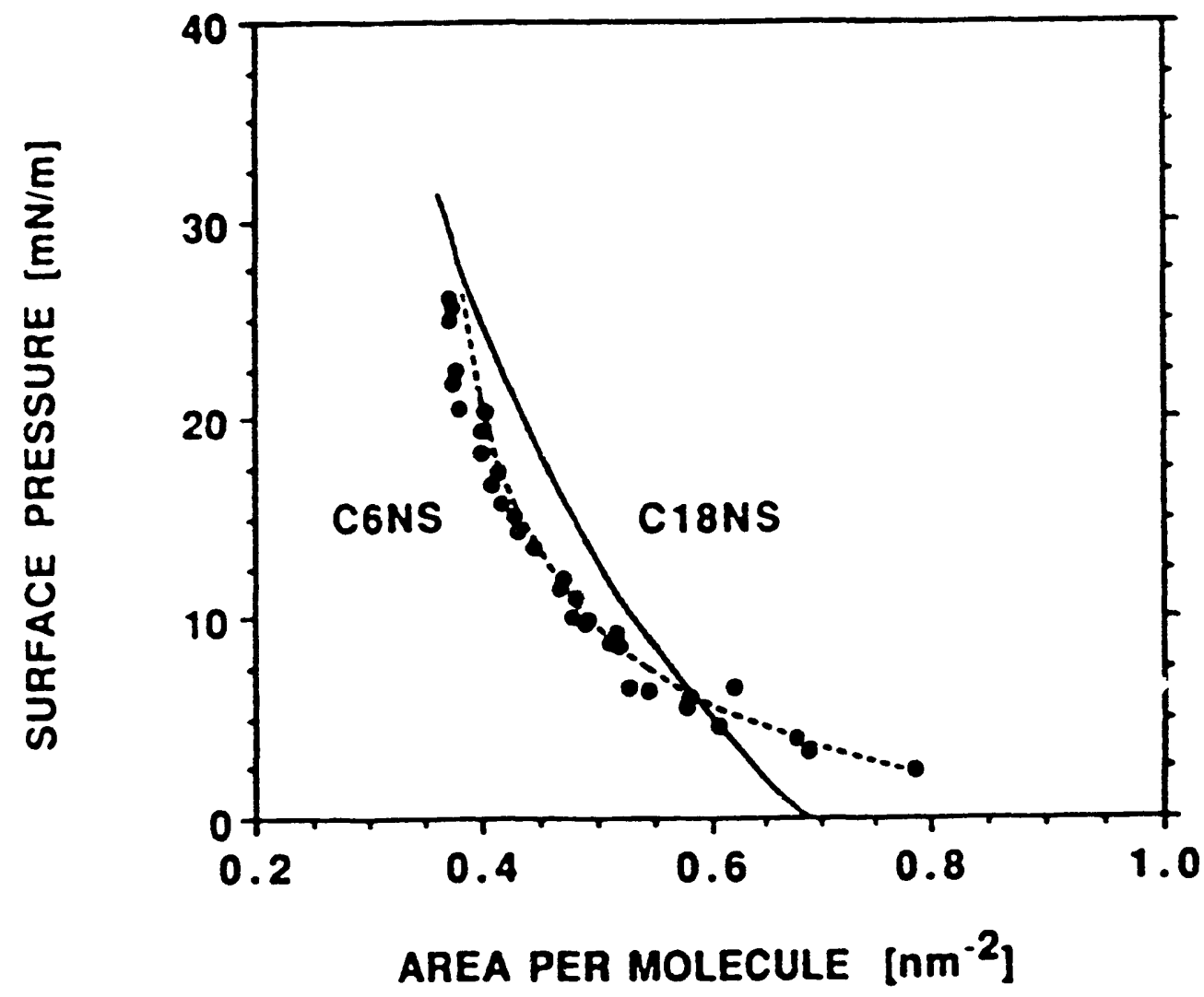

Figure 7: Surface pressure versus the mean area per molecule for the insoluble monolayer of C18NS (_) and the soluble C6NS film ( $\bullet$ ) $\left(0.35 \mathrm{M} \mathrm{NaCl}, 20^{\circ} \mathrm{C}\right)$. The former isotherm is governed on compression of the insoluble C18NS monolayer, the latter on adsorption of C6NS molecules to the air/water interface for various bulk concentrations. In the adsorption study, the area per molecule is obtained from the SHG measurement, the dotted curve is calculated from $\pi=-\frac{k T}{A^{\infty}} \ln \left(1-X_{i}\right)$ $\Delta \bar{\pi}$ with the derivation given in the text. 


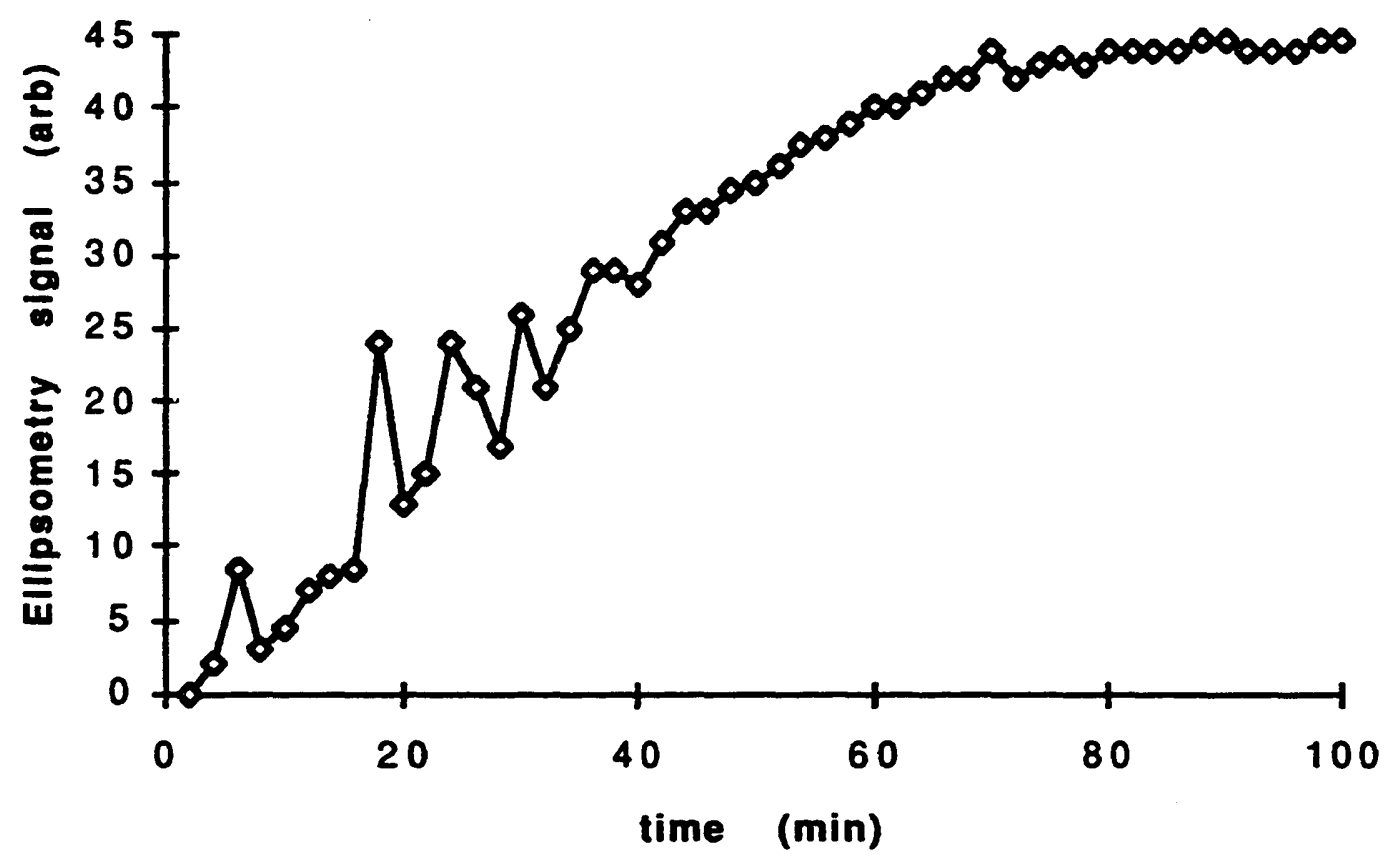

Figure 8 Fluctuations observed with ellipsometry during C6NS dynamic adsorption, indicating for the first time the presence of a phase transition in a soluble monolayer. 


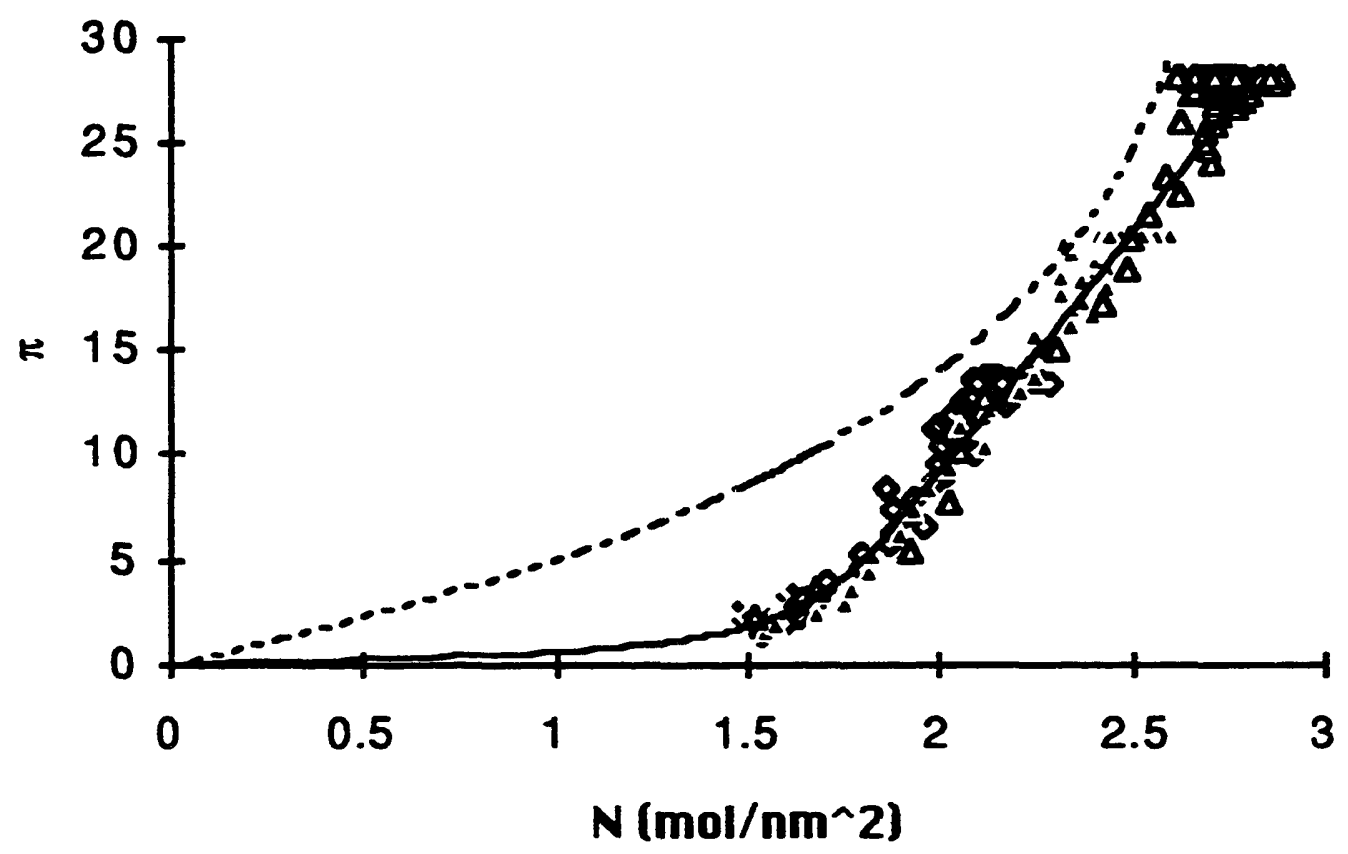

Figure $9 \pi$-N curve measured during dynamic adsorption at several concentrations: $0.6 \mu \mathrm{M}$ - filled diamonds; $1.0 \mu \mathrm{M}$ - open diamonds; 1.5 $\mu \mathrm{M}$ - filled triangles; $2.0 \mu \mathrm{M}$ - open triangles. The symbols cluster in a horizontal line at their maximum pressure because the pressure is more accurately measured than the SHG signal for any one point. The dotted curve shows the expected dependence for an ideal surfactant with the same limiting area, while the solid curve is an empirical functional fit. 


\section{Chapter 3 - Mechanisms for SHG in an Isotropic Vapor}

\section{Historical Backoround}

A strong argument for the utility of SHG as a surface probe is the fact that it is forbidden in a centrosymmetric bulk. However, there have been observations s, $^{1,3,4}$ of appreciable SHG observed in a bulk metal vapor when a laser is tuned to a second-harmonic resonance in that vapor. We sought to resolve this disparity using a new tunable amplified picosecond laser system built by our group. This chapter and the next present cur current understanding of this problem based on our new experimental results.

Three-wave mixing, of which SHG is but one example, is not allowed in isotropic vapors in the dipole approximation. ${ }^{5}$ When an external electric or magnetic field ${ }^{6}$ is used to break the symmetry, three-wave mixing has been observed and understood. When higher-multipole processes are enhanced by a noncollinear geometry, sum frequency generation has also been observed and understood. ${ }^{7}$ But in 1977, Flusberg, et al., reported difference-frequency mixing in the absence of any external field ${ }^{6}$, and soon thereafter reported second-harmonic generation at a two-photon resonance. ${ }^{3}$ His result was not understood.

Flusberg's finding sparked a series of experiments in which SHG was observed in several alkali $8,9,10,11$ and alkali-earth vapors, $12,13,14,15$ and at both one-photon (s-p) and two-photon (s-d, p-p, and s-s) resonances. ${ }^{1}$ The s-s observation was particularly important because s-s transitions have no multipole moment. Thus multipole process cannot explain the s-s 
observation.

The presence of SHG in a bulk vapor is a complex problem. The resonance enhances many nonlinear optical effects, and dipole-allowed effects of higher order can easily compete with and affect the dipole-disallowed SHG. More than fifty spectral lines have been observed and their processes identified ${ }^{16}$ when a nanosecond laser is tuned to the lowest possible twophoton transition. The processes involved include energy pooling, dimers, collision-induced effects, fluorescence, and wave-mixing. However, the most important effects are the third-order processes of four-wave mixing and threephoton ionization.

Explanations for SHG must account for symmetry-breaking on two levels: microscopically, the unperturbed atoms of the vapor are centrosymmetric, so their individual second-harmonic polarizabilities $\beta$ are zero. In other words, SHG involves a two-photon, even-parity transition up and a one-photon, odd-parity transition down. Thus, SHG cannot connect two states of definite parity, such as the levels in a spherically symmetric atom. The second symmetry-breaking is macroscopic; even if the atoms did possess a non-zero $\stackrel{\leftrightarrow}{\beta}$, the orientational average of $\stackrel{\leftrightarrow}{\beta}$ in Equation (5) of Chapter 1 will be zero in an isotropic vapor. These considerations eliminate a pure electric dipole mechanism as the explanation of vapor SHG.

In this chapter, a number of mechanisms are eliminated by comparison with the picosecond experiment's results. The remaining modei ${ }^{3,17}$ states that the ionization of the vapor leads to a macroscopic separation of charges. The resulting dc electric field breaks the microscopic symmetry of the atoms by mixing states of different parity. It also breaks the macroscopic symmetry because it is constant over a region (the beam radius) larger than a wave- 
length of light.

The ionization model was called to question because in several cases $^{1,15}$ there appeared to be no correlation between the ionization measured and the SHG observed. It was assumed that stronger ionization would create a stronger dc electric field. In Chapter 4 it is shown that the electric field saturates at the levels of ionization expected in these experiments.

The strongest of the alternative models ${ }^{1}$ involves collisions between the excited atoms and the buffer gas. The collisions mix the states of the atom and allow SHG. Evidence for their model includes a similarity between the variation of SHG efficiencies and the measured collisional cross-sections ${ }^{18}$ of an atomic species as the principle quantum number $n$ is varied. Although this model does account for microscopic symmetry-breaking, it is not clear how the macroscopic symmetry is broken. I attempted to augment this model by considering the mechanism of population gradients, but find that the resulting efficiencies are far below those that are observed.

In this chapter I will detail each proposed mechanism and show how each would be predicted to behave under different experimental conditions. These behaviors are then compared to experimental results to determine the SHG mechanism. The major characteristics that we observe in the experiment, and which the models should predict are:

- Several hundred second-harmonic photons are generated from a linearly polarized $3 \mathrm{psec}$ laser pulse focused to $400 \mu \mathrm{m}$ beam waist and $2 \times 10^{10}$ $W / \mathrm{cm}^{2}$ intensity in a $10 \mathrm{~cm}$ column of $10^{16}$ molecule $/ \mathrm{cm}^{3}$ potassium vapor.

- Second-harmonic light appears for both 4s-9d and 4s-11s two-photonresonant tunings of the laser. Strong light is generated at both the 10p and $11 \mathrm{p}$ resonances, and is thought to be due to allowed four-wave-mixing 
processes.

- With a 4s-9d tuning, light is also generated at $11 \mathrm{~s}, 10 \mathrm{~s}$, and $8 \mathrm{~d}$ resonances, with strengths slightly weaker than that of the second-harmonic.

- The SHG light is collimated, but is split into two lobes oriented along the polarization direction of the input laser beam.

- The SHG light is emitted within the time resolution of our photomultiplier tubes, which is $10 \mathrm{nsec}$.

- The SHG light is strongly intensity dependent (I6 or greater), but it quickly saturates.

- The SHG light is polarized primarily in the direction of the input polarization.

- The SHG light decreases with increasing Ar pressure faster than $1 / P$. I will examine these results in detail in Chapter 4.

Most of the previous experiments were done using nanosecond tunable dye lasers tuned into one- or two-photon atomic resonances. Several experiments have been reported using non-resonant picosecond sources, ${ }^{19,20}$ but these were done at very high intensities where perturbative calculations break down. All picosecond experiments used the ionization model to explain their results.

Our experiment bridged the gap between these two types of experiments by using amplified few-picosecond pulses tuned to two-photon resonances to test SHG in potassium vapor. Because of the resonant enhancement, the experiment used much lower intensities than the previous picosecond experiments. By splitting the pulse into two and delaying one pulse with respect to the other, we planned to determine the time evolution of whatever broken symmetry was allowing the SHG. By separating the 
symmetry-breaking from the SHG, we attempted to determine the mechanism.

In the sections below, I first re-examine the assumption of broad resonances made in Chapter 1 , which is clearly violated in the case of the narrow atomic resonances of a vapor. Then I outline the mechanisms that were considered but eliminated based on the experimental results. Finally, I describe a mechanism by which a dc electric field can allow second-harmonic radiation in the vapor and the characteristics such radiation would have assuming a radial dc electric field. The details of how the $\mathrm{dc}$ field should evolve and their experimental consequences will be covered in Chapter 4.

\section{Coherent Transients of Nonlinear Interactions}

At low pressures, the atomic resonance is narrower in frequency space than the spectral width of a picosecond laser pulse. This is the opposite of the assumption in Chapter 1 that the nonlinear polarizability of the molecules was constant in frequency space. As a result, the atomic response must be treated as a coherent transient excitation. In other words, the atomic state at time $t$ is determined by the history of the electric field and not just by the electric field at time $t$. The effective potential due to the field can be calculated from second-order time-dependent perturbation theory. The states are labeled as follows:

$\mid \mathrm{g}>: 4 \mathrm{~s}$, the ground state of room temperature atomic potassium.

Im > : intermediate states, typically $4 \mathrm{p}$, which is $4000 \mathrm{~cm}^{-1}$ away from the laser's frequency. To be exact, calculations should be summed over all intermediate states, but I drop the summing for these rough calculations.

|f , : final state, $2 \hbar \omega$ above the ground state. In our experiments we 
tuned to $9 \mathrm{~d}, 10 \mathrm{~d}, 11 \mathrm{~s}$, and $12 \mathrm{~s}$ resonances.

|r > : state with which |f > must be mixed in order to radiate as a dipole with the ground state. The most effective $\mid r>$ states are $p$-states close to the excited states, such as 10p and 11p.

These levels are diagrammed in Figure 1. Initially, all atoms are in Ig >. The population of If $>$ is then determined through perturbation with the electric potential $\mathrm{q} \overrightarrow{\mathbf{E}} \cdot \vec{x}$. To first order,

$$
c_{f}(1)(t)=\frac{-i}{\hbar} \int_{0}^{t} q E x_{f g} \exp \left[i\left(\omega_{g f}-\omega\right) t^{\prime}\right] d t
$$

where $\omega$ is the laser frequency, and we have assumed a square pulse approximation for the laser field. For two states of the same parity, $\mathbf{x}_{\mathrm{fg}}=0$, so we proceed to second order:

$$
\begin{aligned}
& c_{f}(2)(t)=\left(\frac{-i}{\hbar}\right)^{2}(q E)^{2} x_{f m} x_{m g} \int_{0}^{t} \exp \left[i\left(\omega_{f m}-\omega\right) t\right] d t \\
& x \int_{0}^{t^{\prime}} \exp \left[i\left(\omega_{m g}-\omega\right) t^{\prime}\right] d t^{\prime \prime} \\
& =\frac{(q E)^{2} x_{f m} x_{m g}}{\hbar^{2}\left(\omega_{m g}-\omega\right)}\left(\frac{\exp \left(i\left(\omega_{g f}-2 \omega\right) t\right)-1}{\left(\omega_{g f}-2 \omega\right)}-\frac{\exp \left(i\left(\omega_{f m}-\omega\right) t\right)-1}{\left(\omega_{f m}-\omega\right)}\right)
\end{aligned}
$$

The first term in parentheses is in resonance for our case $\left(2 \omega=\omega_{\mathrm{gf}}\right)$, so I will neglect the second term. For short times $t$, the first term $\approx i t$, so the transition probability is

$$
\left|c_{f}^{(2)}(t)\right|^{2}=\frac{(q E)^{4} x_{f m}{ }^{2} x_{m g}{ }^{2}}{\hbar^{4}\left(\omega_{m g}-\omega\right)^{2}} t^{2}
$$

It should not be surprising that the probability is proportional to $t^{2}$ because the situation is a two-photon Rabi oscillation with an effective potential of

$$
v_{1}=\frac{(q E)^{2} x_{f m} x_{m g}}{\hbar\left(\omega_{m g}-\omega\right)}
$$

The upper state probability is just 


$$
\left|c_{f}^{(2)}(t)\right|^{2}=\sin ^{2}\left(\frac{V_{1} t}{\hbar}\right)
$$

For $\mathrm{t}$ much less than an oscillation period, Equation (6) reduces to Equation (4). In this experiment, $E=4500$ esu $\left(=10^{10} \mathrm{~W} / \mathrm{cm}^{2}\right), x_{\mathrm{fm}}=3.8 \mathrm{pm}, \mathrm{x}_{\mathrm{mg}}$ $=140 \mathrm{pm}, \omega_{\mathrm{mg}}=2.5 \times 10^{15} \mathrm{sec}^{-1}, \omega=3.14 \times 10^{15} \mathrm{sec}^{-1}, \mathrm{t}=\mathrm{T}_{\mathrm{L}}=3 \mathrm{psec}$ (the laser pulse length), so $\left|c_{\mathrm{f}}^{(2)}(\mathrm{t})\right|^{2}=1.2 \times 10^{-2}$, and Equation (4) is a good approximation.

However, our experiment is concerned with more than just the transition probability to the excited state. It is the coherent superposition of that state with the ground state that can give rise to second-harmonic radiation. We therefore need to examine the evolution of the off-diagonal elements of the density matrix, $\rho_{\mathrm{fg}}(\mathrm{t})$, as well. The initial condition is $\rho_{\mathrm{fg}}(0)=0$. In the relaxation approximation,

$$
\frac{d \rho_{f g}(t)}{d t}=-i \omega_{g f} \rho_{f g}+i \frac{V(t)}{\hbar}\left(\rho_{g g}-\rho_{f f}\right)-\frac{\rho_{f g}}{T_{2}}
$$

where $T_{2}$ is the relaxation time for the off-diagonal element to decay to its equilibrium value of zero. $\left(\rho_{\mathrm{gg}}-\rho_{\mathrm{ff}}\right) \approx 1$ at all times, so if we consider a resonant $\left(2 \omega=\omega_{g f}\right)$, short $\left(T_{L} \ll T_{2}\right)$, square laser pulse applied as the perturbation $\left(V_{1}\right.$ applied for $\left.0<t<T_{L}\right)$, we get a peak magnitude of

$$
\rho_{f g}\left(T_{L}\right) \equiv \rho_{0}=\frac{i V_{1} T_{L}}{\hbar}
$$

at the end of the pulse, and is $\approx 0.11$ for the experimental values above. After the laser pulse, $V(t)=0$, but $\rho_{\mathrm{fg}}$ still evolves according to Equation (7). It therefore oscillates at $\omega_{\mathrm{gf}}$ (which is the second harmonic of the laser frequency) as it decays to zero:

$$
\rho_{\mathrm{fg}}(t)=\tilde{\rho}_{\mathrm{fg}}(t) \exp (-i \omega t)
$$

For low pressures, the relaxation approximation in Equation (7) does not hold. Instead, the inhomogeneous Doppler broadening dominates the 
decay, so $\rho_{\mathrm{fg}}$ undergoes a Gaussian decay:

$$
\tilde{\rho}_{f g}(t)=p_{0} \exp \left(\frac{-t^{2}}{T_{i}^{2}}\right)
$$

where $T_{i}$ is the inhomogeneous broadening time

$$
T_{i}=\sqrt{\frac{2 M c^{2}}{k T \omega_{g f}^{2}}} .
$$

For potassium at $T=350^{\circ} \mathrm{C}$ and the $4 \mathrm{~s}-9 \mathrm{~d}$ transition frequency, $T_{i}=370$ picoseconds.

At higher pressures, collisional dephasing starts to dominate. Assuming that the dephasing time is independent of velocity,

$$
\begin{aligned}
\tilde{\rho}_{\mathrm{fg}}(t) & =\int d \omega \exp \left(\frac{-\omega}{\omega_{d}^{2}}\right) \exp \left(\frac{-t}{T_{h}}\right) \\
& =\exp \left(-\frac{t^{2}}{T_{i^{2}}}-\frac{t}{T_{h}}\right)
\end{aligned}
$$

$T_{h}$ is the homogeneous dephasing time due to collisions:

$$
T_{h}=\frac{1}{v \sigma N}
$$

where $v$ is the average spee $d, \sigma$ is the cross section, and $N$ is the density of the colliding partner species. For sodium atoms colliding with argon, $\sigma$ has been measured ${ }^{18}$ and reaches a large maximum around $n=9$. Model decay curves of this coherent component are drawn in Figure 2 for various Argon pressures, using a $3500 \AA^{2}$ cross section like that seen in sodium.

These decays are important because they determine the time scale of a short-pulse experiment. The radiation of the coherence persists for tens or hundreds of picoseconds after the laser pulse. Thus if the symmetry of the medium changes with time, the coherence will sample the broken symmetry throughout its decay.

If the observed second-harmonic light is due to the above second- 
harmonic coherence, then Equation (12) predicts a relationship between the SHG and the Ar pressure. The detectors are slow compared to the coherence time of the atoms, so they only measure the signal integrated over the entire decay. If $\rho_{f g}$ could radiate directly, then Equation (12) predicts that the observed signal would be proportional to $\frac{1}{N_{A r}}$ for high Ar pressures (where $T^{\text {h }}$ dominates) and independent of $N_{A r}$ for low Ar pressures (where $T_{i}$ dominates). A calculated curve is shown in Figure 3 . If $\rho_{\mathrm{fg}}$ cannot radiate uniformly in time (as would happen for a slowly changing symmetry), then the shape of this curve would be changed.

\section{Radiation of a Second-Harmonic Coherence}

The macroscopic polarization of the medium is determined from the density matrix in the usual way: ${ }^{5}$

$$
\begin{gathered}
\langle\overrightarrow{\mathrm{P}}>=\operatorname{Tr}(\rho \overrightarrow{\mathrm{P}}) \\
=-\operatorname{Ne} \sum_{\mathrm{m}}<\mathrm{m}|\rho \overrightarrow{\mathrm{x}}| \mathrm{m}>
\end{gathered}
$$

Using a $3 \times 3$ density matrix with the $g, f$, and $r$ states represented, the real $\vec{x}$ matrix can be represented as

$$
\vec{x}=\left(\begin{array}{ccc}
0 & 0 & x_{\mathrm{gr}} \\
0 & 0 & x_{\mathrm{fr}} \\
x_{\mathrm{gr}} & x_{\mathrm{fr}} & 0
\end{array}\right)
$$

Then the polarization is

$$
-\mathrm{Ne} \operatorname{Tr}(\rho \vec{x})=-\operatorname{Ne}\left\{2 \operatorname{Re}\left(\rho_{\mathrm{rg}}\right) x_{\mathrm{gr}}+2 \operatorname{Re}\left(\rho_{\mathrm{rf}}\right) x_{\mathrm{fr}}\right\} .
$$

As expected, $\rho_{\mathrm{gf}}$ does not contribute directly to the dipole moment of the medium. It can contribute indirectly if it is mixed with $\rho_{\mathrm{rg}}$ or $\rho_{\mathrm{rf}} \cdot \rho_{\mathrm{rg}}$ and $\rho_{\text {rf }}$ can become nonzero through an interaction that breaks the symmetry of the Hamiltonian:

$$
\frac{d \rho_{\mathrm{rg}}(\mathrm{t})}{\mathrm{dt}}=\frac{-\mathrm{i}}{\hbar}\left[\mathrm{H}_{0}+\mathrm{H}_{\mathrm{int}} \rho\right]_{\mathrm{rg}},
$$


where $\mathrm{H}_{0}$ is the spherically symmetric atomic Hamiltonian and $\mathrm{H}_{\text {int }}$ is the symmetry-breaking perturbation. Since $p$ describes the ensemble average of atomic systems, $\mathrm{H}_{\mathrm{int}}$ is the ensemble average of Hamiltonians that act on those systems.

Once a polarization is established in the medium, the light generated is calculated from Maxwell's wave equation. In the case of a beam traveling in the $z$-direction, the wave equation takes the form 5

$$
\frac{\partial}{\partial z} E(\omega, z)=\frac{2 \pi i \omega}{c} P(\omega, z) \exp (i \Delta k z) \text {. }
$$

The phase mismatch, $\Delta \mathrm{k}$, is dominated by the potassium polarizability, and for $\mathrm{N}=10^{16}$ atoms $/ \mathrm{cm}^{3}, \Delta \mathrm{k}=0.7 \mathrm{~cm}^{-1}$. Integrating Equation (18) for a polarization constant in $\mathrm{z}$ gives maxima of the electric field of

$$
\mathrm{E}\left(\omega, \mathrm{z}_{\max }\right)=\frac{2 \pi \omega}{\Delta \mathrm{kc}} \mathrm{P}(\omega) \text {. }
$$

A polarization of $10^{-9}$ esu radiating for $100 \mathrm{psec}$ is required in order to account for our observed signal.

Next, I will consider effects that could generate second-harmonic light, either directly or by inducing the above coherence to radiate. Most models will be eliminated because they cannot produce the observed amount of polarization.

\section{A. Fluorescence and superradiance}

Because several other wavelengths are observed with a 4s-9d tuning, the mechanism of simple fluorescence must be considered. Individually, the atoms would radiate as quadrupoles, and their radiation would add incoherently in all directions. Decays via nearby states would then account for the other frequencies we observe.

The largest theoretical argument against such an effect is that the states 
are oidy quadrupole-coupled (in the case of $9 \mathrm{~d}$ ) to the ground state. Therefore their primary decay path will be to dipole-coupled intermediate $\mathrm{p}$-states. However, we have not looked for $9 \mathrm{~d}-\mathrm{np}$ radiation, so experimentally we cannot make a direct comparison. Other characteristics of incoherent decay would be its uniform radiation into all directions, and persistence after the laser pulse for the excited state lifetime of $9 \mathrm{~d}$, which is on the order of microseconds long. Our observation of a collimated, prompt response refutes this mechanism as an explanation.

The related phenomenon of superfluorescence ${ }^{5}$ occurs when an inverted population of atoms couple together strongly via the radiation field. They can then radiate coherently, and if the spatial distribution of excited atoms is a long cylinder (as in this experiment), the radiation would be collimated along the cylinder. A single lobe would be emitted in each direction out of the cylinder. The radiation can also occur in times much shorter than the normal lifetime of the excited state.

The weak coupling between radiation and the 4s-9d transition makes this mechanism a hardly credible one. Not only are the couplings stronger between $9 \mathrm{~d}$ and $\mathrm{np}$, but the populations between these states will be inverted. The estimated $1 \%$ excitation to the $9 \mathrm{~d}$ state is not inverted, and therefore cannot become superradiant. Thus if any superfluorescence were to occur in this system, it would occur at a different frequency from our observed secondharmonic. Additional experimental inconsistency with this model comes from the two-lobed structure evident in the SHG.

\section{B. Multipole contributions}

Because the laser is intense and the response is weak, it is natural to consider whether higher-order multipoles could account for some of the 
observed signal. The 4s-11s transition has no multipole moment in any order, so this mechanism would not directly account for the $11 \mathrm{~s}$ response. The following calculation examines the multipole radiation from the $9 \mathrm{~d}$ state.

First, the magnetic dipole is easily eliminated because the magnetic dipole moment will be zero in a linearly polarized beam:

$$
\vec{M}(2 \omega)=\chi_{m}\left(\vec{E}_{1}(\omega) \times \vec{E}_{2}(\omega)\right)=0 \text { if } \vec{E}_{1}(\omega)=\vec{E}_{2}(\omega)
$$

The electric quadrupole contribution is much harder to eliminate. There are two types of quadrupolar contributions to SHG. One type concerns a quadrupolar interaction with the incident radiation, and the other concerns quadrupolar generation of the outgoing radiation. The first type generates a polarization in a mediur: proportional to a quadrupole moment of the input field:

$$
\overrightarrow{\mathbf{P}}(2 \omega)=\stackrel{\leftrightarrow}{\chi} \underset{q}{p}: \vec{E}_{1}(\omega) \vec{\nabla} \vec{E}_{2}(\omega)
$$

while the second type generates a quadrupole moment in the medium:

$$
\stackrel{\leftrightarrow}{\mathbf{Q}}=\stackrel{\leftrightarrow}{\chi}_{q}^{q}: \overrightarrow{\mathbf{E}}_{1}(\omega) \overrightarrow{\mathbf{E}}_{2}(\omega)
$$

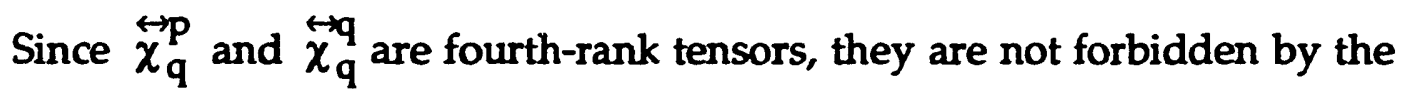
centrosymmetry of the vapor. Explicit equations for these tensors are given by Bethune in terms of sums over the atomic resonances. At the $4 s-9 d$ transition, only $\stackrel{\leftrightarrow}{\chi}_{q}^{q}$ shows a resonant enhancement, as would expected from the $s-d$ selection rules.

This contribution can also be thought of as the direct radiation of the off-diagonal element generated in Equation (8). While a d-state is not coupled with the ground state via dipole radiation, it is coupled via quadrupole radiation. In effect, the quadrupole moment $\stackrel{\leftrightarrow}{\mathbf{Q}}$ given in Equation (22) has summed over the individual quadrupoles. The effective polarization of a 
quadrupole moment is due to its gradient:

$$
\overrightarrow{\mathbf{P}}(2 \omega)=\vec{\nabla} \cdot \stackrel{\leftrightarrow}{\mathbf{Q}}=\vec{\nabla} \cdot \stackrel{\leftrightarrow}{\chi}_{q}: \overrightarrow{\mathbf{E}}_{1}(\omega) \overrightarrow{\mathbf{E}}_{2}(\omega)
$$

Centrosymmetry places several restrictions on the elements of $\stackrel{\leftrightarrow}{\chi}_{q^{q}}{ }^{21}$ From simple reflections and $90^{\circ}$ rotations, it can be seen that there are only four distinct elements:

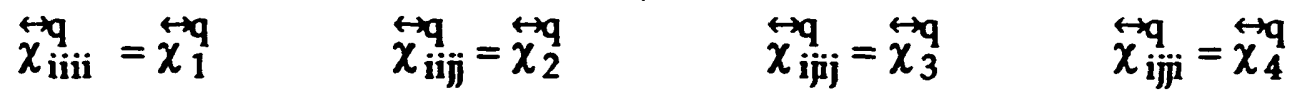

Symmetry for small rotations also gives the relation

$$
\stackrel{\leftrightarrow q}{x_{1}}=\stackrel{\leftrightarrow q}{x_{2}}+\stackrel{\leftrightarrow q}{x_{3}}+\stackrel{\leftrightarrow}{x_{4}} \text {. }
$$

For second-harmonic generation, $\overleftrightarrow{\chi}_{q}^{q}$ must be symmetric with respect to an exchange of input fields, giving

$$
\overleftrightarrow{\leftrightarrow}_{3}^{q}=\stackrel{\leftrightarrow}{\chi}_{4}^{q}
$$

The quadrupolar radiation from a Gaussian cylinder of excited atoms might well be a collimated, two-lobed beam, similar to the SHG observations. But as Bethune worked out, ${ }^{22}$ the gradients of quadrupole moment along the sides of the cylinder can radiate only weakly. This results from the full vectorial expression of a linearly polarized Gaussian beam traveling along the $z$ axis:

$$
\vec{E}=\vec{E}_{0} \psi\left(\hat{e}_{x}-\frac{i \theta \times \zeta}{R} \hat{e}_{z}\right)
$$

where $R$ is the minimum beam waist, $\hat{e}_{\mathbf{x}}$ and $\hat{e}_{\mathbf{z}}$ are unit vectors in the $\mathbf{x}$-and z- directions, respectively, and

$$
\begin{gathered}
\psi(r, z)=\zeta \exp \left(i k z-\zeta \frac{r^{2}}{R^{2}}-i \omega t\right) \\
\zeta(z)=\left(1-\frac{i z}{z_{0}}\right)^{-1} \\
z_{0}=\frac{k^{2}}{2} \quad \text { (the confocal parameter) } \\
\theta=\operatorname{Tan}^{-1}\left(\frac{2}{k R}\right) \quad \text { (the beam divergence half-angle). }
\end{gathered}
$$


The usually-neglected z-component of the electric polarization is necessary to satisfy Gauss' Law for a divergenceless electric field in free space, which the above approximation satisfies to order $\theta^{3}$. The $z$-component is quite small for a loosely focused beam, but it plays an important role in quadrupole radiation from a centrosymmetric medium. The effective polarization in the i-direction is then worked out from

$$
P_{i}=\stackrel{\leftrightarrow}{\chi_{i j k l}} \frac{\partial}{\partial x_{j}}\left(E_{k} E_{l}\right) \text {. }
$$

The algebra shows that both $P_{x}$ and $P_{z}$ are non-zero, with contributions from all of the distinct elements of $\stackrel{\leftrightarrow}{\chi}$; however, the polarization can only radiate if it has a curl. The curl of our calculated $\vec{P}$ is zero up to order $\theta^{2}$ under the sy umetry relation defined in Equation (25). The remaining contribution is ${ }^{17}$

$$
P(2 \omega)=\chi_{b}^{(2)}(2 \omega) \frac{\theta^{2} \zeta}{4} \frac{\partial}{\partial x}\left(E^{2}\right)
$$

where

$$
\begin{aligned}
\chi_{b}^{(2)}(2 \omega)=\frac{N e^{3}}{2 \hbar^{2}} \sum_{n} & \frac{<s\left|z^{2}\right| 9 d><9 d|z| n p><n p|z| 4 s>}{\left(\omega_{4 s, 9 d}-2 \omega_{\mathcal{L}}\right)\left(\omega_{4 s, n p}-\omega_{\mathcal{L}}\right)} \\
& \approx 6 \times 10^{-18} \approx s u .
\end{aligned}
$$

For this experiment, $\theta=10^{-4}$, so this polarization is only $3 \times 10^{-18} \mathrm{esu}$, nine orders of magnitude smaller than the observed SHG.

The amplified laser beam is not Gaussian, and is closer in form to a flat-topped beam due to saturation in the center of the amplifiers. However, the contribution of higher order transverse modes also cancels out up to order $\theta^{2}$, and the remainder is similar in magnitude to the Gaussian contribution above. Thus quadrupole contributions cannot account for the observed signal. 


\section{Collisional mechanism}

A number of authors $10,11,15,23,24$ have proposed that atomic collisions play the part of the symmetry-breaking $\mathrm{H}_{\text {int }}$ in Equation (17). The experimental evidence from this picosecond study refutes this proposition. There are two types of collisions: those between distinct partners (K and $\mathrm{Ar}$ ), and those between identical partners ( $\mathrm{K}$ and $\mathrm{K}$ ). Each type will be treated below.

Most previous experiments were done in an oven with a central heating zone. The metsi vapor was kept at partial pressures $\leq 1$ Torr with a noble buffer gas of 10-1000 Torr throughout the oven. Thus the primary collision partner was a noble gas atom. It is clear that a mixed-pair collision will break the microscopic symmetry of an individual alkali atom; however, such collisions will not break the macroscopic symmetry of the vapor because the individual collisions will take place at random orientations. Thus coherent SHG cannot be explained by simple collisions. Couched in terms of Equation (17), the $\mathrm{H}_{\text {int }}$ acting on the entire ensemble of excited atoms is the average of all the randomly oriented collisions. It has no direction associated with it, so it is not a vector, and cannot mix terms of opposite parity. Therefore collisions cannot induce $\rho_{\mathrm{fg}}$ to radiate.

By considering the effect of the nonuniform laser excitation, the macroscopic symmetry can be broken. Because more atoms are excited at the center of the beam that at the edge, there will be a gradient in the excited state population density from the edge to the center of the beam. But even this gradient cannot change the scalar quality of $\mathrm{H}_{\text {int }}$ because the noble gas collision partners are still randomly oriented about any small ensemble of excited atoms. 
It is possible that an unknown collision mechanism induces the radiation, but predictions can still be made about its behavior. Any $\mathrm{H}_{\text {int }}$ would depend on $\mathbf{N}_{\mathrm{Ar}}$, so the $\mathbf{N}_{\mathrm{Ar}}$ dependence predicted in Figure 3 would be altered. An extremely conservative estimate of the upper limit for a significant impact is the experimentally measured dephasing impact parameter, 50 Angstroms. At 10 Torr, the noble gas atoms are more than 200 Angstroms apart on average so only $10 \%$ of the excited atoms will be undergoing a collision at a time. Increasing the noble gas density should therefore increase the number of significant collisions and consequently the SHG radiation. This increase would slow the decrease in signal expected from the faster dephasing of the signal at higher pressures.

In our experiments, however, we observe a faster decrease in signal with Ar pressure than expected from sim yle dephasing (see Figure 16 in Chapter 4). In addition, we lowered the Ar pressure to less than 1 Torr, bringing the oven into a heat pipe mode ${ }^{25}$ where the central potassium vapor pressure equaled the pressure applied by the Ar. In this mode, Ar is excluded from the center of the oven entirely, since the total pressure throughout the oven must remain constant. We saw no decrease in signal when we entered this mode, so clearly the Ar can play no direct part in the SHG radiation.

Collisions between potassium atoms could also generate coherent SHG. In this case, an individual collision is still centrosymmetric, and the pair can be considered a quadrupole, because the two atoms are identical. However, the excitation gradient introduces a gradient of the quadrupole density, which acts as an effective dipole.

The collisions mix the excited 9d state with other states of the atom:

$$
19 d^{\prime}>=19 d>+\Sigma \phi_{n}(r)|n\rangle
$$


where $\phi_{n}(r)$ is the mixing parameter, and depends strongly on the distance $r$ between the colliding atoms. As an induced dipole-induced dipole effect, it wiil be related to the van der Waals interaction and its $1 / 1^{6}$ dependence. The second-harmonic polarizability of each atom is then

$$
\stackrel{\leftrightarrow}{\beta}(2)(r)=\sum_{m} e^{3} \phi_{n}(r) \frac{\langle 4 s|\vec{x}| n\rangle\langle 9 d|\vec{x}| n p>\langle n p|\vec{x}| 4 s\rangle}{\hbar^{2}\left(\omega_{4 s, 9 d}-2 \omega_{\nu}\right)\left(\omega_{4 s, n p}-\omega_{I}\right)}
$$

and the magnitude of the quadrupole moment of the pair is

$$
Q(\mathbf{r})=\mathbf{r} \overleftrightarrow{\boldsymbol{\beta}}(2)(\mathbf{r}): \vec{E} \vec{E}
$$

The effective polarization is the gradient of this moment across the beam,

$$
\overrightarrow{\mathbf{P}}(2 \omega)=\frac{\langle\mathrm{r} \stackrel{\leftrightarrow}{\beta}(r)>}{6 \mathbf{R}} \overrightarrow{\mathbf{E}} \overrightarrow{\mathbf{E}}
$$

where 6 is a geometric factor from averaging over quadrupole orientations, $R$ is the radius of the laser beam, and the angle brackets denote averaging over all collisions taking place.

A crude estimate of the average assumes $\phi(r)$ to be constant out to an effective collision radius $r_{\max }$, and zero beyond $r_{\max }$. The number of atoms undergoing such collisions will be $\left(r_{\max } / r_{a v e}\right)^{3}$. Even by assuming the maximum possible $\beta$ of 0.5 , and allowing for $r_{\max }=50 \AA$, the effective polarization is three orders of magnitude smaller than the experiment observes. A more realistic estimate for $\mathrm{I}_{\max }$ might be five times smaller, and because the polarization deperids on $\mathrm{r}_{\max }^{4}$, the actual polarization will be much smaller than this crude estimate. Thus collisions between potassium atoms cannot account for the magnitude of our observed signal by several orders of magnitude.

\section{Free electron nonlinearity}

The nonuniform excitation in the vapor will also create a nonuniform 
distribution of free electrons due to photoionization. As Shen calculated in his introduction, ${ }^{5}$ a nonuniform plasma will act as a nonlinear medium and generate a second-harmonic polarization

$$
\overrightarrow{\mathbf{P}}(2 \omega)=\frac{\mathrm{e}^{3}}{4 \mathrm{~m}^{2} \omega^{4}}\left(\vec{\nabla} \mathrm{N}_{e} \cdot \overrightarrow{\mathrm{E}}\right) \overrightarrow{\mathrm{E}}
$$

This polarization will only exist while the laser pulse is present because the free electron nonlinearity does not have the sharp resonance of the atomic response. With the values of $\mathrm{N}_{\mathrm{e}}=10^{12} \mathrm{~cm}^{-3}, \mathrm{R}=100 \mu \mathrm{m}$, and $\mathrm{E}=4500 \mathrm{esu}$, the calculated amount of polarization is five orders of magnitude too small to account for the observed signal, so we eliminate this model as well. Others' experiments found similar discrepancies.

\section{E. Amplification due to $\chi^{(5)}$}

Another interesting possible mechanism is due to a dipole-allowed $\chi^{(5)}(2 \omega=\omega+\omega+\omega+\omega-2 \omega)$, whose transitions are diagrammed in Figure 4. It would be a high-order parametric-conversion mechanism since light at $2 \omega$ is required to generate the polarization. In this experiment, it would be singly resonant with the narrow atomic $9 \mathrm{~d}$ resonance and doubly resonant with continuum states. However, it is not resonant with the $9 \mathrm{~d}$ state in the two$2 \omega$-photon transition from the continuum to the ground state. While the $9 \mathrm{~d}$ state is at the right energy, it does not obey dipole selection rules, so it cannot contribute.

It is fairly difficult to estimate the strength of $\chi^{(5)}$ theoretically. However, this model makes some predictions that are easy to test experimentally. The clearest difference is in the intensity dependence of the signal. As a parametric conversion, the signal undergoes exponential gain within the active medium. The gain is proportional to $I^{4}$, so the intensity 
dependence should be exp( $\left(I^{4}\right)$. This extremely strong dependence would appear as an upward curvature on a semi-log graph and as a stronger upward curvature on a log-log graph. The observed signal has a downward curvature on a log-log graph for all measurable intensities.

The observed output structure also contradicts this mechanism. The strong intensity deper.dence would indicate a beam strongly peaked in the center. Instead, the output has two lobes and a low intensity in the center. If the low center intensity were due to phase mismatch, then the output power would increase dramatically as the potassium density is lowered. It does not.

Another characteristic of $\chi^{(5)}$ effects would be its output spectrum. It would have an additional downward resonance with any p-state, so its effect would be resonantly enhanced at specific frequencies different from $2 \omega$. We already observe very strong signals of $\approx 10,000$ counts/shot at the $10 \mathrm{p}-4 \mathrm{~s}$ transition (which we attribute to dipole-allowed four-wave mixing, diagrammed in Figure 1), so the resonant $\chi^{(5)}$ should generate strong signal at $4 \omega-\omega_{10}$, which corresponds to a wavelength of 2960 Angstroms. We do see some signal at this wavelength, but it is only 10 counts/shot in spite of the stronger generating field and the resonantly enhanced $\chi^{(5)}$. The increased phase mismatch at s-p transition frequencies makes a quantitative comparison difficult, but this weak signal makes the non-resonant $\chi^{(5)}$ an unlikely candidate for SHG.

\section{F. Electric Field due to Ionization}

If a dc electric field is present, then we have $H_{i n t}=e \vec{E}_{d c} \cdot \vec{x}$ and Equation (17) becomes

$$
\frac{d \rho_{\mathrm{rg}}(t)}{\mathrm{dt}}=\mathrm{i} \omega_{\mathrm{gr}} \rho_{\mathrm{rg}}+\frac{i}{\hbar} \text { e } E_{\mathrm{dc}} x_{\mathrm{gr}}\left(\rho_{\mathrm{rr}}-\rho_{\mathrm{gg}}\right)-\frac{i}{\hbar} \text { e } E_{\mathrm{dc}} x_{\mathrm{fr}} \rho_{\mathrm{gf}} .
$$


$\rho_{\mathrm{gf}}$ will act as the driving force for this oscillator, $\rho_{\mathrm{rg}}$. Because $\rho_{\mathrm{gf}}$ oscillates at $\omega_{\mathrm{gf}} \rho_{\mathrm{rg}}$ will also have an oscillatory component at $\omega_{\mathrm{gf}}$, and Equation (36) can be solved with:

$$
\rho_{\mathrm{rg}}(t)=A \exp \left(i \omega_{\mathrm{gf}} t\right)+B
$$

where

$$
\begin{gathered}
A=\frac{e E_{d c} x_{f r} \tilde{\rho}_{f g}(t)}{\hbar\left(\omega_{g f}-\omega_{g r}\right)} \\
B=\frac{e E_{d c} x_{g r}\left(\rho_{g g}-\rho_{\pi r}\right)}{\hbar \omega_{g f}}
\end{gathered}
$$

Because the driving frequency, $\omega_{\mathrm{gf}}$, is close to the natural oscillator frequency, $\omega_{g r}$, the factor A can be relatively large.

The polarization of the medium at the laser's second harmonic, $2 \omega=\omega_{\mathrm{gf}}$, due to $\rho_{\mathrm{fg}}$, is then

$$
P(2 \omega)=2 N_{K} e A x_{g r}=\frac{2 e^{2} E_{d c} x_{f r} x_{g r} \tilde{\rho}_{f g}(t) N_{K}}{\hbar\left(\omega_{g f}-\omega_{g r}\right)}
$$

The polarization due to $p_{\mathrm{rf}}$ is identical except that it has the much larger factor $\omega_{\mathrm{rg}}$ in the denominator of $\mathrm{A}, \mathrm{so}$ it is neglected. For a moderate dc electric field of 0.1 esu (see the estimates made in Chapter 4) and potassium density $10^{16}$ atoms $/ \mathrm{cm}^{3}$, the second-harmonic polarization is $7.8 \times 10^{-9} \mathrm{esu}$.

The result is not exact because it assumes a steady state, whereas in reality $E_{d c}$ and $\rho_{g f}$ both change slowly with time, either growing or decaying in a time on the order of tens of picoseconds. However, the only effect of such transients would be some kind of ringing or heating that would not significantly affect the total integrated radiation. The estimated dc-fieldinduced polarization is ten times larger than needed to account for our signal, but details of the transverse beam structure may account for the discrepancy.

Other characteristics of field-induced radiation also fit the observations. 
It can induce radiation of both s-d and s-s transitions. The emitted light would be both prompt and collimated. The field is zero on the axis of the laser, so SHG would be zero there as well. Further details require the more exact description of the electric field given in the next chapter.

\section{Conclusion}

In this chapter I have described a number of mechanisms that could generate SHG in an atomic vapor. The restrictions placed on these mechanisms by the symmetry of the medium have been severe. In most cases, the effectiveness of the mechanism is far below that which we observe in experiment. Other characteristics of the mechanisms also differ from those observed. On the other hand, the mechanism of a moderate dc electric field fits many of the characteristics of the observations. In the next chapter, I will examine the details of how this field could arise and make detailed comparisons with our experimental observations. 


\section{References}

1. S. Dinev, J Phys B: At. Mol. Opt. Phys. 21 : 1681 (1988).

2. K. Hakuta, L. Marmet and B. P. Stoicheff, Phys. Rev. Lett. 66 (5): 596 (1991).

3. T. Mossberg, A. Flusberg and S. R. Hartmann, Opt. Commun. 25 (1): 121 (1978).

4. J.-Y. Zhang, H.-T. Zhou and P. Jin, in conference proceedings of Resonance Ionization Spectroscopy, T. B. Lucatorto, J. E. Parks, Eds. (Institute of Physics, Gaithersburg, Maryland, USA, 1988) \#4, p. 29.

5. Y. R. Shen, The Principles of Nonlinear Optics (John Wiley \& Sons, New York, 1984), pp. 8, 16, 28, 71, 407.

6. A. Flusberg, T. Mossberg and S. R. Hartmann, Phys. Rev. Lett. 38 (2): 59 (1977).

7. D. S. Bethune, R. W. Smith and Y. R. Shen, Phys. Rev. A 17 (1): 277 (1978).

8. J. Okada, Y. Fukuda and M. Matsuoka, J. Phys. Soc. Jap. 50 (4): 1301 (1981).

9. J. Manners and A. Durrant, Opt. Commun. 58 (6): 389 (1986).

10. M.-H. Lu and L.-H. Tsai, J. Phys. B: At. Mol. Opt. Phys. 23 (5): 921 (1990).

11. S. Dinev, A. Guzman de Garcia, P. Meystre, R. Salomaa and H. Walther, in conference proceedings of Laser Spectroscopy, H. P. Weber, W. Luthy, Eds. (Berlin, Germany: Springer, 1983) \#VI, p. 183.

12. J. Bokor, R. R. Freeman, R. L. Panock and J. C. White, Opt. Lett. 6 (4): 182 (1981).

13. W. Jamroz, P. E. LaRocque and B. P. Stoicheff, Opt. Lett. 7 (4): 148 (1982).

14. K. Miyazaki, H. Sakai and S. Takuzo, IEEE J Quant Electron. QE-22 (12): 2266 (1986).

15. J.-Y. Zhang and K. T. Lu, J. Phys. B: At. Mol. Phys. 20 : 5065 (1987). 
16. J.-H. Tsai and M.-H. Lu, Appl. Phys. B 51 : 386 (1990).

17. D. S. Bethune, Phys. Rev. A 23 (6): 3139 (1981).

18. T. F. Gallagher, S. A. Edelstein and R. M. Hill, Phys. Rev. A 15 (5): 1945 (1977).

19. M. S. Malcuit, R. W. Boyd, W. V. Davis and K. Rzazewski, Phys. Rev. A 41 (7): 3822 (1990).

20. S. Augst, D. D. Meyerhofer, C. I. Moore and J. Peatross, in conference proceedings of Fsec to Nsec High-intensity lasers and applications, (SPIE, 1990) \#1229, ץ. 152.

21. P. Guyot-Sionnest and Y. R. Shen, Phys. Rev. B 38 (12): 7985 (1988).

22. D. S. Bethune, Opt. Lett. 6 (6): 287 (1981).

23. A. Elçi and D. Depatie, Phys. Rev. Lett. 60 (8): 688 (1988).

24. S. P. Tewari and S. P. Tewari, in conference proceedings of Coherence and Quantum Optics VI, J. H. Eberly, Eds. (Plenum Press, New York, 1989) \#VI, p. 1153.

25. C. R. Vidal and J. Cooper, J. Appl. Phys. 40 (8): 3370 (1969). 


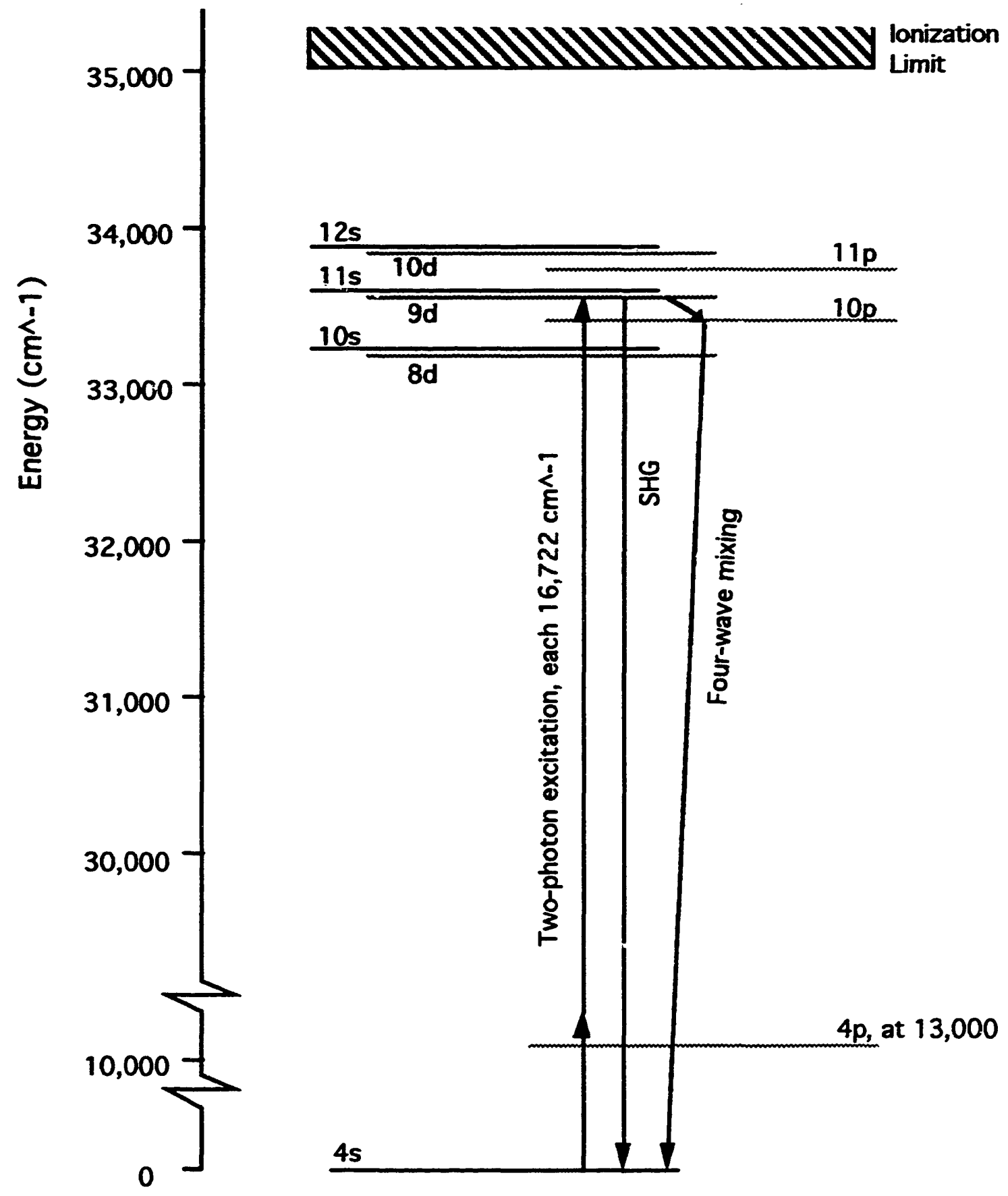

Figure 1 - Level diagram for potassium showing the relevant atomic energy levels. Typically, the laser is tuned to the two-photon $4 \mathrm{~s}-9 \mathrm{~d}$ resonance (two upward arrows). SHG appears as the single downward arrow.

Four-wave mixing, a strong allowed process with output near the SHG is shown as the two angled downward arrows. 


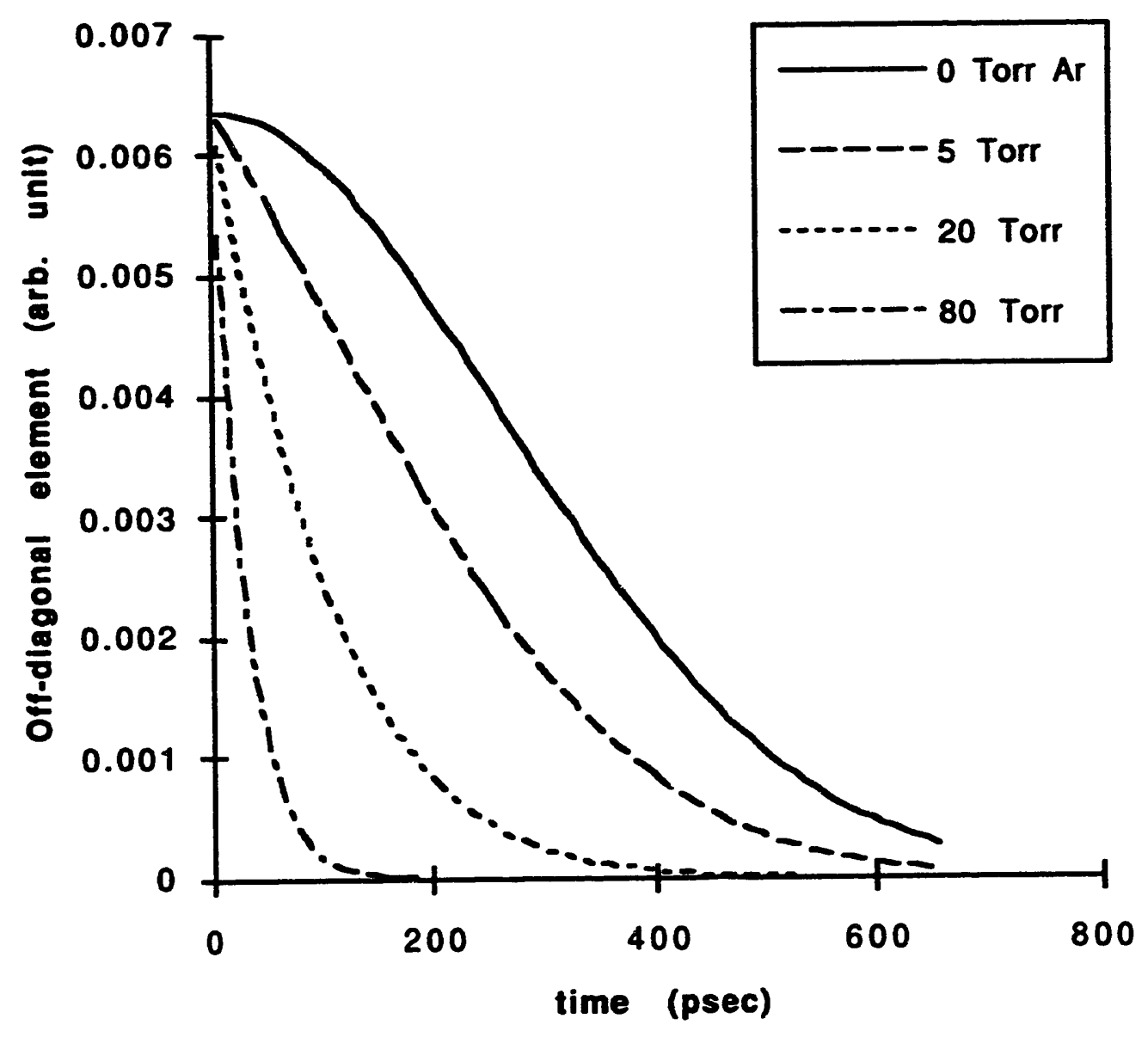

Figure 2 - Decay with time of the 4s-9d coherence for various Ar pressures. 


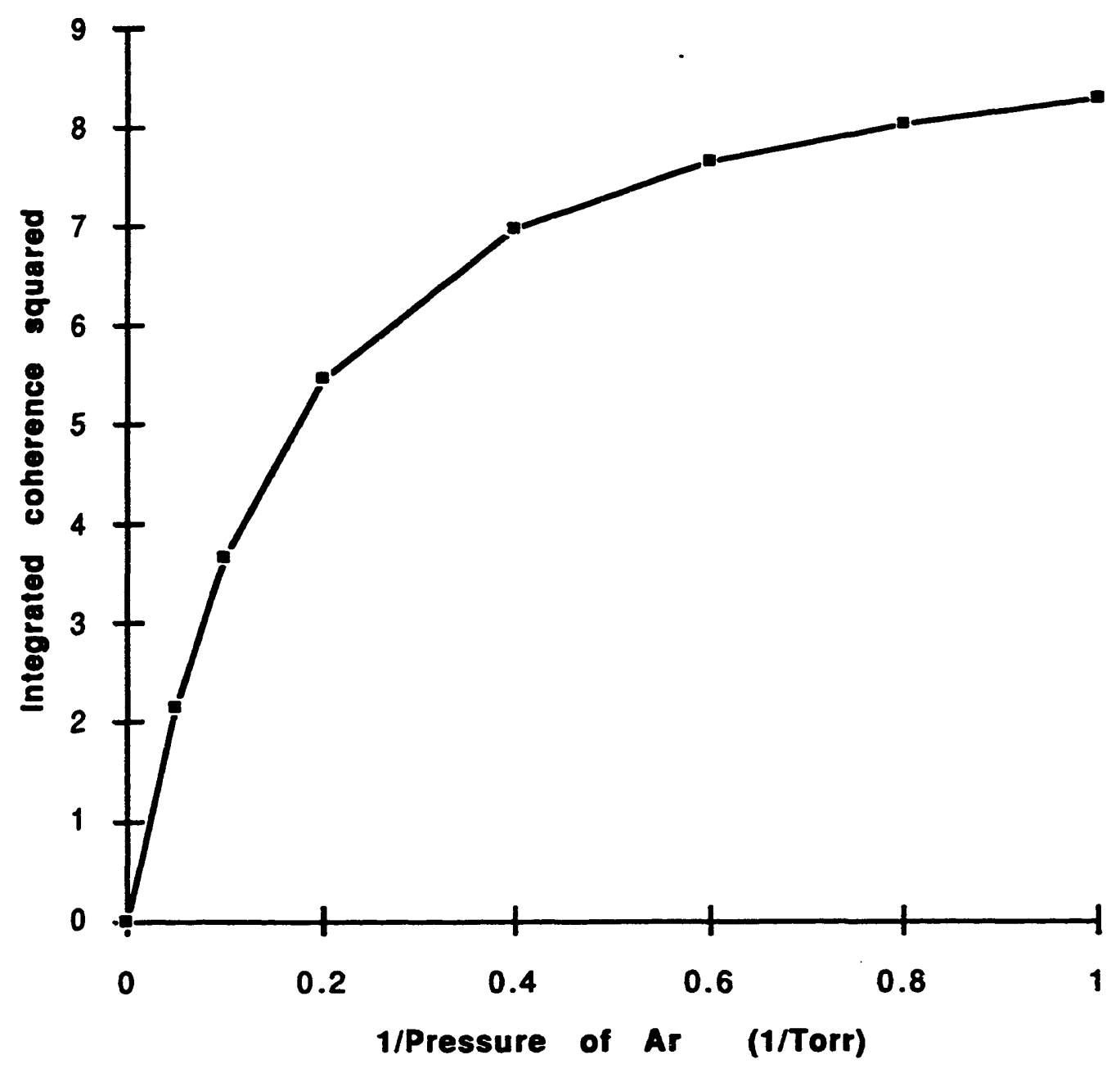

Figure 3 - Integrate signal dependence on Ar pressure, assuming that the coherence in Figure 2 can radiate with constant efficiency. 


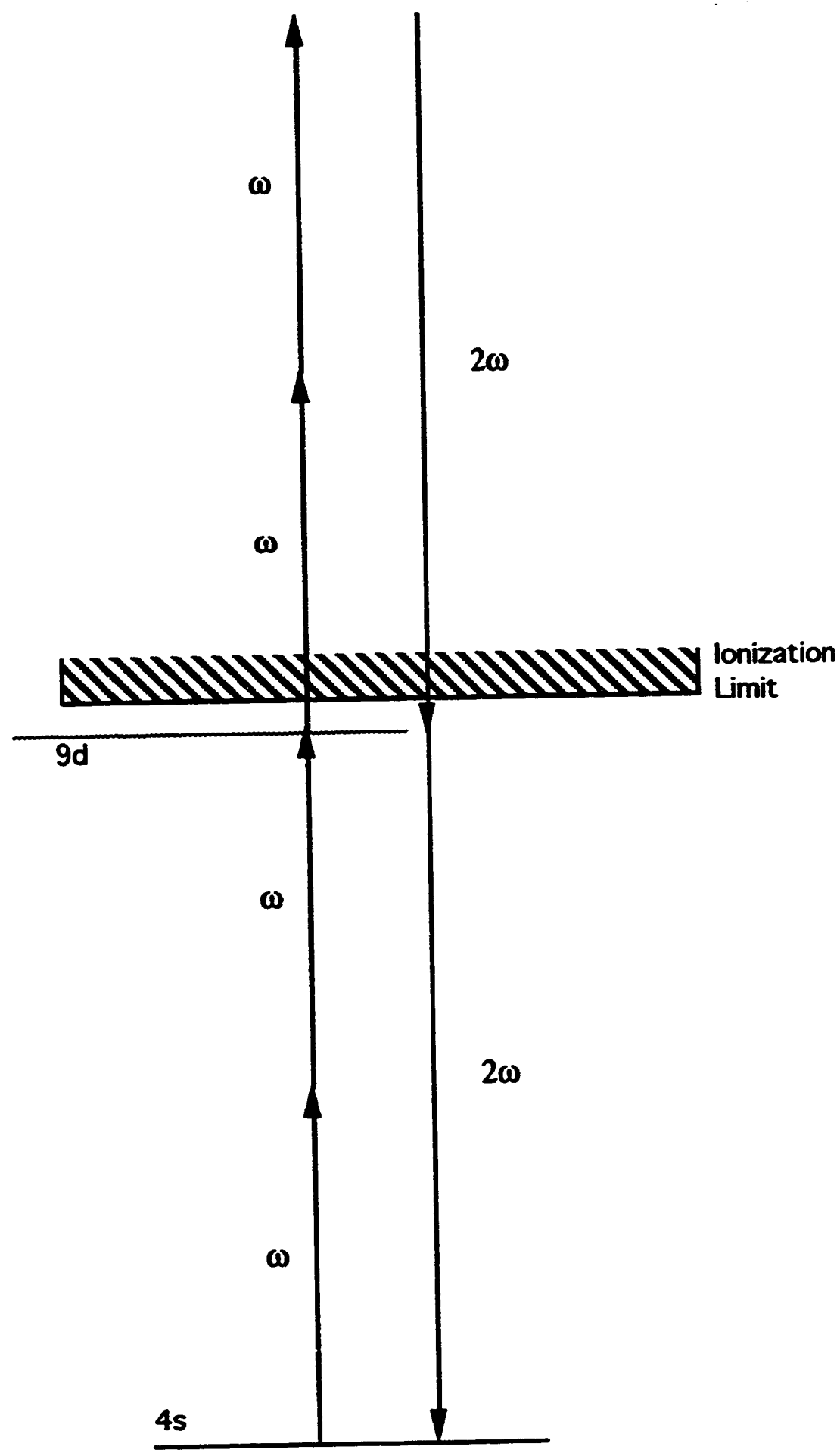

Figure 4 - Level diagram for the proposed $\chi^{(5)}$ process. Only the upward 4s-9d transition is resonant, since the downward one-photon transition at that same energy is not dipole allowed. 


\section{Results from the SHG in Vapor Experiment}

\section{Introduction}

In the remaining model of dc-field-induced SHG, I have not yet specified how the dc electric field develops from the ionization in the laser beam. Even in a simplest picture of ionization, the development may have a number of different characteristics depending on the number, initial distribution, and environment of the ionized electrons. I discuss these characteristics below and compare them with our observed results in detail.

\section{Evolution of a model dc Electric Field}

In this sectior., a fairly simple model is used to determine the prime characteristics of the dc electric field expected from ionization along a Gaussian beam. Some of the material has been drawn from an excellent paper by Bethune, ${ }^{1}$ but much of it was deduced for this experiment when the data demanded more details. For low amounts of ionization, a Gaussian laser beam will produce a Gaussian cloud of ionized electrons

$$
N_{e}(r, 0)=N_{0} \exp \left(\frac{-r^{2}}{R_{i}^{2}}\right)
$$

where $\mathbf{R}_{\mathbf{i}}$ is the radius of the ionization cloud and $\mathbf{N}_{\mathbf{0}}$ is the central peak density of electrons. An equal number and distribution of ions $\mathrm{N}_{\mathbf{i}}(\mathrm{r}, 0)$ will also be produced. Since potassium ions at $350^{\circ} \mathrm{C}$ travel only $1 \mu \mathrm{m} / \mathrm{nsec}$, their movement is negligible for the beam radii $(>50 \mu \mathrm{m})$ and delay times $(<1 \mathrm{nsec})$ used in this experiment. They are therefore be assumed to remain fixed. If three photons are required for ionization, then $R_{i}=\frac{R}{\sqrt{3}}$ where $R$ is the radius 
of the laser beam. The velocities of the released electrons are assumed to be spherically symmetric. In the case of three-photon photoionization, their initial kinetic energy is $2.1 \mathrm{eV}$, and their speed is $1.0 \mu \mathrm{m} / \mathrm{psec}$.

An exact treatment uses the Boltzmann transport equation governing the distribution of electrons, $\vec{f}(\vec{r}, \vec{v}, t)$, in the phase space, $\vec{r}, \vec{v})$,

$$
\frac{\partial f}{\partial t}+\vec{\alpha}(\vec{r}, \vec{v}) \cdot \vec{\nabla}_{v} f+\vec{v} \cdot \vec{\nabla}_{r} f=-\frac{f-f_{0}}{\tau_{c}}
$$

where $\vec{\alpha}(\vec{r}, \vec{v})$ is an acceleration vector, $f_{0}$ is the equilibrium distribution in phase space, and $\tau_{c}$ is the phenomenological collision time that brings about that equilibrium.

The spatial distribution of electrons is calculated at any time by

$$
N_{e}(\vec{r}, t)=\int d^{3} \vec{v} f(\vec{r}, \vec{v}, t)
$$

This then leads to an electric field via Gauss' Law:

$$
E(r, t)=\frac{4 \pi e}{r} \int_{0}^{r} d r^{\prime} r^{\prime}\left(N_{i}\left(r^{\prime}, 0\right)-N_{e}\left(r^{\prime}, t\right)\right) .
$$

where the cylindrical symmetry of the problem has been utilized. Since the initial electron and ion spatial distributions are equal, they cancel in the above equation at $t=0$. The remaining problem is to calculate the change in electron distribution $\Delta N_{e}(r, t)=N_{e}(r, t)-N_{e}(r, 0)$, and

$$
E(r, t)=\frac{-4 \pi e}{r} \int_{0}^{r} d r^{\prime} r^{\prime} \Delta N_{e}\left(r^{\prime}, t\right) \text {. }
$$

\section{A. Ponderamotive potential}

The laser beam exerts a force on the electrons due to the ponderamotive potential. This force was used to account for the SHG observed in other psec experiments. The depth of this potential is

$$
U_{p}=\frac{e^{2} E^{2}}{2 m \omega^{2}}
$$


which is $\frac{k T}{2}$ for a tightly focused beam of $10^{12} \mathrm{~W} / \mathrm{cm}^{2}$. The associated force acting for 2 psec changes the electrons' velocity by a negligible amount $\left(<10^{-3}\right.$ $\mu \mathrm{m} / \mathrm{psec}$ ), so it will be neglected in the following equations.

\section{B. Ballistic electrons}

When the electrons are first released from the ions, there is no macroscopic electric field because the average charge density is zero. As the electrons are redistributed, the electric field grows and starts to affect the electron movement. An approximation to the initial electron movement ignores the effect of the resulting electric field. $N_{e}(\vec{r}, t)$ in Equation (3) may be expanded in a Taylor series in time. The linear term is

$$
\frac{d N_{e}(\vec{r}, t)}{d t}=\int d^{3} \vec{v} \frac{d}{d t} f(\vec{r}, \vec{v}, t) .
$$

If the electrons do not experience any forces or collisions, then equation

(2) predicts a simple evolution of the Boltzmann distribution:

$$
f(\vec{r}, \vec{v}, t)=f \vec{r}-\vec{v} t, \vec{v}, 0) \text {. }
$$

Then Equation (7) can be evaluated

$$
\begin{aligned}
\frac{d N_{e}(\vec{r}, t)}{d t} & =\int d^{3} \vec{v} \frac{d}{d t} f(\vec{r}-\vec{v} t, \vec{v}, 0) \\
& \left.=\int d^{3} \vec{v}\left(\vec{\nabla}_{r} f(\vec{r}, \vec{v}, 0) \frac{d}{d t} \overrightarrow{(r}-\vec{v} t\right)+\vec{\nabla}_{v} f(\vec{r}, \vec{v}, 0) \frac{d}{d t}(\vec{v})\right) \\
& =\int d^{3} \vec{v} \vec{v} \cdot \vec{\nabla}_{r} f(\vec{r}, \vec{v}, 9) \\
& =0 .
\end{aligned}
$$

The result is zero because $f(\vec{r}, \vec{v}, 0)$ and $\vec{\nabla}_{r}$ are isotropic in $\vec{v}$. The next term in the Taylor series involves $\vec{v} \cdot \vec{v}$, so it does not integrate to zero. Our monovelocity distribution can be easily integrated to give

$$
\Delta N_{e}(\vec{r}, t)=\frac{1}{6} t^{2} v_{0}^{2} \nabla^{2} N_{e}(\vec{r}, 0)+\ldots
$$


Other isotropic distributions of velocity would have a different coefficient, but would still redistribute as $\mathbf{t}^{2}$.

\section{Diffusive electrons}

The initial electron redistribution changes its character after the electrons start colliding with atoms. They collide after a mean time $T_{c}=\frac{L}{v_{0}}$, where $L$ is the mean free path of the electrons. The collisions are very nearly elastic because $m_{e}<<m_{a t o m}$ and it would require more than 1000 collisions to reduce the electron's energy by $5 \%$. At 100 Torr Ar, the electron undergoes 100 collisions in $1 \mathrm{nsec}$, so it is assumed the collisions are completely elastic. They only change the direction of the electron's velocity vector.

Once the electrons undergo collisions, their transport will be diffusive instead of ballistic, and they will redistribute according to Fick's Law:

$$
\frac{d N_{e}(\vec{r}, t)}{d t}=D \nabla^{2} N_{e}(\vec{r}, t),
$$

where $D=\frac{v_{0} L}{3}$ is the diffusion coefficient. The effect of the growing electric field will be introduced below.

Their mean free path is determined by the densities of their collision partners $\operatorname{Ar}\left(\sigma=3 \AA^{2}\right.$ at $2 \mathrm{eV}, \mathrm{N}=35 \times 10^{16} \mathrm{~cm}^{-3}$ at $\left.10 \mathrm{Torr}, \mathrm{L}_{\mathrm{Ar}}=100 \mu \mathrm{m}\right){ }^{2}$ $K\left(\sigma=200 \AA^{2}, N=0.8 \times 10^{16} \mathrm{~cm}^{-3}\right.$ at $\left.320^{\circ} \mathrm{C}, \mathrm{L}_{\mathrm{K}}=60 \mu \mathrm{m}\right){ }^{3}$ and other electrons $\left(\sigma=15,000 \AA^{2}\right.$ to get deflections $>0.1$ radian, $N<10^{12} \mathrm{~cm}^{-3}$, $L_{e}>6 \mathrm{~mm}$ ). These can be combined into a single mean free path by

$$
\frac{1}{\mathrm{~L}}=\frac{1}{\mathrm{~L}_{\mathrm{Ar}}}+\frac{1}{\mathrm{~L}_{\mathrm{K}}}+\frac{1}{\mathrm{~L}_{e}} \text {. }
$$

$L$ and $T_{C}$ are shown in Figure 1 as a function of $A r$ pressure. They have equal values in units of $\mu \mathrm{m}$ and psec, respectively, since the electron velocity is $1 \mu \mathrm{m} / \mathrm{psec}$. $D$ is $1 / 3$ this value in units of $\mu \mathrm{m}^{2} / \mathrm{psec}$. For all of our experimental conditions, $T_{c}<T_{2}$, the decay time of the second-harmonic 
coherence. Since the experiment integrates signal over $T_{2}$, the dynamics in a pump-probe experiment will be dominated by the diffusive regime of electron motion.

It is interesting to note that the constant slope in the diffusive regime matches that of the ballistic transport exactly at the collision time $T_{c}$. A simple connection between ballistic and diffusive transport is therefore

$$
\Delta N_{e}(r, t)=\left\{\begin{array}{lr}
\frac{1}{6} v_{0}^{2} \nabla^{2} N_{e}(r, t) t^{2} & t<T_{c} \\
\frac{1}{6} v_{0}^{2} \nabla^{2} N_{e}(r, t)\left(2 T_{c} t-T_{c}{ }^{2}\right) & t>T_{c}
\end{array}\right.
$$

Examples of the evolution of $\Delta \mathrm{N}_{\mathrm{e}}(\mathrm{r}, \mathrm{t})$ with these simplifying assumptions are presented in Figure 2. The resulting electric field will have the same time dependence. Note that increasing the Ar pressure severely dampens the evolution of $\Delta \mathrm{N}_{\mathrm{e}}$.

\section{Effects of Edc}

The complete description of electron movement and distribution must include the effects of the dc electric field that is created by their separation from the ions. The movement is known as ambipolar diffusion. The electric field can be included in the diffusive case above by considering the total chemical potential of the electrons. The non-thermal velocity distribution of the electrons will be approximated as a thermal distribution whose average speed equals the average electron speed. The total chemical potential contains contributions from the electric potential (which will be low near the beam center) and the electron density (which will be high near the beam center): 4

$$
\mu(r)=k T \ln \left(\frac{N_{e}(r)}{N_{4}}\right)-e V(r) .
$$

$V(r)$ is the electric potential and 


$$
\mathrm{N}_{\mathrm{q}}=\left(\frac{\mathrm{m} \mathrm{kT}}{2 \pi \mathrm{hbar}^{2}}\right)=1.1 \times 10^{22} \mathrm{~cm}^{-3}
$$

is the quantum density that determines where the electron gas stops behaving classically. The electron number current density (in units of $\# / \mathrm{cm}^{2} / \mathrm{sec}$ ) is given by

$$
\begin{gathered}
\vec{J}_{\mathrm{e}}(r, t)=\frac{-D N_{\mathrm{e}}}{k T} \vec{\nabla} \mu(r, t) \\
=-D \mathrm{DN}_{\mathrm{e}}(r, t)-\frac{e D N_{\mathrm{e}}(r, t)}{k T} E_{d c} .
\end{gathered}
$$

From $\mathrm{J}_{e}$, the evolution of $\mathrm{N}_{\mathrm{e}}$ is determined from the continuity equation,

$$
\frac{\partial N_{e}(r, t)}{\partial t}=-\vec{\nabla} \cdot \vec{J}_{e}(r, t) \text {. }
$$

Because $E_{d c}$ involves an integral of $N_{e}(r, t)$, this set of equations cannot be worked out analytically. However, it can be treated numerically on a computer. The resulting radially symmetric electric field is always zero at the origin and a maximum at approximately the radius of the original electron distribution. The magnitude of the maximum field is shown in Figure 3 as a function of time for an ion cloud radius of $100 \mu \mathrm{m}$ and five different central ion densities ranging from $10^{10}$ to $10^{12}$ ions $/ \mathrm{cm}^{3}$. The ionization radius is smaller than the beam radius because of the nonlinear dependence of ionization on intensity. Figure 3 shows that the electric field initially grows linearly in time, but then saivrates. The saturation occurs when the two terms contributing to $\mathrm{J}$ in Equation (16) balance and cancel each other at all points in space. The system is then in a quasi-equilibrium, and the charge distribution will not change until the electrons cool many nanoseconds later. The ions will also experience this field, but even after one microsecond they will have only moved a few microns.

The electric field does not overshoot its equilibrium value and therefore does not ring or oscillate. This is implicit in the assumption of 
diffusive behavior in Equation (16). Physically, the electrons are undergoing collisions every few tens of picoseconds, so they cannot overshoot their equilibrium position on any longer time scale.

The actual density of ions and electrons released in the experiment can be estimated from the ionization rate of atoms excited to the $9 \mathrm{~d}$ state. The cross section for ionization is $3.7 \times 10^{-20} \mathrm{~cm}^{2,5}$ so the 3 picosecond shot of $10^{10} \mathrm{~W} / \mathrm{cm}^{2}$ light will produce about $2 \times 10^{11}$ ions $/ \mathrm{cm}^{3}$, closest to the second curve from the top in Figure 3 (corresponding to $3 \times 10^{11}$ ions $/ \mathrm{cm}^{3}$ ). The intensities used are not well-measured because of the irregularity of the beam profile, so the ionization may be significantly different due to its $\mathrm{I}^{3}$ dependence. The electric field is saturating within a couple hundred picoseconds, so in order to observe the changing field, the experiment needs time resolution of less than $100 \mathrm{psec}$. Most of our observations can be understood with the assumption that the electric field saturates before the coherence has significantly decayed.

Curves of the final electron distributions for several central ion densities are shown in Figure 4a. At high central densities, the equilibrium distribution is very close to that of the ions, while at low central densities, it is significantly different. Equilibrium electric fields as a function of $r$ are shown in Figure $4 b$.

Because the electric field is proportional to the integrated difference between $\mathrm{N}_{e}$ and $\mathrm{N}_{\mathrm{i}}$, the maximum electric field is seen to saturate as $\mathrm{N}_{\mathrm{i}}$ is increased (Figure 5). This result differs strikingly from Bethune's estimate, ${ }^{1}$ which calculates the maximum electric field by equating the static field energy with the total initial kinetic energy of the electrons. Since total energy must be conserved, such an equation would require all electrons to come to a rest 
simultaneously so their kinetic energy is zero. This is clearly unphysical. The above equations describe a diffusing electron gas whose temperature determines its distribution in a shallow potential well.

For the case of the nanosecond experiments done previously, a rough estimate of the iơnization results in $>10^{13}$ ions $/ \mathrm{cm}^{3}$. Indeed, Okada et al., observed a saturation of the ionization, ${ }^{6}$ indicating that most of the atoms in their focal volume were being ionized. They concluded that they produced $10^{16}$ ions $/ \mathrm{cm}^{3}$. In fact, $10^{13}$ ions $/ \mathrm{cm}^{3}$ are enough to create an equilibrium electric field independent of the ion density. Thus it is not surprising that many nanosecond experiments did not generate greater SHG when they created greater numbers of ions.

What does change with $\mathrm{N}_{0}$ is how far the electrons must travel to reach equilibrium, and thus the time scale of the changing electric field. This will be an important factor in determining the parameters of pump-probe experiments used to verify this model.

\section{Experimental Set-up}

These experiments are done using a tunable amplified picosecond laser pulse. A continuous-wave mode-locked YLF laser (13 Watts, $100 \mathrm{MHz}$, 80 psec puises, $1.054 \mu \mathrm{m}$ wavelength) is doubled to $700-1000 \mathrm{~mW}$ of $527 \mathrm{~nm}$ light in a temperature-tuned, noncritically phase-matched LBO crystal. The green light synchronously pumps a mode-locked dye laser operating with Rhodamine $6 \mathrm{G}$ dye, yielding $50-100 \mathrm{~mW}$ of $570-620 \mathrm{~nm}$ light in 4 psec pulses. These pulses are amplified by four stages of Bethune cells pumped at $10 \mathrm{~Hz}$ by a $20 \mathrm{nsec}$ Q-switched YAG laser. The beam is focused through a saturable dye 
jet at two points in the chain to eliminate amplified spontaneous emission(ASE). The ASE is held to below $1 \mu \mathrm{J} /$ pulse.

The result is $\approx 200 \mu \mathrm{J}$ laser pulses that have a poor transverse mode quality due to severe gain saturation in the center of the amplifying cells. Figure 6 shows a CCD camera picture of the original dye beam after passing through all of the amplifier stages without being pumped, and Figure 7 shows the same beam with amplification. The horizontal scales are identical, so the much larger beam radius after amplification shows that the beam edges have undergone many more factors of amplification than the beam center. The pulse duration is 3 psec, as shown by the collinear autocorrelation curve in Figure 8.

The pulse energy also varied significantly from pulse to pulse due to variable pump laser intensities. Since the pump intensity determines the exponential gain seen in the amplifiers, the final pulse energy is highly sensitive to it. The sensitivity is weakened if the laser pulse reaches saturation (where all excited molecules in the beam path are stimulated to emit their stored energy), but this only occurs in the beam center. The edges of the beam, still undergo exponential growth in the final amplifier stage, so the total beam energy is sensitive to the pump intensity.

The pump laser's energy integrated over its 20 sac duration remains constant to within a couple per cent. However, the amplifier dye has a lifetime of only $1 \mathrm{nsec}$, so the amplification is sensitive to shorter-time-scale variations. Using an oscilloscope with $600 \mathrm{MHz}$ bandwidth, we observe oscillations at $500 \mathrm{MHz}$ of about $25 \%$ of the average laser intensity. There could easily be additional structure that is too fast for the scope to reproduce. 
The oscillations shift with each pulse, so the useful pump intensity varies by at least $25 \%$, and the amplified laser energy varies by much more.

The acquired data was sorted according to the energy measured for each pulse. Typically, we used five to ten bins with each bin covering about $10 \%$ of the average pulse energy. Thus the input energies are known to within 5\%, even though the laser energy was less well-controlled.

To do pump-probe experiments, the laser was split by a $50 / 50$ dielectric coating beamsplitter on a $1 \mathrm{~mm}$ glass substrate. The layout of the experiment is shown in Figure 9. The beams were sent off-axis through metallic retroreflectors accurate to 5 arcseconds and recombined at a second beamsplitter. The position and tilt of the pump beam could be controlled independently of the probe beam. Vibrations moved the retroreflectors by more than a wavelength, so at positions of overlap, the laser intensity fluctuated randomly between constructive and destructive interference. The multi-shot averaging smoothes the interference fringes present in a collinear autocorrelation. The beams were then focused by a $+15 \mathrm{~cm} /-5 \mathrm{~cm}$ lens pair to a spot size of $80-400 \mu \mathrm{m}$, depending on the distance between lenses.

The heat pipe is $45 \mathrm{~cm}$ long with a $15 \mathrm{~cm}$ zone heated by a $500 \mathrm{~W}$ resistive heat tape and insulated with 4-inch fiberglass insulation. The heat tape was powered by a Variac to control the oven temperature. Windows on each end allowed the passage of the laser light. Cooling water circulated in external pipes wrapped around the oven $15 \mathrm{~cm}$ from the oven center. The outside temperature of the heat pipe was measured with a thermocouple. It was calibrated with the inside temperature by replacing one window with a coupler to a $0.6 \mathrm{~cm}$ diameter, thin-walled stainless steel tube, inside of which 
was placed a thermocouple. Internal temperatures were typically $10^{\circ}$ below the measured outside temperature.

The $3 / 4$ meter $f / 7$ double monochromator had an adjustable resolution. The $9 \mathrm{~d}-11 \mathrm{~s}$ spectral distance is $3 \AA$, while the distance to the $10^{4}$ stronger $10 \mathrm{p}$ light is $15 \AA$, so the resolution used was typically $1 \AA$. The beam was focused into the first monochromator slit with a $5 \mathrm{~cm}$ quartz lens, giving an acceptance area of $(7 \mathrm{~mm})^{2}$ and an acceptance angle of $2 \mathrm{mrad}$. A head-on PMT close-coupled to the monocitromator output assured uniform detection. The total efficiency at the UV wavelength was calculated to be $1 \%$ and verified at visible wavelengths. The data is given in units of observed counts/shot and should be multiplied by 100 to obtain actual photons/shot generated by the vapor.

A window before the oven split off two $4 \%$ beams for reference purposes. One was doubled in a phase-matched LBO crystal that was placed at the position in the ghost beam equivalent to the oven center in the main beam. This nonlinear reference arm assured overlap in autocorrelation measurements and reflected changes in beam profile or duration. The reference SHG was easily measured with a UV sensitive photodiode.

\section{Results}

The spectral character of the generated light is demonstrated by a monochromator scan of the region $2945-3015 \AA$. This covers the $9 \mathrm{~d}$ resonance (used for almost all other measurements), its close neighbor, 11s, and the p, s, and $\mathrm{d}$ states on either side of this pair (Figures 10 and 11). Because of the wide dynamic range of measurement, Figure 10 uses a logarithmic vertical scale, and it is clear that the second-harmonic light at $2974 \AA$ is $30 x$ higher than the 
background. The strongest light at 2989 , the $10 \mathrm{p}$ resonance, is assumed to be allowed four-wave-mixing amplification of spontaneous emission. It has not been measured any more closely than its relative peak height. The light from the $11 p$ resonance is surprising because it is at a higher energy than the $2 \omega$ resonance. It may be due to allowed four-wave $\left(\chi^{(3)}\right)$ mixing of two $\omega$ photons and an infrared photon generated by the four-wave-mixing with the 10p state, or by the $\chi^{(5)}$ process described in Chapter 3.

The distinct signal at the far-off, unallowed transitions $8 \mathrm{~d}$ and 10 s has not been explained. It may well be related to the second-harmonic in that an electric field which allows the coherent $9 \mathrm{~d}$ population to radiate would also allow a coherent $8 \mathrm{~d}$ population to radiate. It is unclear how these nonresonant states would obtain a coherent population. The dc electric field will cause some coherent transfer to the $8 \mathrm{~d}$ state, and even the ac light field might induce a population because it is very strong compared to the atomic potential of the $9 \mathrm{~d}$ state. However, even if the coherent $8 \mathrm{~d}$ population equaled that of the $9 \mathrm{~d}$, the phase mismatch with the produced light beam would be orders of magnitude greater. These emissions certainly merit greater attention in the future.

The resonant nature of the SHG is demonstrated in Figure 12, where the laser frequency is scanned while keeping the monochromator frequency fixed at the $9 \mathrm{~d}$ output wavelength. Similar scans with the monochromator fixed off-resonance showed no signal. We also see signal at the $11 \mathrm{~s}$ resonance, which is expected in the ionization model because both resonances are twophoton allowed and will enhance ionization, and both may be mixed with pstates by an electric field in order to radiate. 
The coherent nature of the SH light is verified by two experiments: the collimated nature of the output beam and the observation of phase matching. We measured the output structure by scanning a $100 \mu \mathrm{m}$ slit across the beam. A vertical slit scanned horizontally gave the single sharp peak in Figure 13a. By comparing with the simultaneously measured laser structure in Figure 13b, it is clear that the SHG is even more collimated than the laser. Also, it is strongly peaked at the point of maximum laser intensity. A horizontal slit scanned vertically (shown in Figure 14 a\&b) gives a strikingly different result. in this direction, the laser is much narrower, and the SHG is broader and split into two lobes, although it is still a collimated beam. The information from these scans can be combined into the three-dimensional surface plots in Figure 15 by assuming that each profile is independent of the orthogonal direction. Although crude at best, the graphs show that the SHG is generated on the steepest slopes of the laser light's intensity profile. The input laser is vertically polarized by a factor of $10^{5}: 1$, and the output polarization was measured to be vertical by a factor greater than 20:1. The fact that the lobes are also oriented along the polarization direction prevents us from concluding whether it is the gradients or the polarization that determine their orientation. An experiment with the orthogonal polarization is planned for the near future.

The phase-matching curve in Figure 16 also demonstrates the coherence of the SHG. The SHG light grows according to

$$
\frac{\partial E_{3}(z)}{\partial z}=A_{1} P_{3}(z) \exp (i \Delta k z)
$$

where $A_{1}$ is a constant, $E_{3}(z)$ is the generated electric field at $2 \omega$, and $P_{3}(z)$ is the medium's polarization at $2 \omega$. If $\Delta k$ is dominated by the potassium, it is linearly proportional to $N_{K}$, as is $P_{3}(z)$. Thus the final electric field is 


$$
E_{3}\left(z_{f}\right)=\int_{0}^{z_{f}} d z A_{1} A_{2} N(z) \exp \left(i\left(A_{3} \int_{0}^{z} d z^{\prime} N\left(z^{\prime}\right)\right)\right)
$$

Changing variables to

$$
N_{T}(z)=\int_{0}^{z} d z^{\prime} N\left(z^{\prime}\right)
$$

lets Equation (19) be integrated:

$$
E_{3}\left(z_{f}\right)=\frac{A_{1} A_{2}}{i A_{3}}\left(\exp \left(i A_{3} N_{T}\left(z_{f}\right)\right)-1\right\} \text {. }
$$

This is independent of the structure within $N(z)$. The signal should then follow a $\cos ^{2}$ curve in $N_{T}$, so the exponential dependence of $N$ on the temperature predicts an oscillatory function of $T$ with increasing frequency. In Figure 16, the signal rises from zero at $260^{\circ} \mathrm{C}$ and reaches the first phasematching peak at $315^{\circ} \mathrm{C}$ (outside the oven). Calculations from the known $\Delta \mathrm{k}$ predict the peak at $295^{\circ} \mathrm{C}$ assuming a $10 \mathrm{~cm}$ uniform vapor. The cooler temperatures inside the oven than outside, and a shorter actual heated zone may account for the difference in peak temperatures.

The minimum at $325^{\circ} \mathrm{C}$ does not reach zero, and there are two plausible explanations: 1) the signal is averaged over several minutes, so fluctuations in $\mathrm{N}_{\mathrm{T}}$ with time (due to currents inside the oven) may wash out the contrast; 2) the laser changes the index of refraction of the vapor by exciting the potassium atoms, and the changes are not uniform because the laser is not uniform. The latter explanation was favored by Mossberg ${ }^{7}$ because they observed the contrast decrease with increasing laser intensity. We cannot be more quantitative than this without more detailed measurements of our oven's temperature profile, but the phase-matching 
oscillation clearly demonstrates that SHG is a coherent mixing process between the laser field and the generated light field.

Signal resulting from the second-harmonic coherence calculated in Chapter 3 would be proportional to $1 / \mathbf{P}_{\mathrm{Ar}}$ at high pressures if the symmetrybreaking were constant and independent of $P_{A r}$. This dependence would level off at low pressures where inhomogeneous broadening would dominate. Such curve is compared with experiment in Figure 17. It is clear that the data drops more quickly than the prediction near the origin. This could occur for any symmetry-breaking mechanism that either grew with time or was weakened or slowed by the increased Ar pressure. The ionization model predicts both effects. The electric field increases initially with time, and its rate of increase depends on the Ar pressure. At higher pressures, the electrons diffuse more slowly. These effects alone predict the concaveupward curve on the same graph.

Once the dc field starts reaching equilibrium during the coherence decay, the predicted $1 / \mathrm{P}$ dependence will weaken. Since the time to reach equilibrium drops steeply with $1 / P$, it could soon be much less than the coherence decay time. Beyond this point the curve would follow the solid curve predicted by a constantly-broken symmetry. Unfortunately, the onset of saturation is strongly dependent on the density of electrons released. This density is difficult to calculate with any confidence because the beam profile is not Gaussian.

The intensity dependence of the SHG is quite strong, as shown in Figure 18, where it is plotted against the sixth power of the laser intensity. At the lower intensities, it is fairly linear, but at higher intensities, the intensity dependence falls off. Unfortunately, the ionization model predicts a variable 
intensity dependence. With low amounts of ionization, equilibrium is not approached during the decay of the coherence. The dc electric field depends linearly on the number of ionized electrons which in turn depends on the cube of the laser intensity. The second-harmonic intensity will then be proportional to $I^{8}$. At the other extreme of high ionization and fast equilibrium, the dc field is independent of intensity, so the SHG will be proportional to $R$. At even higher intensities, the laser ionizes a significant fraction of the potassium atoms. This decreases the nonlinearity of the medium and lowers the intensity dependence even further. Since none of these effects will happen suddenly, the total dependence should curve from $I^{8}$ to $I^{2}$ or below fairly gradually. The measured intensity dependence curves gradually from about $I^{6}$ to about $I^{4}$. This is a poor way to test the ionization model.

The best way to test the model is to observe the symmetry as it is being broken using a pump-probe experiment. This requires a careful selection of experimental parameters because with a constant laser energy, the signal depends very strongly on the laser beam radius (via the intensity), as dres the time needed to reach equilibrium. Two effects must then be balanced. A small radius is needed to obtain a significant or even measurable signal, while a large radius will slow the time it takes to reach equilibrium. Another critical parameter is the argon pressure. The time resolution is defined by the decay of the $2 \omega$ coherence and rises with Ar pressure, while the signal drops. An intermediate pressure must be chosen to obtain adequate signal as well as adequate time resolution.

At low pressures, the coherence time is long and the only delay dependence present is a sharp autocorrelation peak near $\tau=0$ (Figure 19). At 
high pressures (100 Torr), there is no signal for any delay up to one nanosecond. At an intermediate pressure of $\mathbf{3 0}$ Torr and with fairly tight focusing to a beam waist of about $80 \mu \mathrm{m}$, we did see the signal increased by about 3 between short delays and long delays, as shown in Figure 20. The sum of sigials from the two beams acting individually was just $\mathbf{0 . 0 5}$ counts/shot, equal to the signal with short delays. The scan is not fine enough to observe an autocorrelation peak near $\tau=0$. The signal rises with delay up to about $100 \mathrm{psec}$ and then saturates. The data is not clean enough to distinguish anything of the shape of the rise. Better measurements in the near future will allow a more quantitative assessment of the ionization model.

\section{v. Conclusion}

The measurements so far prove that the SHG from a picosecond laser pulse is the result of a coherent mixing of the laser light. It is qualitatively consistent with the ionization model of vapor SHG. In evaluating the model, it is important to include the effects of a slowly decaying second-harmonic coherence as well as a saturating dc electric field. Continued work on this project will afford us more quantitative results and a more detailed analysis. A fuller understanding will lead us to a better picture of a laser's complex interaction with a simple vapor, and to tantalizing questions about the other forbidden light emitted by this surprisingiy complicated system. 


\section{References}

1. D. S. Bethune, Phys. Rev. A 23 (6): 3139 (1981).

2. D. E. Golden and H. W. Bandel, Physical Review 149 (1): 58 (1966).

3. A. Hasdan, T. M. Miller and B. Bederson, Phys. Rev. A 8 (3): 1563 (1973).

4. C. Kittel and $\mathbf{H}$. Kroemer, Thermal Physies (W. H. Freeman, New York, 1980).

5. D. R. Bates, Atomic and Molecular Processes (Academic Press, New York, 1962).

6. J. Okada, Y. Fukuda and M. Matsuoka, J. Phys. Soc. Jap. 50 (4): 1301 (1981).

7. T. Mossberg, A. Flusberg and S. R. Hartmann, Opt. Commun. 25 (1): 121 (1978). 

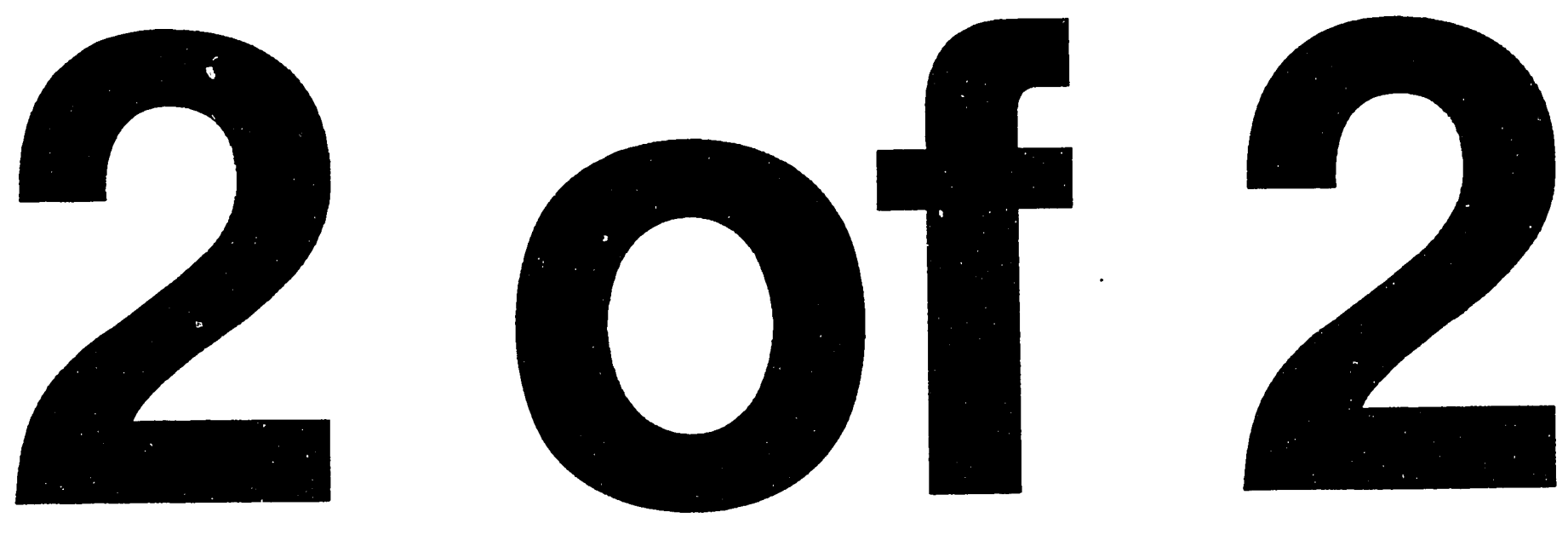


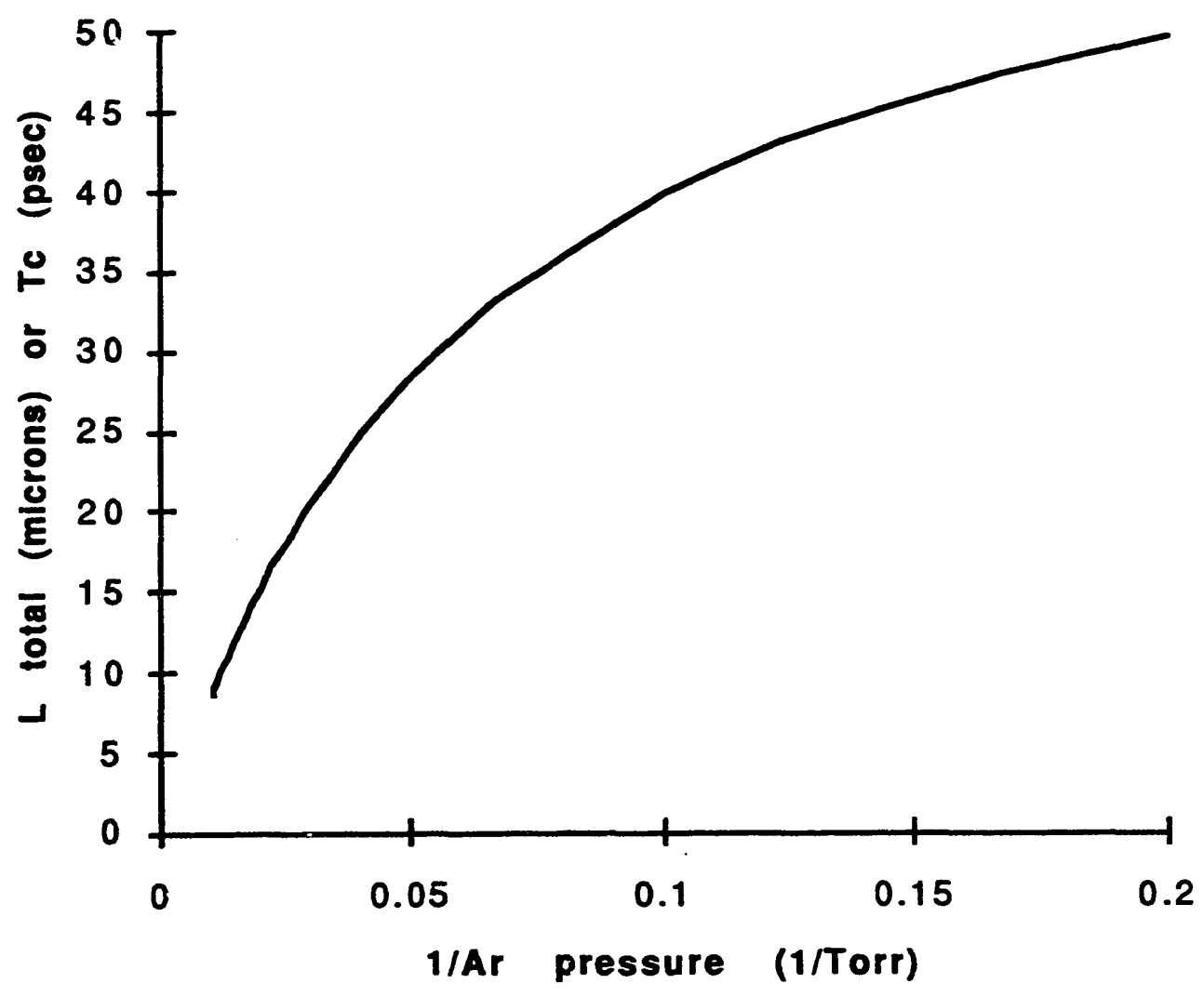

Figure 1: $\quad$ Mean free path (Ltotal) and mean time between collisions $\left(T_{c}\right)$ (both shown as _-, since they have equal numerical values) of electrons as a function of the inverse argon pressure. The diffusion constant, $D$, is numerically $1 / 3$ of these values. 


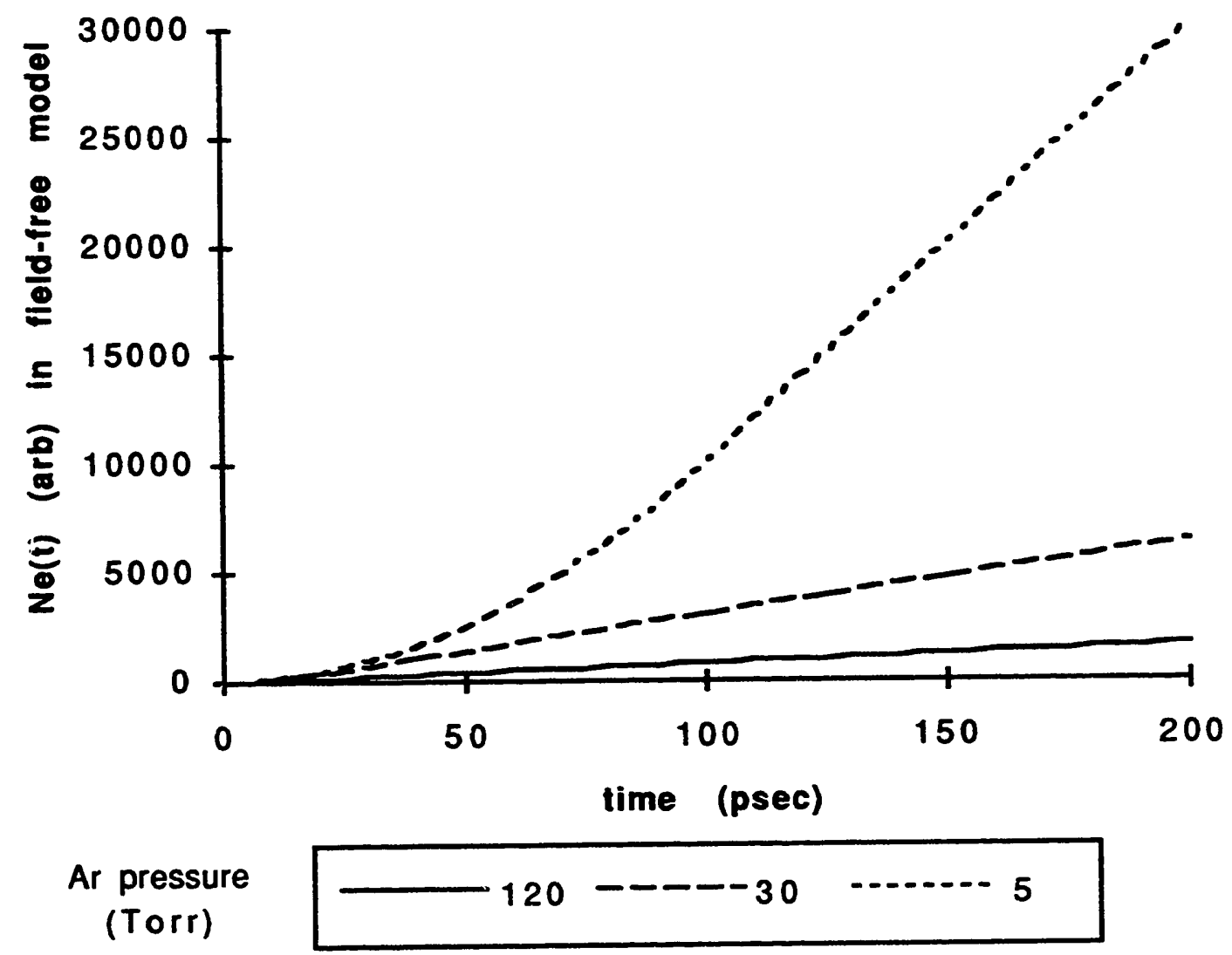

Figure 2: $\quad \mathrm{N}_{\mathrm{e}}(\mathrm{t})$ for the interpolated ballistic-diffusive model, assuming different mean free paths, for different argon pressures. 


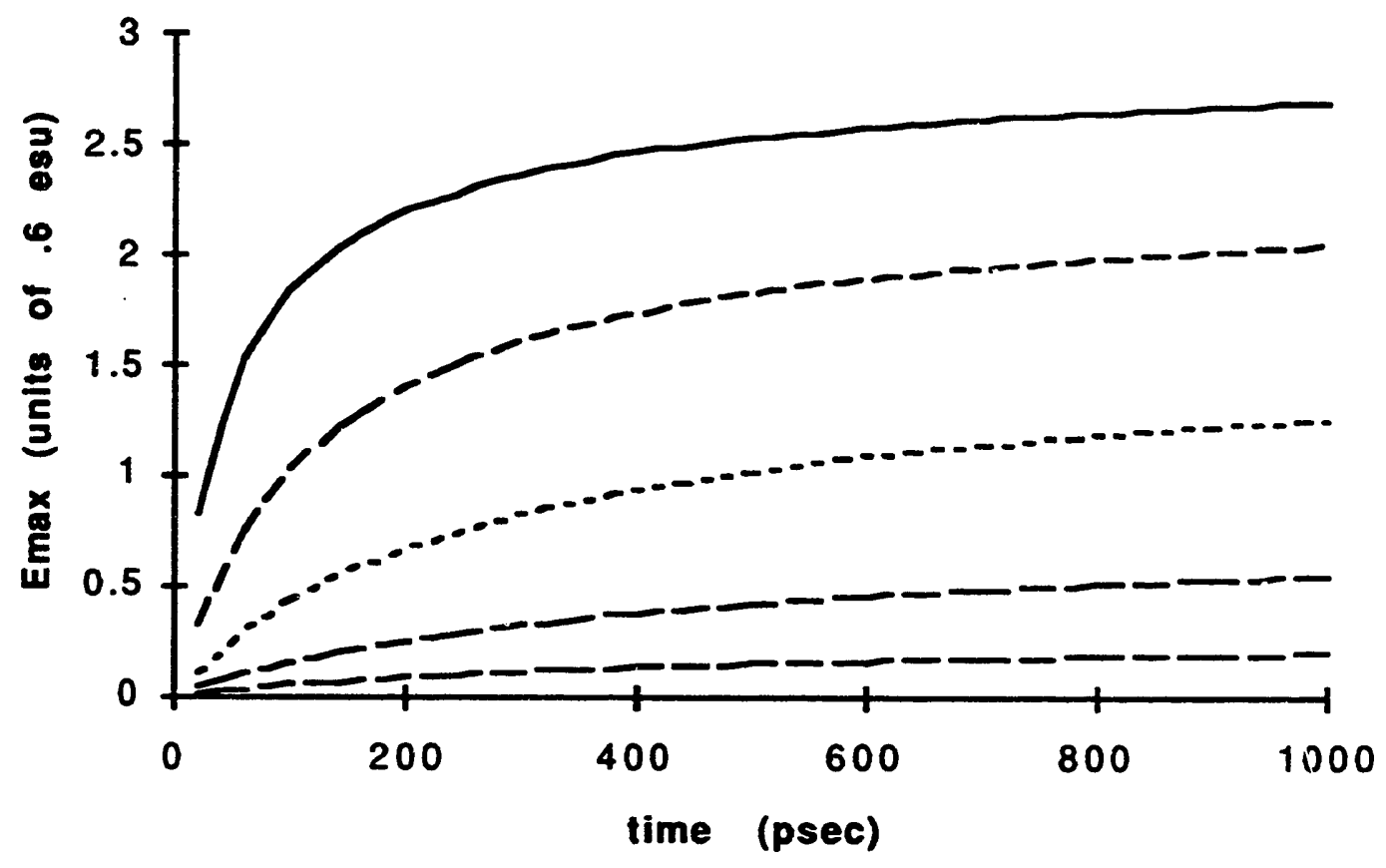

Figure 3 - Numerical results from ambipolar diffusion: maximum electric field as a function of time for five different central ion densities assuming an ion radius of $100 \mu \mathrm{m}$, ranging from $10^{10}$ to $10^{12}$ electrons $/ \mathrm{cm}^{3}$. The initial development is linear as predictiad by the diffusive part of Equation (13). 


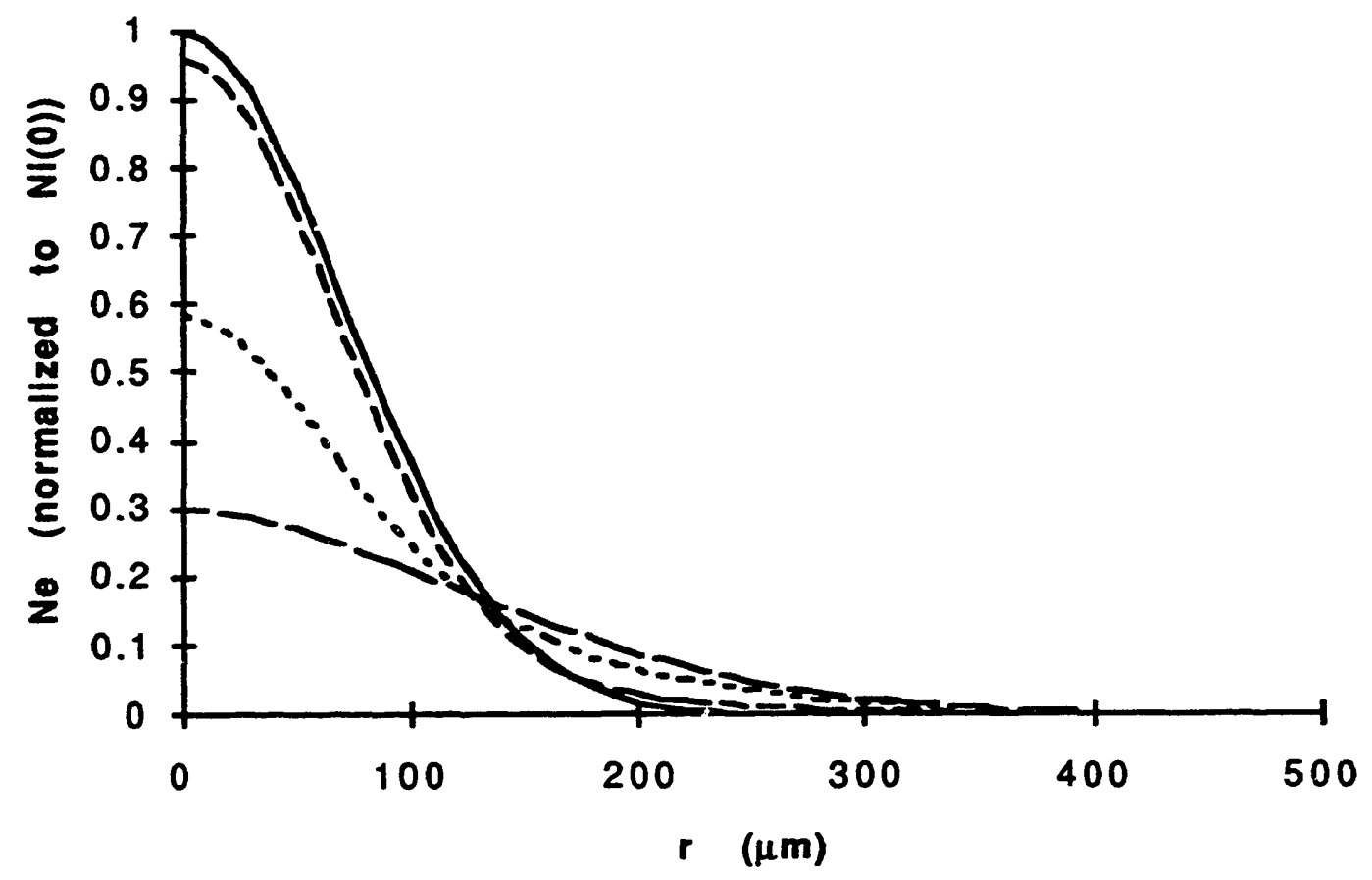

Figure 4a: Equilibrium electron distributions normalized to their 3 central ion densities of $10^{10}, 10^{11}$, and $10^{12} / \mathrm{cm}^{3}$. The higher the density, the lesser the movement, resulting in a saturating electric field. 


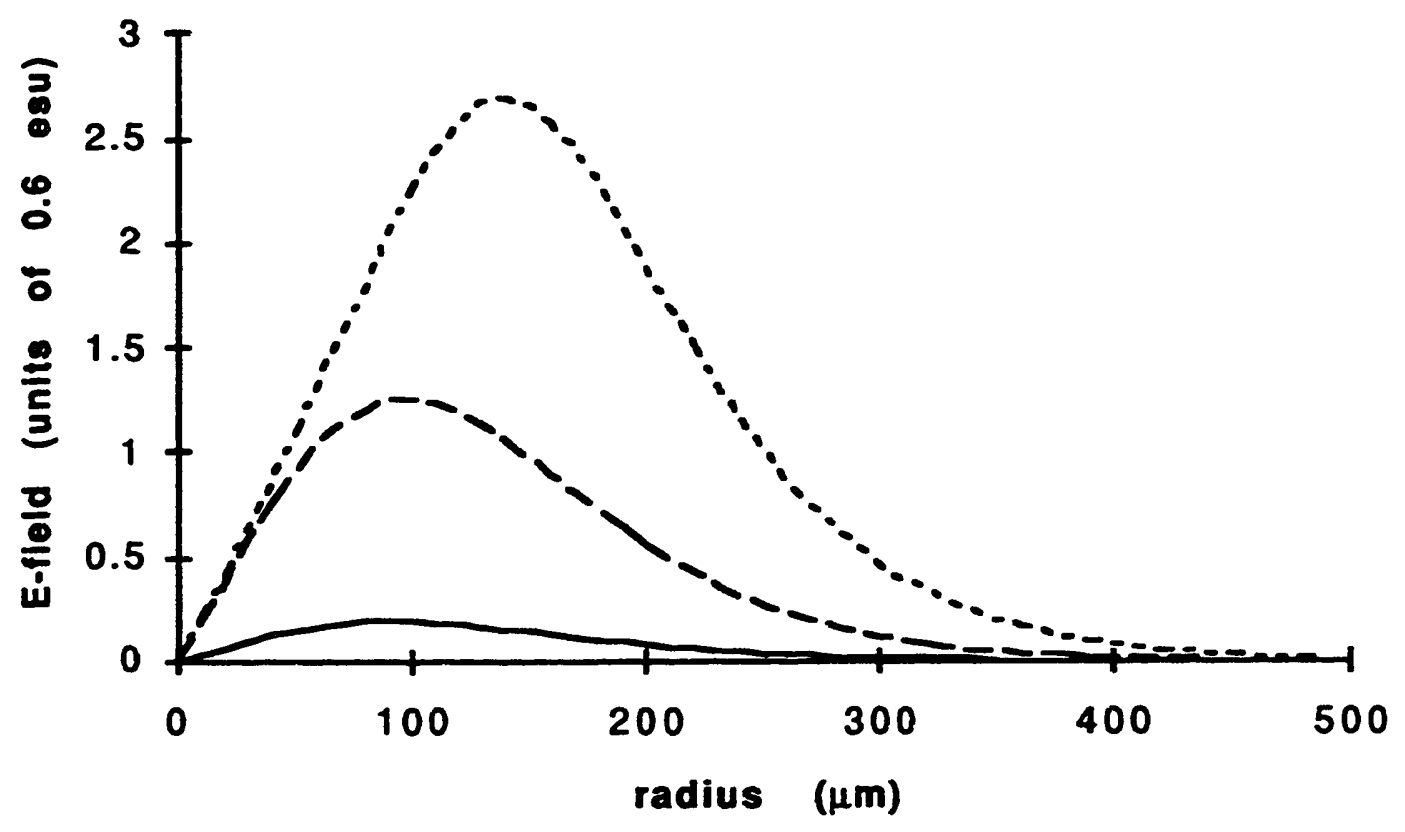

Figure $4 \mathrm{~b}$ - Equilibrium dc electric fields for 3 different central ion densities, $10^{10}, 10^{11}$, and $10^{12} / \mathrm{cm}^{3}$. The shape is fairly independent of $\mathrm{N}_{0}$, and the maximum field is seen to saturate, since the second factor of ten increase in ion density resulted in only a factor of two increase in field. 


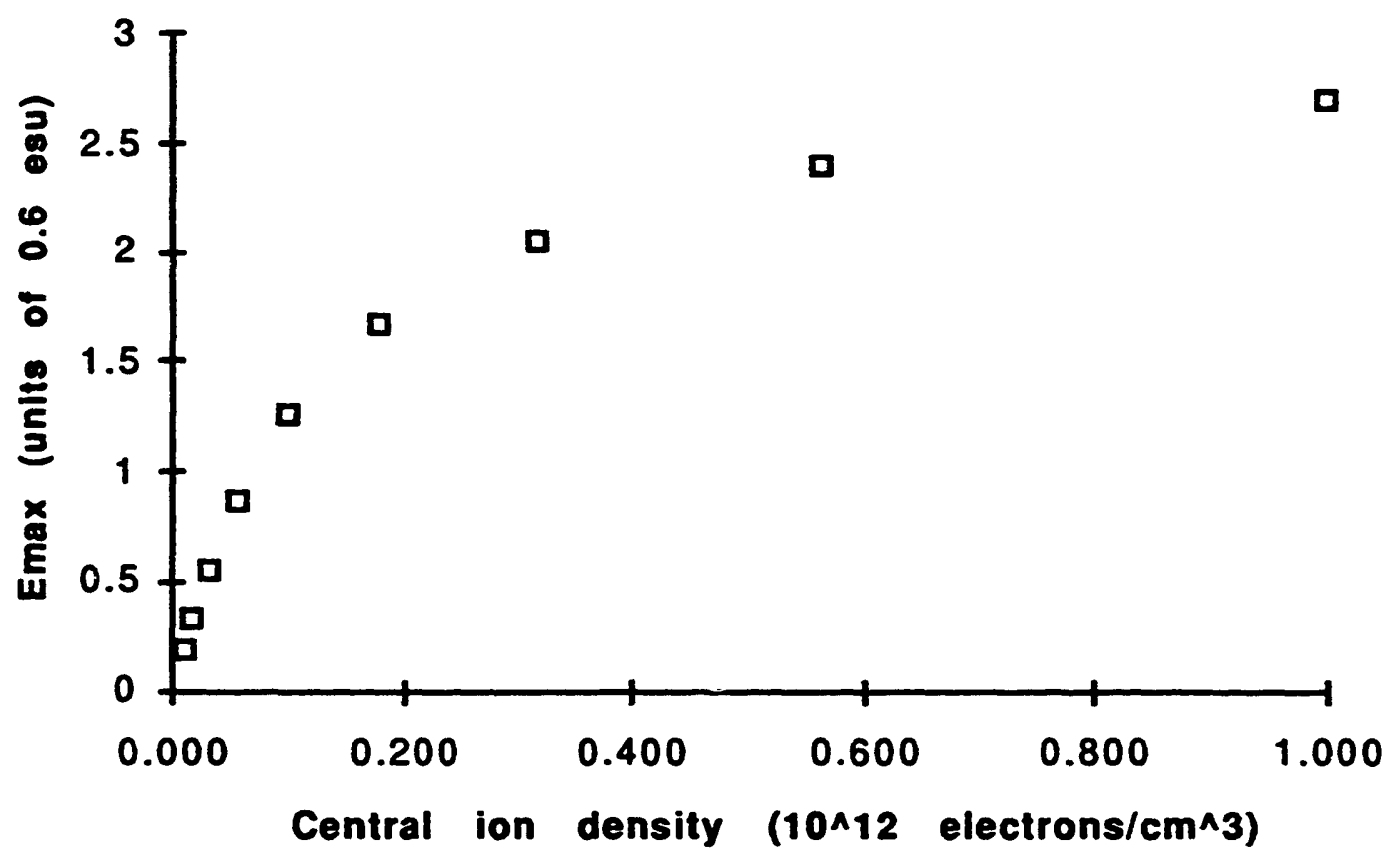

Figure 5 - Saturating maximum electric field as a function of central ion density. 
Figure 6 - CCD picture of the laser beam before amplification. 


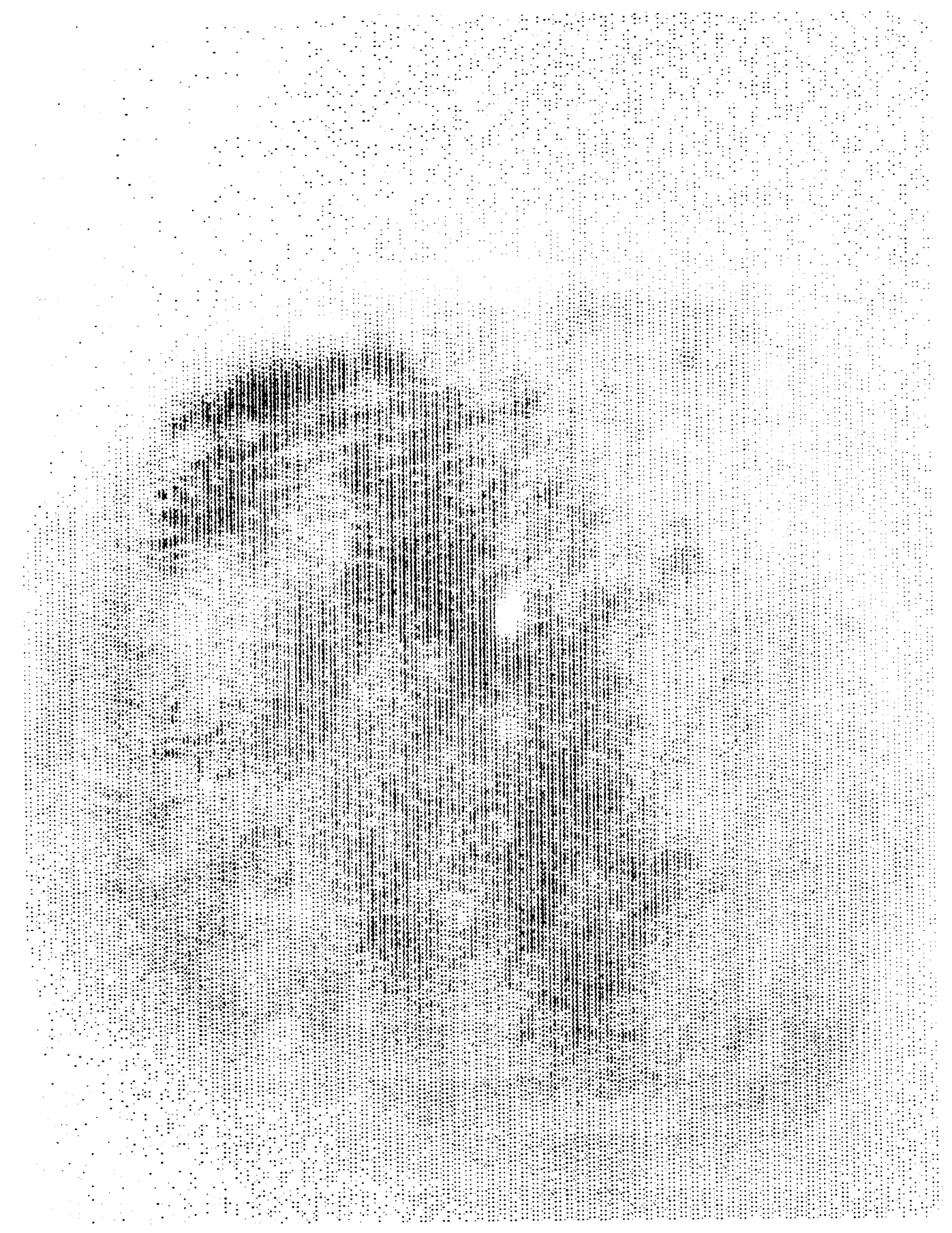

Figure $7-C C D$ picture of the laser beam after amplification, using the same scale as Figure 6. 


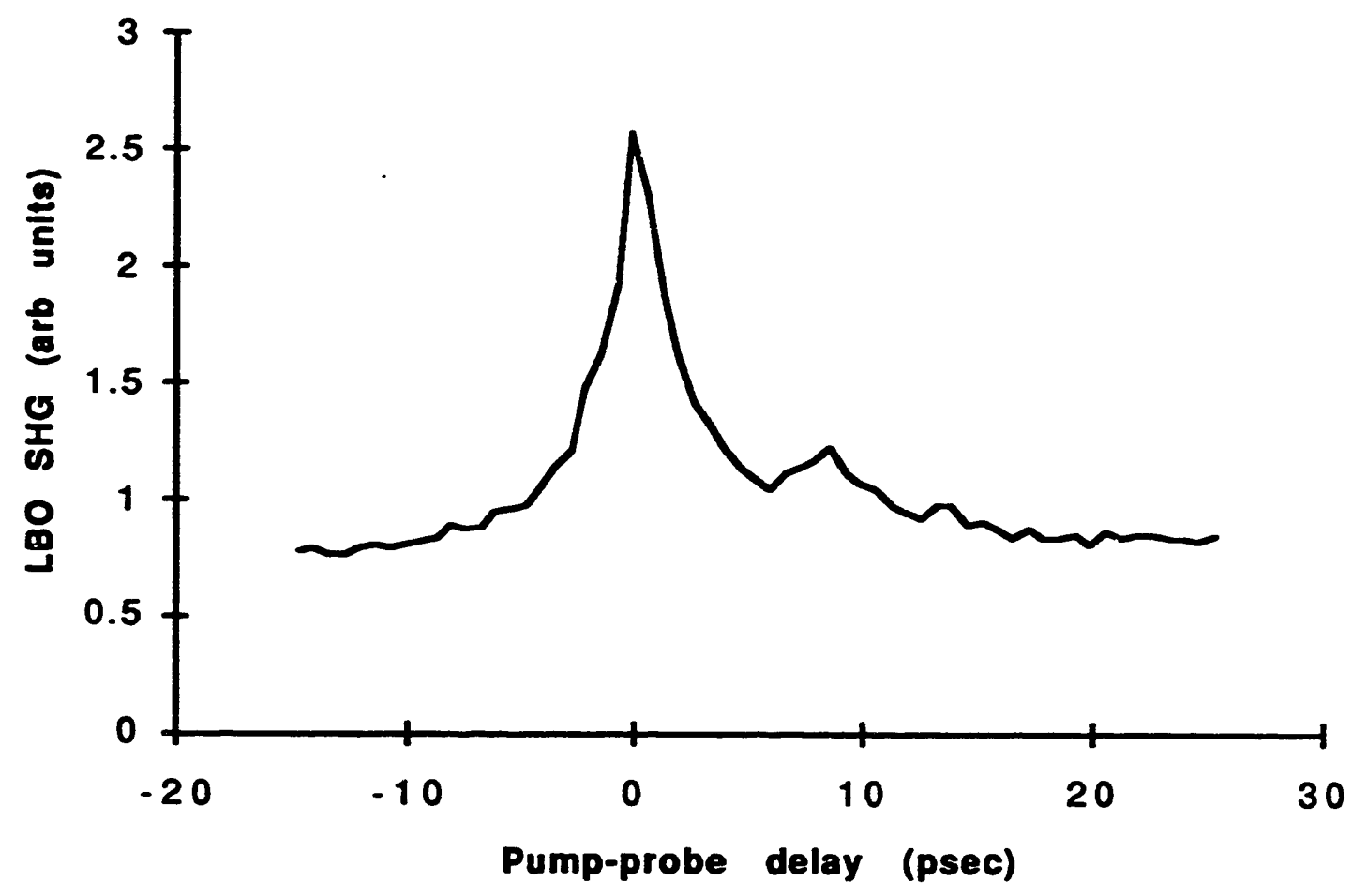

Figure 8 - Collinear autocorrelation trace in LBO. Signal averaging smoothes out the interference fringes, and $\mathrm{R}$ intensity dependence predicts a contrast of 3:1 between the peak signal and the signal at long delays. Pulsewidth from this measurement is $2-3$ psec. The secondary peak on the right is a ghost reflection from the beam splitter. 


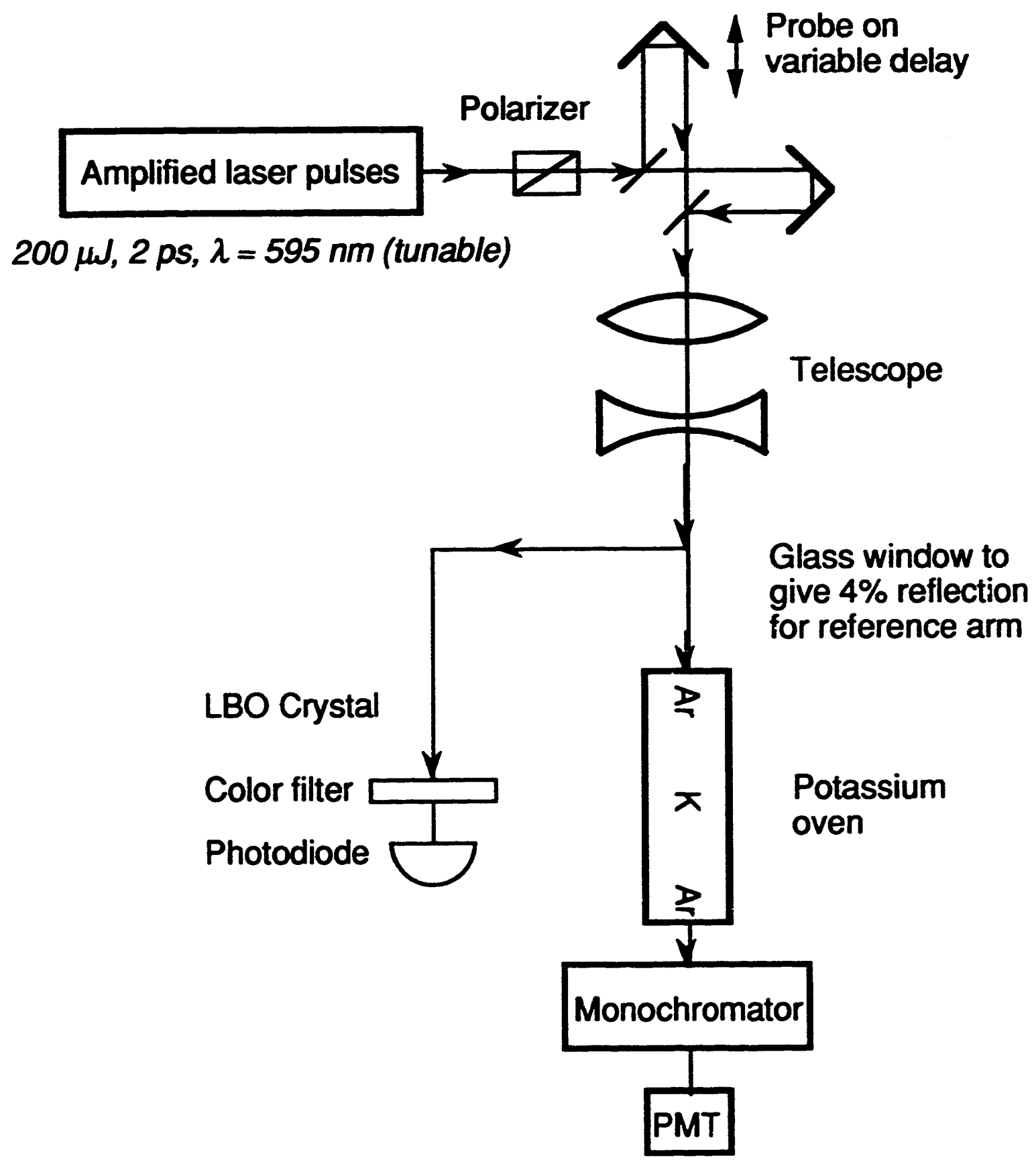

Figure 9 - Layout of a collinear pump-probe SHG experiment. 


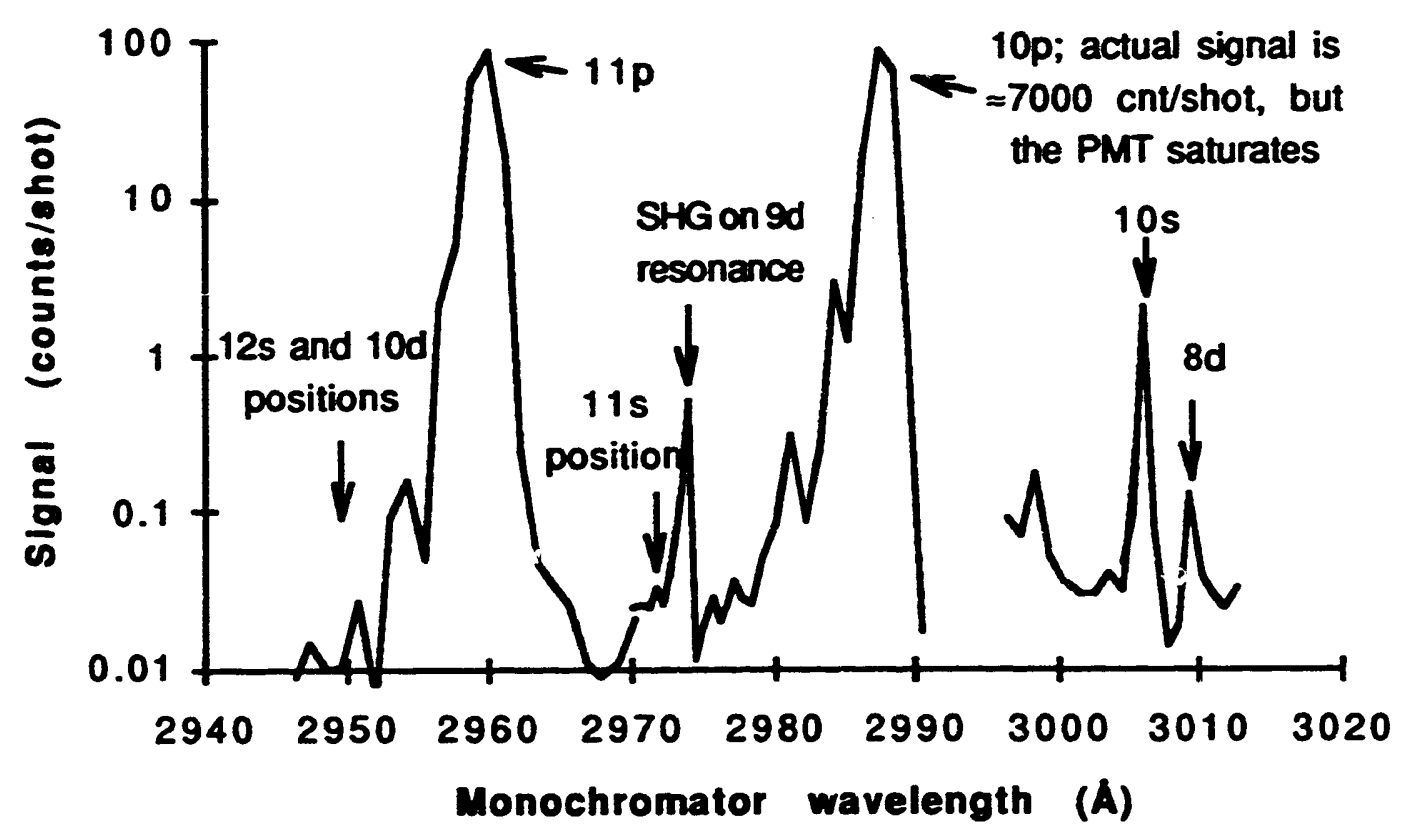

Figure 10 - Output spectrum for 9d two-photon resonance, on a logarithmic vertical scale to show the wide dynamic range. The monochromator wavelength is 4 Angstroms less than the actual vacuum wavelength. 


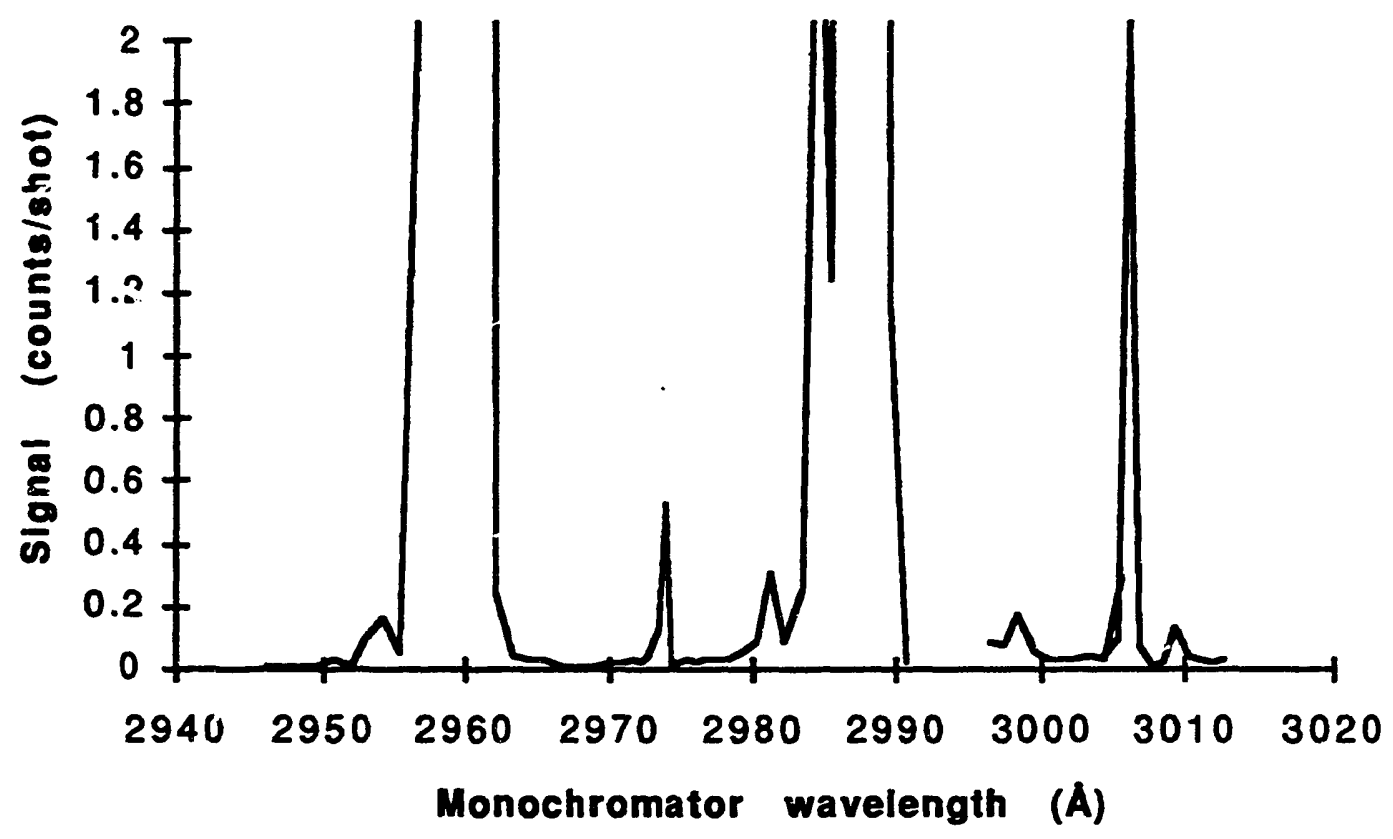

Figure 11- Same data as in Figure 10, but on a linear scale to give a better indication of the signal to noise ratio. 


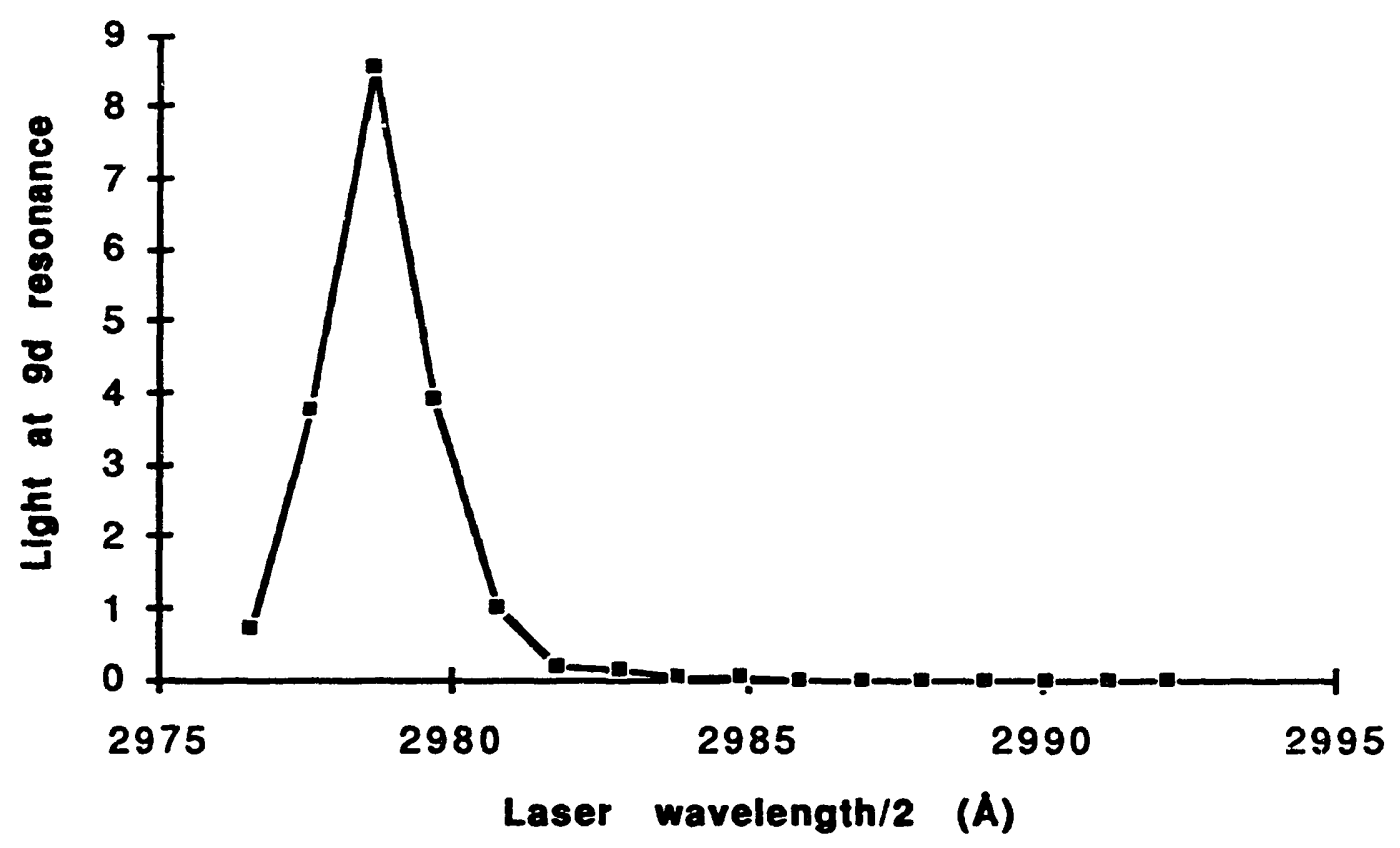

Figure 12 - Light detected at 2979 Angstroms (2975 on the monochromator) as a function of the laser tuning near the $9 \mathrm{~d}$ resonance. Similar scans detecting light at off-resonant frequencies saw no signal. 

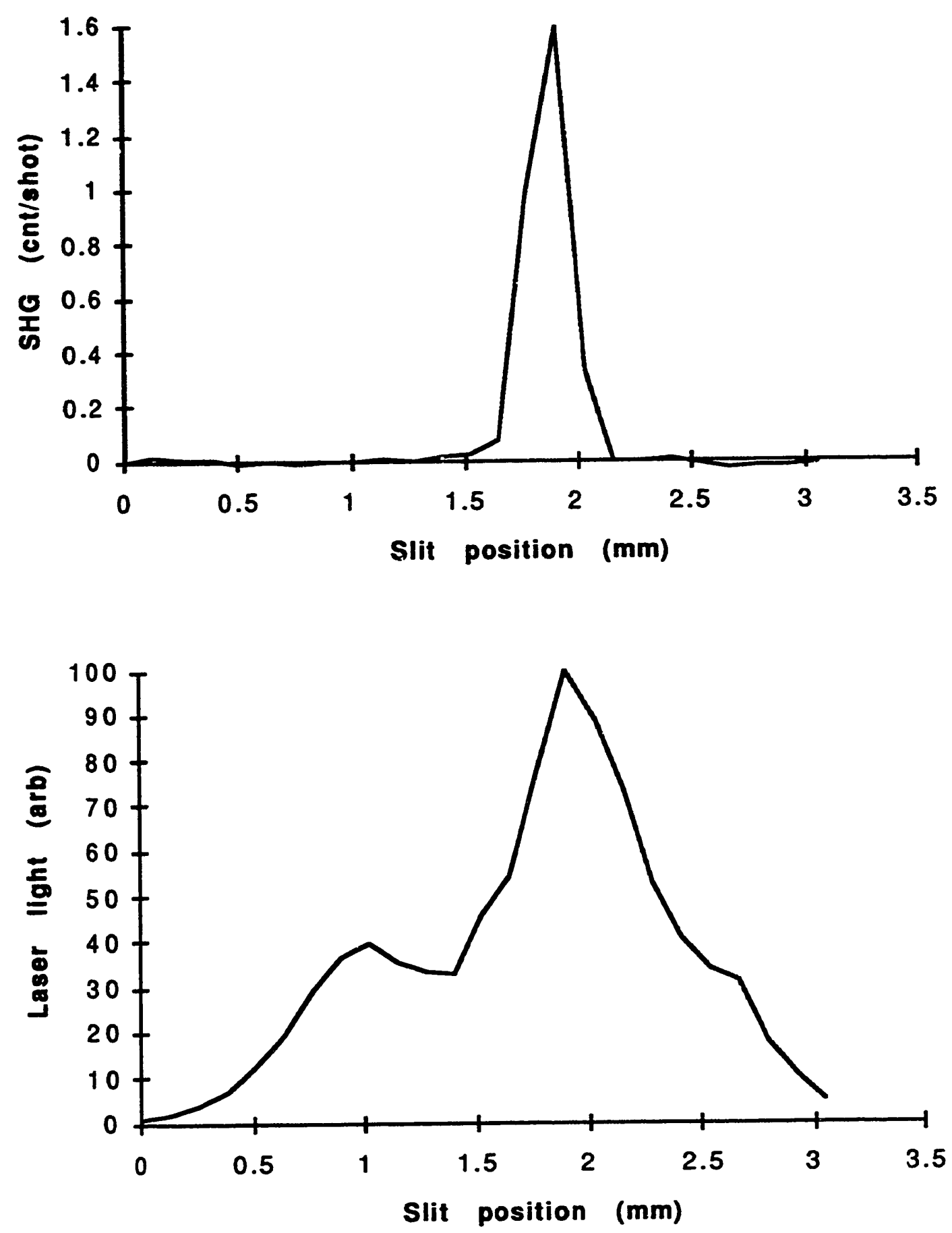

Figure 13 - Horizontal scans of a vertical slit across the SHG (top) and laser (bottom) output from the oven. The slit was $25 \mathrm{~cm}$ from the oven center, and the laser was unfocused going into the oven. 

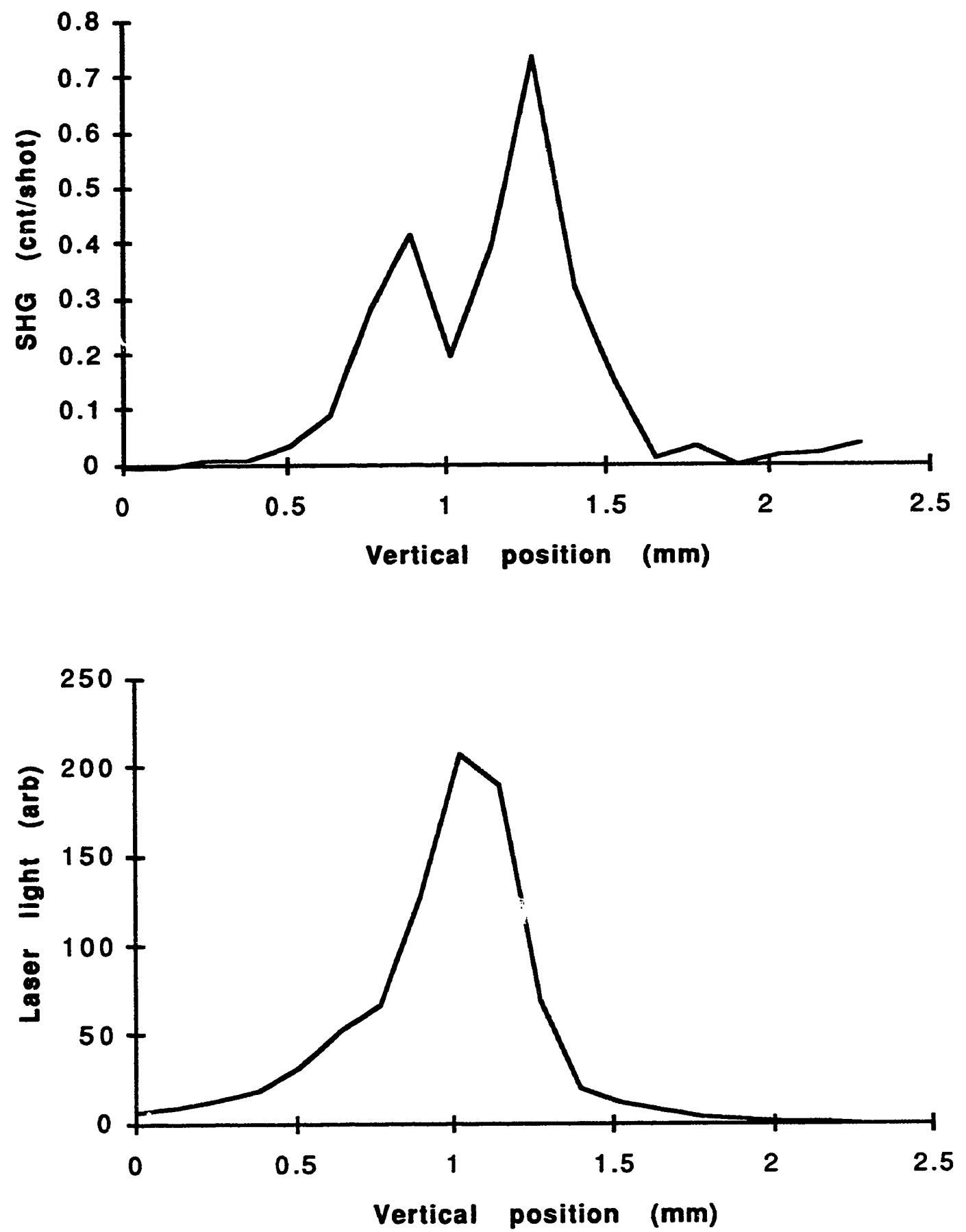

Figure 14 - Vertical scans of a horizontal slit, complementary to Figure 13.

The double-hump in the top figure (which was seen in all intensity bins; only the top bin is shown) shows that the SHG output is in two lobes. above and below the laser beam. 

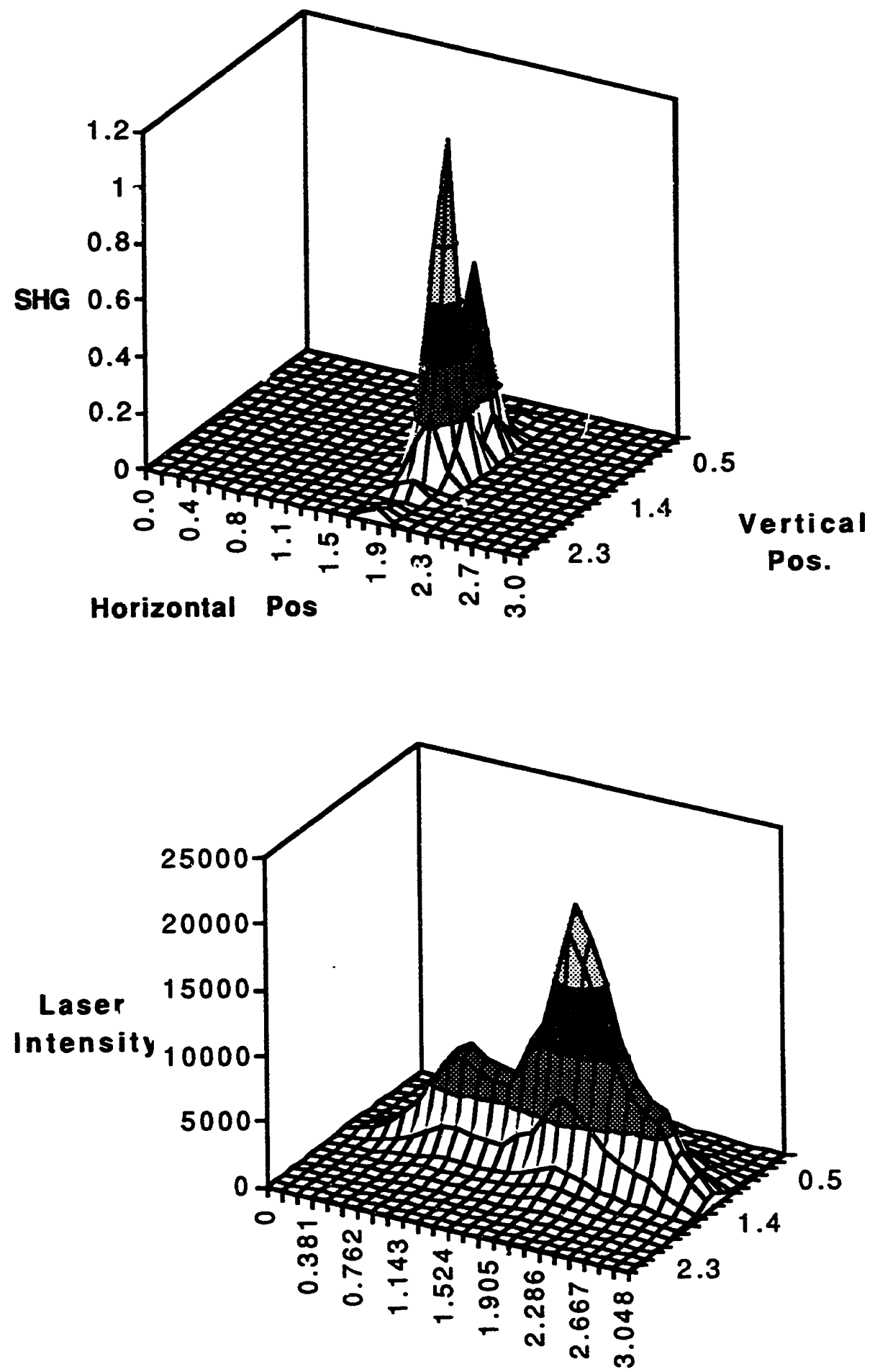

Figure 15 - Combined data from Figures 13 and 14, showing the narrow SHG output in two lobes (top) on the steepest gradient of the laser input (bottom). 


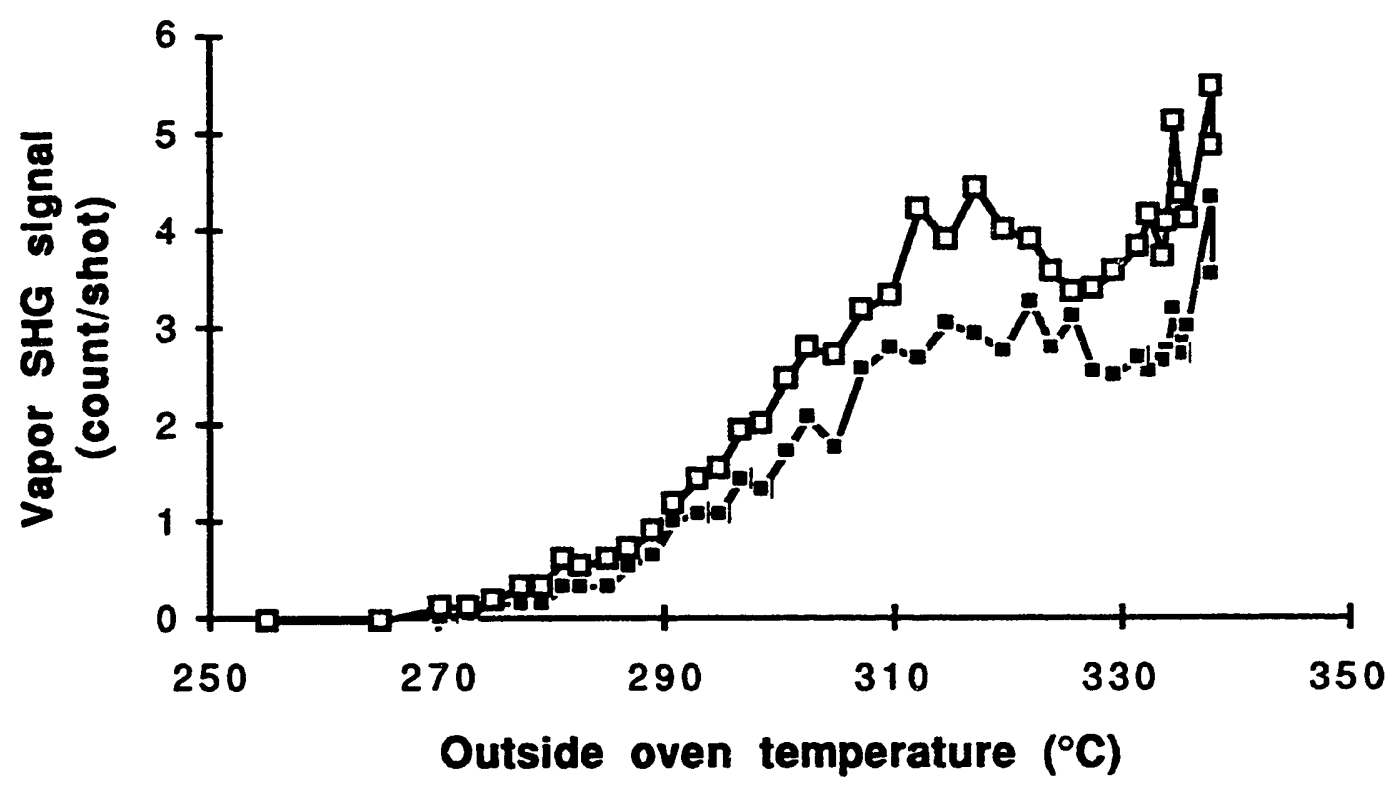

Figure 16 - Phase matching peak seen as a function of oven temperature. The different curves are for different laser beam energies. 


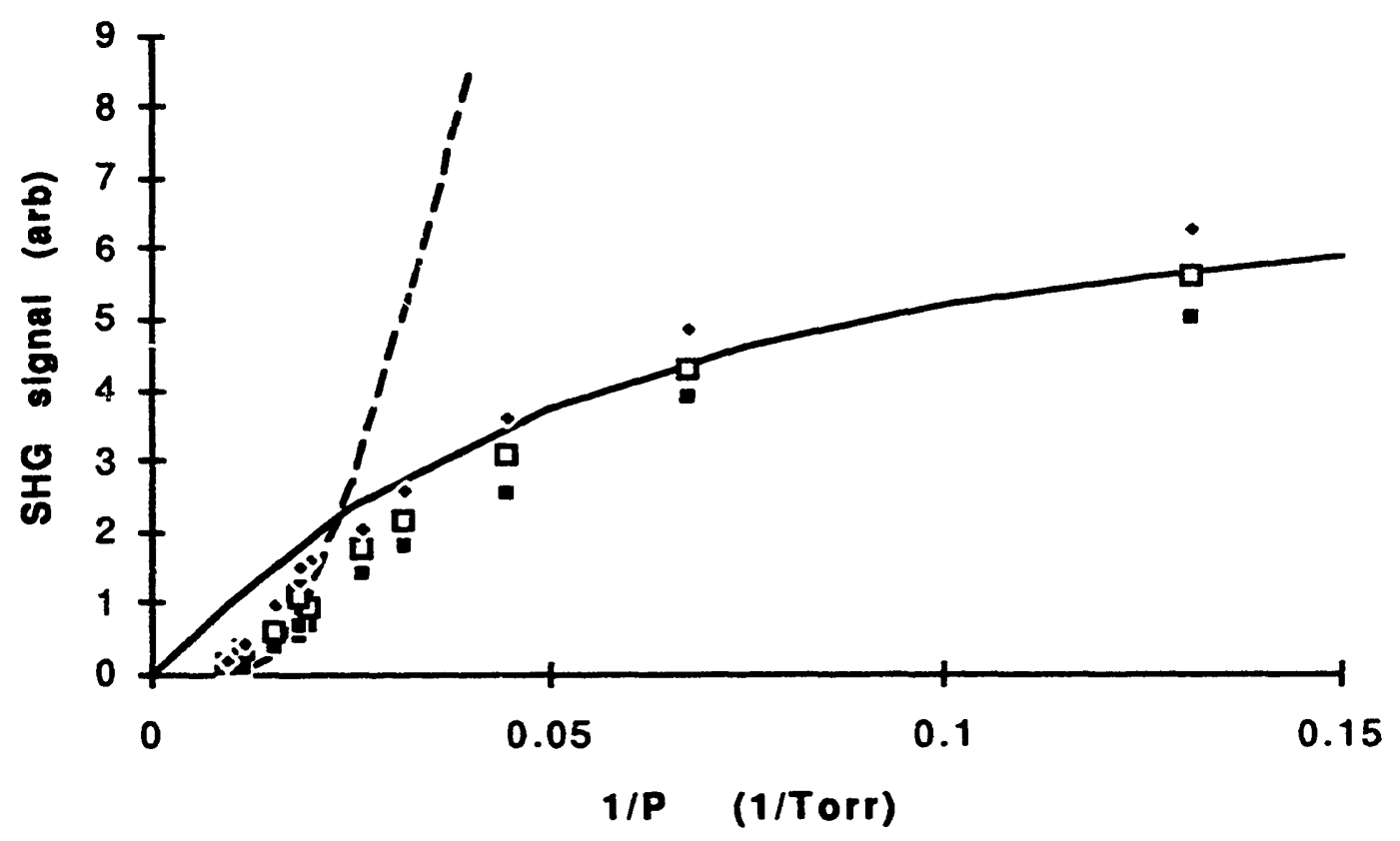

Figure 17 - Signal dependence on $\mathrm{Ar}$ pressure, shown versus 1/P to compare with the models. Constant symmetry-breaking predicts the solid line, which is proportional to $1 / \mathrm{P}$ at high pressures. If the symmetry changes with time, as the ionization model predicts (dotted line), then the signal is proportional to a higher power of $1 / P$. If the field saturates in time, (not accounted for in the dotted line), then the signal should curve over to the constant symmetry-breaking case. 


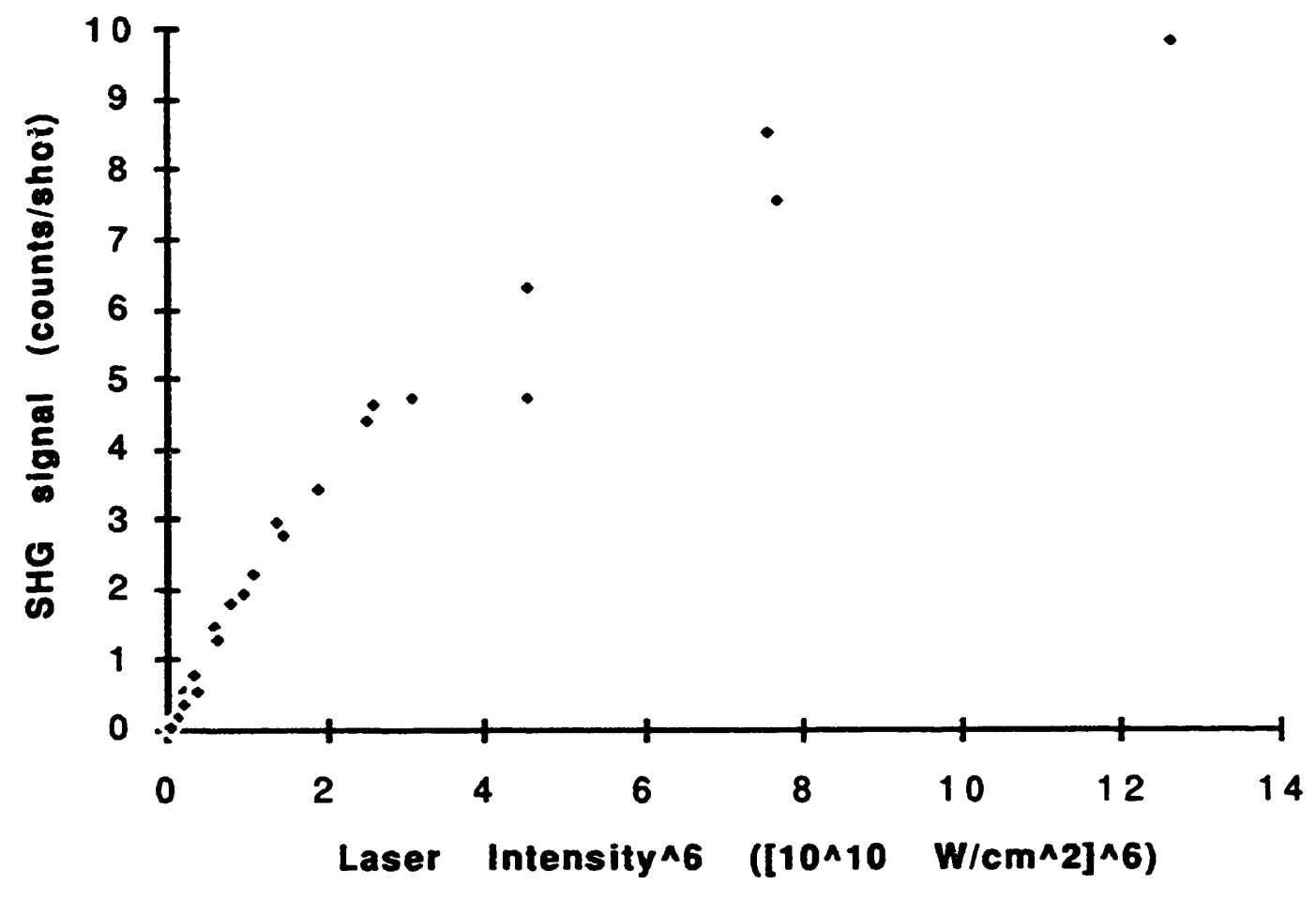

Figure 18 - Intensity dependence of vapor SHG, plotted vs. the sixth power of the laser intensity to demonstrate the strong dependence. Because it curves over, the I dependence weakens at higher intensities. Note that 1

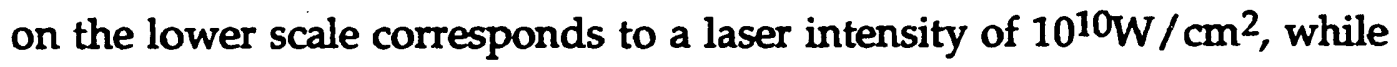
10 corresponds to $1.5 \times 10^{10} \mathrm{~W} / \mathrm{cm}^{2}$. 


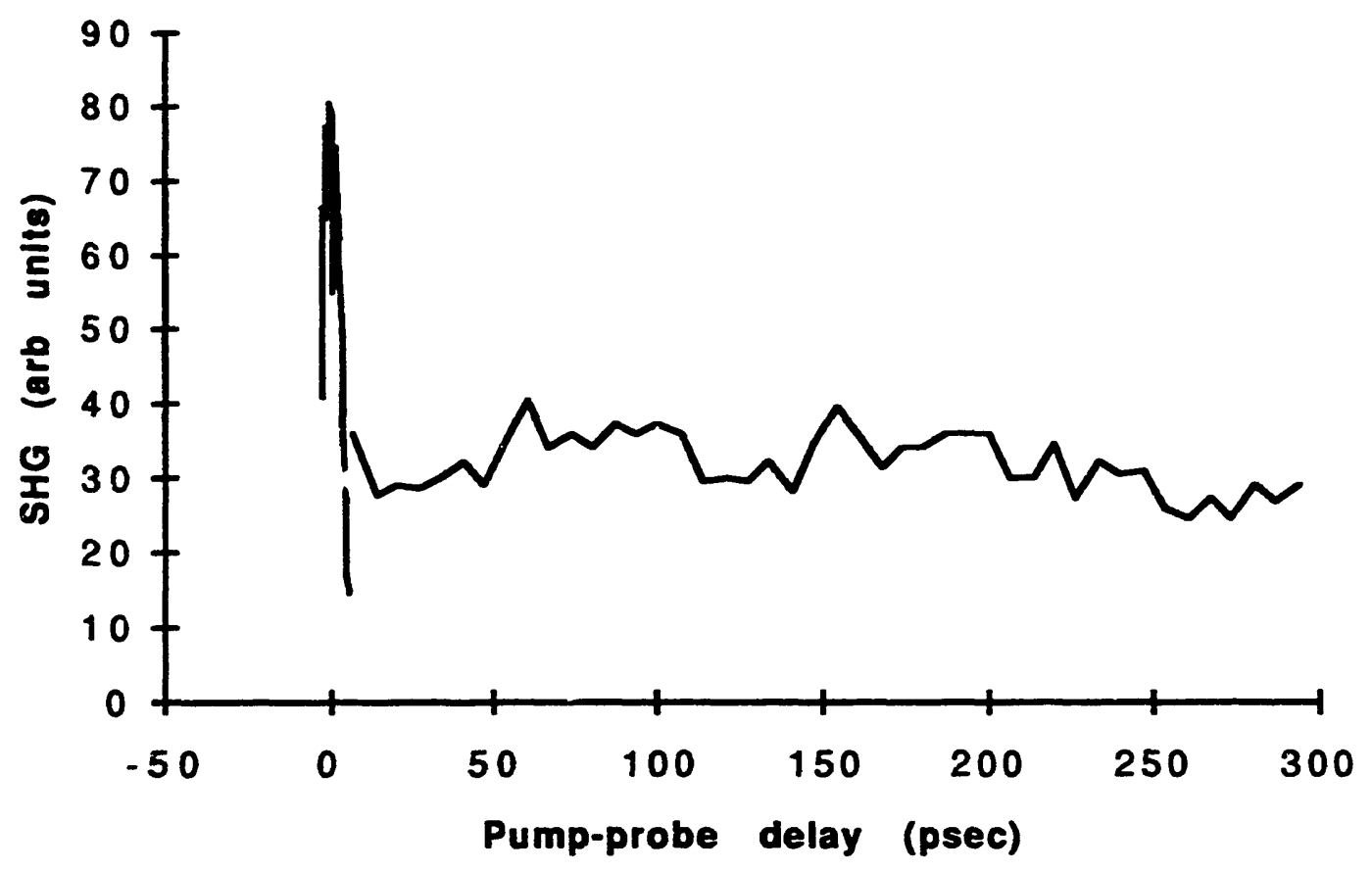

Figure 19 - Delay dependence curves in low-pressure potassium vapor. The autocorrelation peak is very narrow on this time scale, which is primarily meant to show the constant signal with long delays. 


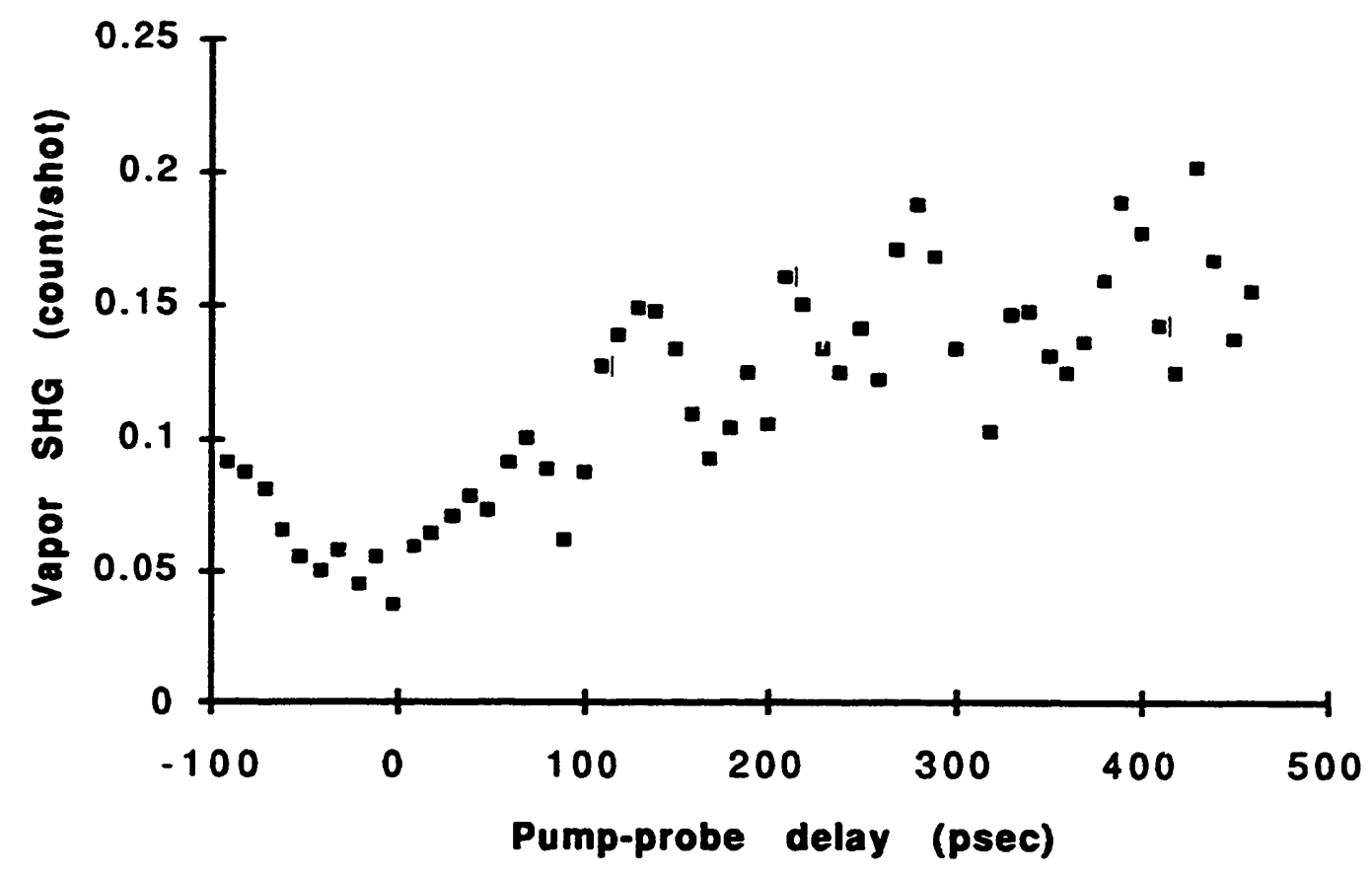

Figure 20 - Delay dependence of vapor SHG with 30 Torr Ar pressure. Signal rises with delay times up to $100 \mathrm{psec}$, where it appears to saturate. The scan is not fine enough to discern the autocorrelation peak near $\tau=0$. 


\section{Appendix: Uncertainty in Light Measurement}

Many experiments in this thesis use sensitive photon detectors or measure low levels of light. It is important to know how accurate the measurements are and how to make them better. Furthermore, fluctuations in the laser intensity should be taken into account by the use of a reference arm. This appendix discusses the issues involved in the detection and normalization of pulsed light sources.

\section{Ways to measure light}

When a photon strikes the photocathode of a photomultiplier tube, it releases a single electron with a certain quantum efficiency, typically $25 \%$ or less. This electron is accelerated toward the first of a series of dynodes, which it strikes, releasing more electrons. These new electrons are then accelerated toward the next dynode for further amplification. Ten to fifteen stages result in a net amplification of $10^{6}$ to $10^{7}$. The electrons form a current pulse at the anode. How this pulse is measured depends on the signal strength. Low signal levels are best measured by counting the proportion of shots which produce a current pulse, which is called photon counting. High signal levels are best measured by summing the current of all the pulses that are measured, which is called integration.

\section{A. Photon counting}

Photon counting uses the fact that the photon arrival is a Poisson process: photons arrive completely independent of each other. Strictly 
speaking, each arrival is binomial, but since they are independent of each other, the Poisson approximation is exact.

More precisely, the Poisson distribution is valid when the number of events is much smaller than the number of tests for an event. Then the probability of an event occurring during a single test must be very small. This can be applied to photons because the gate time $T$ can be divided up into many subintervals, with each interval testing for a photon arrival. If the arrivals are independent, an arrival of a photon in one subinterval will not affect the probability of arrival in any of other subinterval. Since the subintervals are arbitrarily small, the number of them is much larger than the number of photons that arrived during the whole interval $T$. Then the probability of an arrival in any one subinterval is very small, and the binomial probability is exactly approximated by the Poisson distribution. Thus the number of photons arriving during $T$ will always follow a Poisson distribution. The goal is to measure the intensity of light, $I$, in units of the average number of photons detected per interval. In a Poisson distribution, the probability of measuring $\mathbf{N}$ photons is:

$$
P(N, I)=\frac{I^{N}}{N !} e^{-I}
$$

Photon counting might be better described as "no-photon counting". During each interval, it is determined whether zero or more-than-zero photons arrived. Any information about how many photons arrived is thrown away. $\gamma$ is the ratio of more-than-zero photon intervals, $\Lambda$, to the total number of intervals measured, $M . \gamma$ is related to $I$ through the probability of measuring zero photons:

$$
\begin{gathered}
P(0, I)=e^{-I} \\
P(\text { Non-zero, } I)=1-P(0)=1-e^{-I}
\end{gathered}
$$


$\gamma$ is our measure of $P$ (Non-zero), so I is related to $\gamma$ by:

$$
I(\gamma)=-\ln (1-\gamma)
$$

For small $\gamma$, this equation can be approximated by:

$$
I(\gamma)=\gamma+\frac{\gamma^{2}}{2}+\frac{\gamma^{3}}{3}+\ldots
$$

This relation is within $10 \%$ of linear up to about $\gamma=0.2$.

\section{B. Integration}

Photon counting clearly breaks down for high enough intensities, where one or more photons are detected with each pulse of the laser. In the integration method the intensity of light is proportional to the sum of current pulses from the PMT. The intensity at which it becomes advantageous to integrate the signal instead of count it will be discussed below after a consideration of the uncertainties associated with each method of data acquisition.

At very high intensities, the PMT may saturate. Pulsed applications should use voltage divider networks with capacitors across the last few stages. Then saturation occurs (according to the Hamamatsu catalog) when

$$
C<100 \frac{\text { It }}{V} \text { (farads) }
$$

where $I t$ is the charge in the current pulse and $V$ is the voltage across the capacitor. For a total voltage of $1000 \mathrm{~V}$ across 10 stages, $\mathrm{V}=100$, and the capacitance in an E717-21 socket is $.02 \mu \mathrm{F}$, so the current pulse should be less than $.02 \mu$ Coulomb. This is equivalent to $3 \times 10^{4}$ detected photons with an amplification of $10^{7}$. Reference arms may reach this level, so care must be taken in using them. Surprisingly, with a 2 psec laser, we have also seen evidence of PMT saturation at count levels of just a few hundred photons/shot. With a strong reference arm signal, however, it is perhaps best to use a photodiode instead of a PMT. 


\section{IL. Uncertainty in light measurement}

\section{A. Photon counting}

To determine the uncertainty in a photon counting measurement, we must examine the measurement process. $\gamma$ is a measure over $M$ intervals, each of which gives one of two results, either zero photons or more-than-zero photon: Thus $\gamma$ will follow a binomial distribution, with the probabilities $p$ and $q$ defined in equations $\mathbf{2}$ and 3 as the probabilities of zero and non-zero photon results. Reif, in Statistical Physics, gives the dispersicn of a binomial distribution in equation 1.4.9. From that, the uncertainty in $\gamma$ can be written as a function of the intensity, $I$, and the number of measurements, $M$ :

$$
\Delta \gamma(I, M)=\sqrt{\frac{P(0, I) * P(\text { Non-zero, } I)}{M}}
$$

Since $\gamma$ is a measure of $\mathrm{P}($ Non-zero, $\mathrm{D})$ itself, the uncertainty in $\gamma$ is a function of $\gamma$.

$$
\Delta \gamma(\mathrm{I}, \mathrm{M})=\sqrt{\frac{(1-\gamma)^{*} \gamma}{\mathrm{M}}}
$$

The uncertainty in intensity measured is then $\mathrm{dI} / \mathrm{d} \gamma^{*} \Delta \gamma$.

$$
\Delta \mathrm{I}(\gamma, \mathrm{M})=\sqrt{\frac{\gamma}{(1-\gamma)^{*} \mathrm{M}}}
$$

and the relative uncertainty:

$$
\begin{aligned}
& \frac{\Delta I(\gamma, M)}{I}=\frac{-1}{\ln (1-\gamma)} \sqrt{\frac{\gamma}{(1-\gamma)^{*} M}} \\
& \approx-\frac{1}{\sqrt{M^{*} \gamma}} \text { for small } \gamma \\
& \approx \frac{1}{\sqrt{2^{*} M^{*}(1-\gamma)}} \text { for } \gamma \text { close to } 1
\end{aligned}
$$

Thus the relative uncertainty in intensity will be large when either $\gamma$ or $(1-\gamma)$ is small. It is graphed in figure 2. It has a minimum at a value of 
approximately 0.8 for $\gamma$. It is clear from the above equation that averaging over a greater number of laser shots (increasing $M$ ) always decreases the uncertainty in an intensity measurement, although such an increase eventually becomes prohibitive.

\section{B. Integration}

Integration sums over $M^{*} I$ photons, each of which experiences a gain $G$ that has an uncertainty $\Delta \mathrm{G}$. The total uncertainty in the intensity measurement is then the sum of two things: the uncertainty in number of photons measured and the uncertainty in the gain that each experienced:

$$
\begin{aligned}
\Delta I / I=\Delta\left(I^{*} M\right) & /\left(I^{*} M\right)=\frac{\sqrt{M^{*} I+M^{*} I^{*}(\Delta G / G)^{2}}}{M^{*} I} \\
& =\sqrt{\frac{1+(\Delta G / G)^{2}}{M^{*} I}}
\end{aligned}
$$

$M^{*} \mathrm{I}$ is the total number of photons detected, and each photon contributes a $\Delta G / G$ uncertainty to the total current pulse that is measured. One can immediately see that this is a greater uncertainty than that of photon counting for small signals. Since this is a monotonically decreasing function with $I$, it will at some point become more accurate than photon counting as a measure of intensity. For a typical $\Delta G / G$ value of 0.5 , this crossover occurs at a $\gamma$ value of 0.4 . Therefore, using the two methods in their respective regimes, one will always have greater accuracy with greater signal levels. To have the greatest dynamic range, one should use both techniques.

The above calculation ignores uncertainty in integration due to uncertainties in the background measurement. Estimating the RMS of the background signal at about $1 / 6$ the average photon signal, 100 pulses of averaging would generate 1.5 "photons-worth" of uncertainty, which will significantly increase the relative uncertainty at low signal levels $(<0.1$ 
photons/shot). Thus at low intensities, it becomes even more advantageous than it appears in Figure 3 to use photon counting instead of integration.

One last point for the curious: where does the uncertainty of gain in a PMT come from? At each stage, an accelerated electron releases other electrons in approximately Poisson process, so the end result is a Poisson process taken to a power of about 10.

If the total gain $G$ is a result of $m$ stages of smaller amplification, each with $g_{i}, i=1$ to $m$, then

$$
\begin{gathered}
G=\prod_{i=1}^{m} g_{i} \\
\Delta G=\sqrt{\sum_{i}\left(\frac{\partial G}{\partial g_{i}} \Delta g_{i}\right)^{2}} \\
\Delta g_{i}=\frac{\Delta g}{\sqrt{\prod_{j=1}^{i-1} n_{j}}}
\end{gathered}
$$

with

where $\Delta g$ is the uncertainty of the gain for a single electron and $\Delta g_{i}$ is the uncertainty of the average gain of electrons at stage $i$. If the electron gain is a Poisson process, then $\Delta g=\sqrt{g}$. For PMT's with several stages of equal gain $g$, the relative uncertainty is well-approximated by

$$
\Delta \mathrm{G} / \mathrm{G}=\frac{1}{\sqrt{\mathrm{g}-1}}
$$

For a 10-stage PMT with total ampli':cation of $10^{7}, g=5$ and $\Delta G / G=0.5$. It is also interesting to note that since most of the uncertainty comes from the first stage or two, increasing the gain there would decrease the relative uncertainty as a whole. 
The easiest way to calibrate the integrated signal values (i.e.. determine what $G$ is) is to measure a signal with $\gamma$ about 0.5 with both photon-counting and integration simultaneously (for instance, with CNTINTMODE in our FORTH software). This gives both a measured photon number (from the photon counting) and a corresponding integrated signal.

\section{Reference arms}

The uncertainties above are those inherent in the measurement process itself. Other uncertainties come into experiments in the form of an unstable laser and drift in alignment. A fixed, strong source of signal that will characterize the light going into the experiment can be used to account for some of the uncertainty. It is known as a reference arm.

There are two types of reference arms - linear and SHG. A linear arm measures a signal that is linear with the laser intensity, while an SHG arm uses a nonlinear crystal such as quartz and measures the SHG that results. For nonlinear experiments, an SHG reference arm better reflects the changes in pulse length, pulse shape, and transverse mode that affect the SHG efficiency. However, an SHG arm is more difficult to construct, align, and has a greater measurement uncertainty. Which type of arm is appropriate will depend on the experiment.

\section{A. Averaging over many pulses}

One common use of a reference arm is to average over as many shots as the signal. Since normalization is done only once every hundred or one thousand shots, fluctuations in the laser that take place on a shorter time scale cannot be compensated by this technique. A linear reference arm used on an SHG experiment should be squared with each shot before being 
summed because the mean of the squares is different from the square of the mean.

\section{B. Shot-to-shot normalization}

A technique which is occasionally mentioned, but rarely used, normalizes each shot separately. The difficulty is that the uncertainty in measurement of the signal arm in a single laser shot is very high, and is uncorrelated with the intensity of the laser. For instance, to be even $10 \%$ accurate, more than 100 photons must be detected in one shot. If the fluctuations in signal due to uncertainty are greater than those due to the laser fluctuations, then it is not clear how much information can be gained by trying to smooth out the laser fluctuations.

As an extreme example, consider the common case where one is photon counting the signal arm: one occasionally observes a photon in the signal arm, and one observes a fluctuating value in the reference arm. The reference arm would have to give a certain weight to the photons seen in the signal arm, as well as give a weight (or negative weight) to the times when there were no photons seen. This is difficult to express mathematically.

\section{Binning}

Probably the best way to account for large shot-to-shot fluctuations in the laser is to use several data storage areas, perhaps 10 . For each shot, the reference arm value is checked, and according to it, the signal arm data is processed into one of the storage areas. If the reference is strong, the data is kept in a higher bin, and if it is weak, it is kept in a lower bin. At the end of each point, the data in each bin can be weighted by the value of the reference arm for that bin. The uncertainty in single measurements is avoided because 
for each reference intensity, many laser shots are accumulated. I have successfully used this technique. It also provides an automatic measurement of the signal's dependence on laser intensity.

\section{Checking a reference arm}

To be effective, the uncertainty in the reference arm measurement must be less than the laser fluctuations. For an integrated signal, Equation (13) shows $\Delta I / I$ will be $10 \%$ if the total number of photons is about 150 . To achieve $1 \%$ accuracy in the pulse-to-pulse referenre arm measurement would require 15,000 photons. With care such signals can be generated in phasematched nonlinear crystals. A linear reference arm, on the other hand, can easily achieve such high photon numbers. Both are potentially useful for shot-to-shot normalization or the binning method described above.

To determine the accuracy of a reference arm, one constructs two such arms and compares the resulting signals. In the case of SHG arms, the second arm is typically in place of the sample arm. To compare shot-to-shot signals, the two measured values can be used as $x$ - and $y$-values for a point plotted on the screen, and the correlation between $x$ and $y$ can be calculated over many shots. It appears on the screen as an elliptical cloud of dots, or a sloping line of dots, or somewhere in between. The more correlation there is, the more closely the points adhere to the line.

Two SHG reference arms show good correlation when both use a phase-matched nonlinear crystal and a photodiode to measure the signal. Linear arms observing the attenuated laser beam typically have adequate signal levels. However, car must be taken in aligning the photodiode in either case. Either a lens or a diffuser should be used. Accurac es are typically better than $1 \%$. The "diffuser" can be fogged glass (cut from a microscope 
slide) inserted in a slot cia:? in a heavy cardboard tube. The tube is from a FAX paper roll, cut to about $5 \mathrm{~cm}$, and additional slots can accommodate color filters or neutral density filters.

For an averaged reference arm, one need simply measure the correlation of the averages. The measured accuracy is only meaningful when compared with the expected changes in laser intensity that are anticipated. 


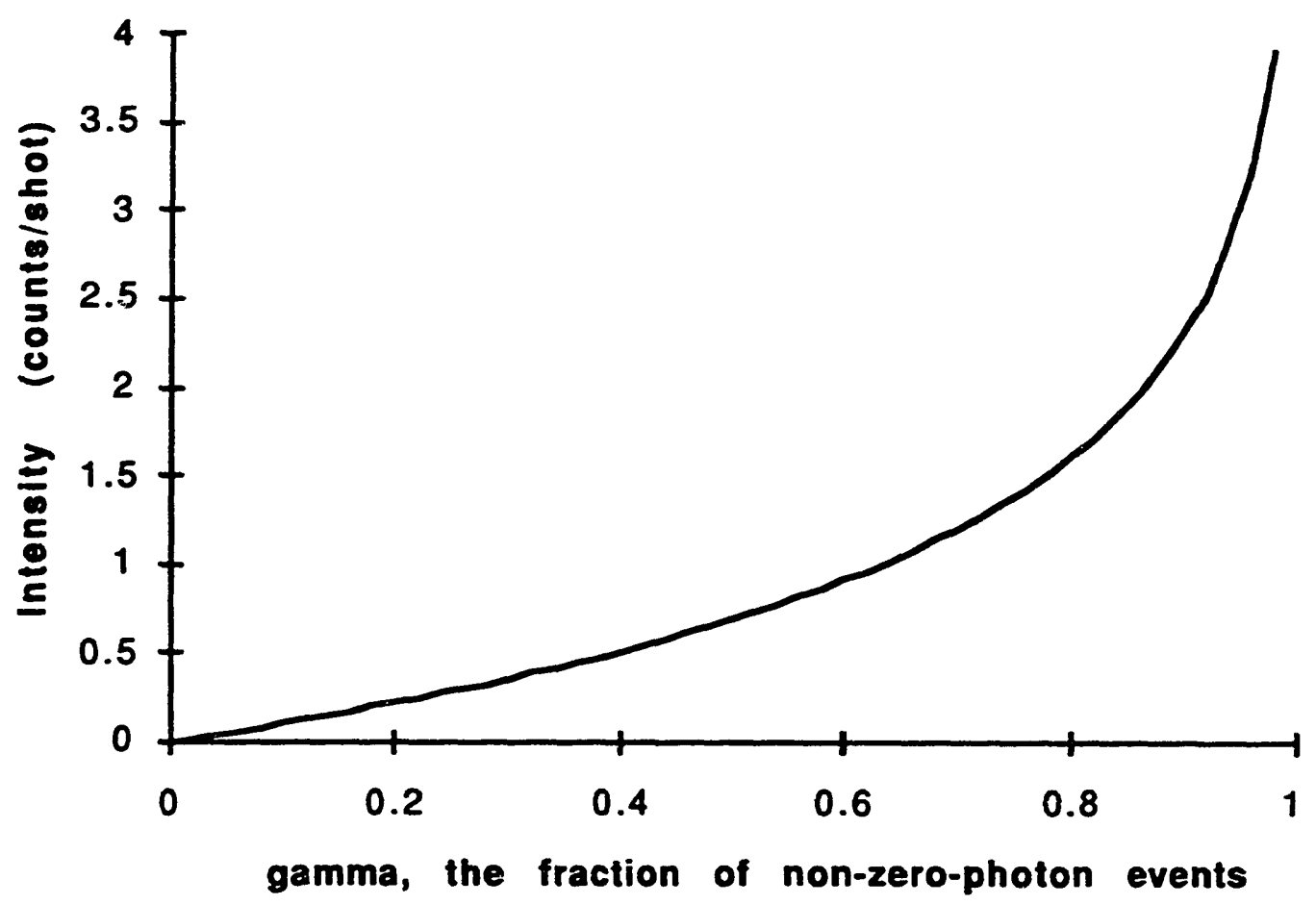

Figure 1: Intensity versus $\gamma$ for a photon-counting measurement, according to Equation (4). 


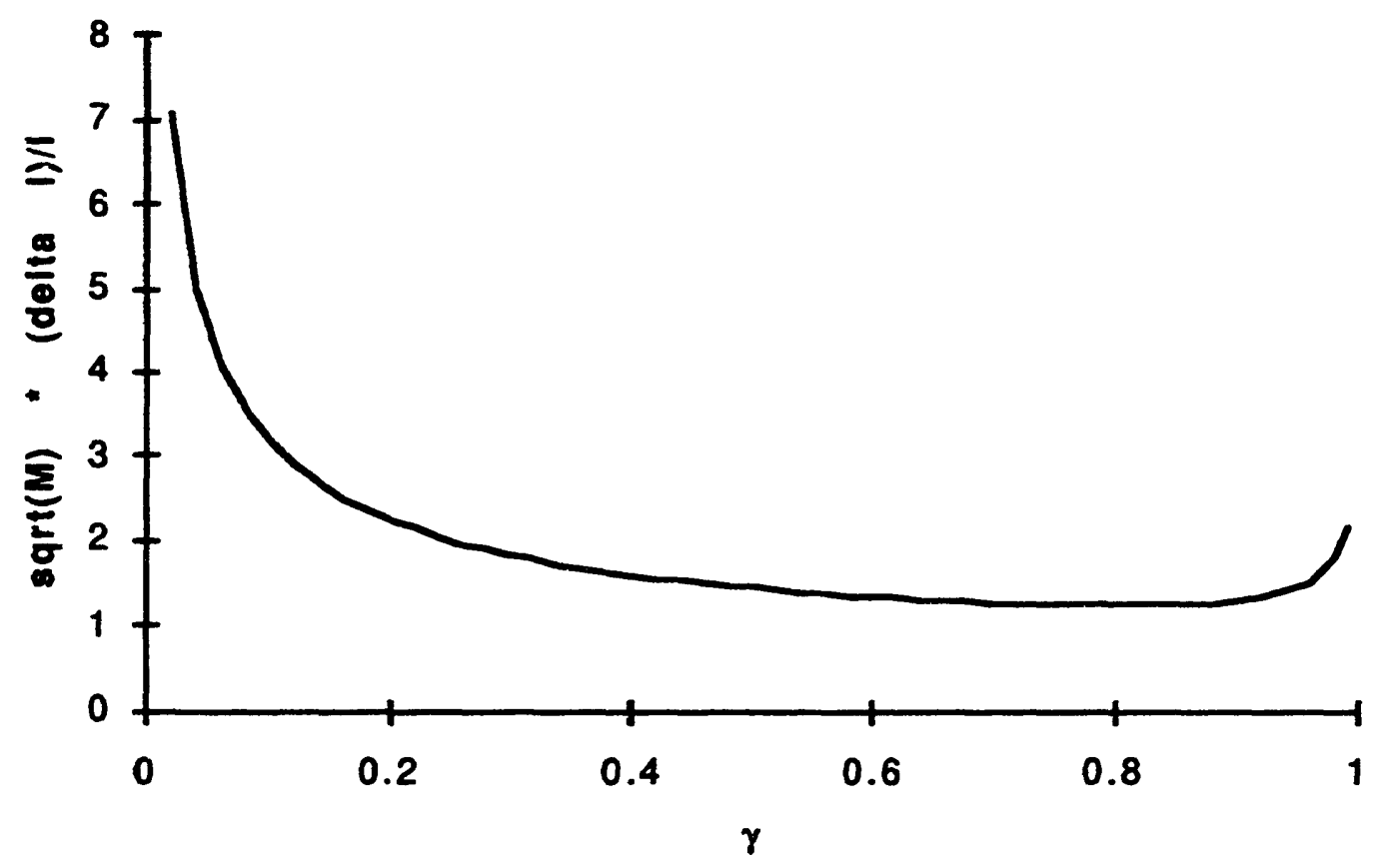

Figure 2: Relative uncertainty in intensity for a photon-counting measurement, where $M$ is the number of shots over which it is measured. For example, if $\gamma=0.2$, for which the graph reads 2 , then measuring for 100 shots will make the relative uncertainty $20 \%$. 


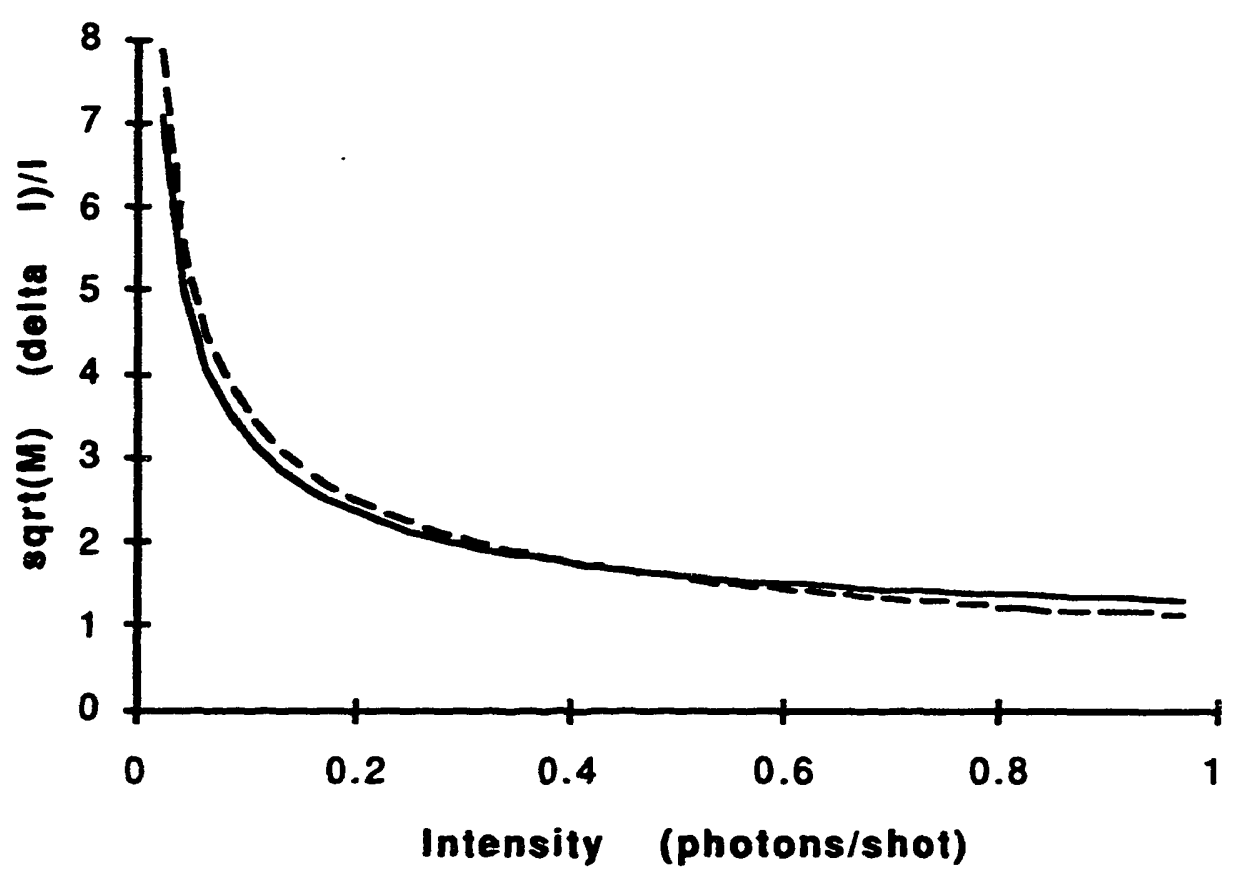

Figure 3: Relative uncertainty in intensity measurement for photon counting (solid line) and integration, assuming $\Delta \mathrm{G} / \mathrm{G}=0.5$ (dotted line). 

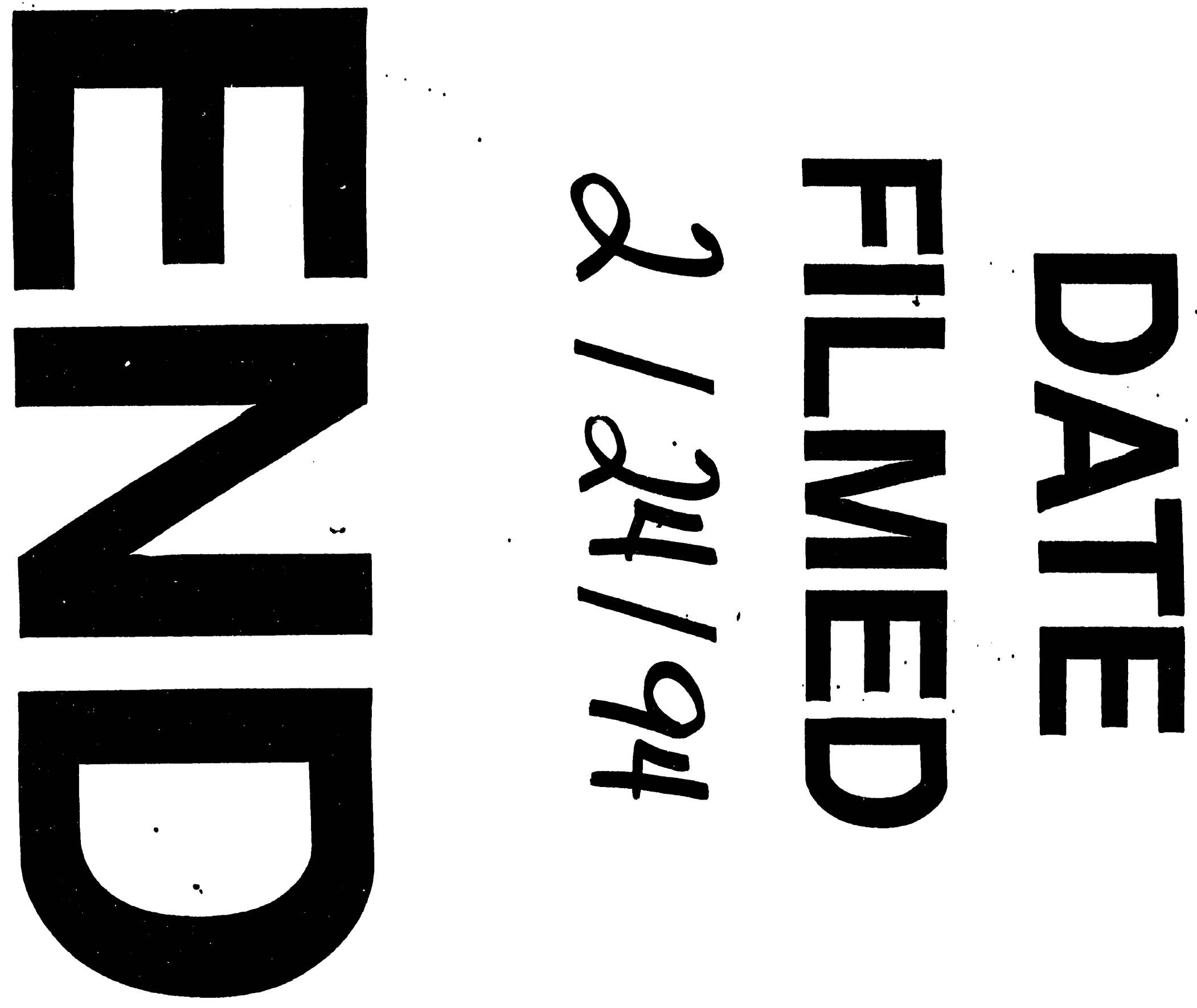